J. Appl. Numer. Optim. 3 (2021), No. 2, pp. 361-401

Available online at http://jano.biemdas.com

https://doi.org/10.23952/jano.3.2021.2.08

\title{
CONVERGENCE OF IMPLICIT RUNGE-KUTTA TIME DISCRETISATION METHODS FOR FUNDAMENTAL MODELS IN NONLINEAR ACOUSTICS
}

\author{
BARBARA KALTENBACHER ${ }^{1}$, MECHTHILD THALHAMMER $^{2, *}$ \\ ${ }^{1}$ Department of Mathematics, Alpen-Adria-Universität Klagenfurt, 9020 Klagenfurt, Austria \\ ${ }^{2}$ Department of Mathematics, Leopold-Franzens-Universität Innsbruck, 6020 Innsbruck, Austria
}

\begin{abstract}
For the first time, a class of implicit Runge-Kutta time discretisation methods is studied for nonlinear damped wave equations arising in nonlinear acoustics. The analysis in particular applies to the Westervelt, Jordan-Moore-Gibson-Thompson, and Blackstock-Crighton-Brunnhuber-JordanKuznetsov equations. Under appropriate regularity, consistency, and smallness requirements on the time-continuous solutions, global error bounds are obtained from energy estimates for the time-discrete solutions. Existence and uniqueness of time-discrete solutions as well as their convergence are proven under weaker conditions on the initial data, based on energy estimates that are established in a continuous setting and then transferred to the time discretisations.
\end{abstract}

Keywords. High-intensity ultrasonics; Implicit Runge-Kutta methods; Nonlinear damped wave equations; Nonlinear acoustics; Time discretisation.

\section{INTRODUCTION}

In this contribution, we introduce stiffly accurate Runge-Kutta methods for the time integration of nonlinear damped wave equations representing classical and advanced models of nonlinear acoustics. This class of implicit one-step methods includes as simplest instance the backward Euler method, and it is seen to be particularly suited for a study based on variational formulations and energy estimates.

Proceeding former work on stiffly accurate Runge-Kutta methods for nonlinear evolutionary problems, see [1] and [2], we establish existence, boundedness, and convergence of the timediscrete solutions as well as global error bounds under regularity assumptions on the solutions, that identify the stage order as decisive quantity.

The present manuscript is organised as follows. In Section 2, we specify the considered fundamental models, the Westervelt equation, the Jordan-Moore-Gibson-Thompson equation, and the Blackstock-Crighton-Brunnhuber-Jordan-Kuznetsov equation. In Section 3, we state the general format of stiffly accurate Runge-Kutta methods and a basic condition on the coefficients that is essential in view of our variational approach. Section 4 is devoted to the derivation of global error bounds by means of suitable Taylor series expansions and higher-order energy

\footnotetext{
${ }^{*}$ Corresponding author.

E-mail addresses: barbara.kaltenbacher@aau.at (B. Kaltenbacher), mechthild.thalhammer@uibk.ac.at (M. Thalhammer).

Received November 23, 2020; Accepted February 23, 2021.
}

(C)2021 Journal of Applied and Numerical Optimization 
estimates. Sections 5 and 6 provide energy estimates for the time-continuous and time-discrete systems in a more general framework. The latter also enables to establish existence and uniqueness of time-discrete solutions as well as their convergence as the time stepsize tends to zero.

We note that our study includes variable time stepsizes under appropriate smallness requirements, which are employed in the derivation of energy estimates, see Section 4. Merely to reduce the amount of technicalities, we assume the time grid to be uniform in Section 6 .

\section{Fundamental Models}

The field of nonlinear acoustics is concerned with the propagation of sound waves in thermoviscous fluids. This includes a wide range of applications, in particular in high-intensity ultrasonics $[3,4,5]$. In this context, the underlying mathematical models are generally based on nonlinear partial differential equations, and reliable as well as efficient numerical solvers are essential tools in view of arising design and monitoring tasks. As relevant application, we mention the shape design of an acoustic lens for focusing high-intensity ultrasound in medical treatment such as lithotripsy.

In the following, we state the fundamental models investigated in this work and introduce compact reformulations as first-order evolutionary systems. We begin with the Westervelt (W) equation, a well-known and oftentimes examined strongly damped wave equation that takes into account nonlinear effects but neglects thermal losses. A generalisation that additionally comprises a quadratic velocity term to reflect a local nonlinearity is the Kuznetsov equation. There exist numerous extensions of these two classical models avoiding the infinite signal speed paradox or incorporating thermal effects, respectively. We focus on the JordanMoore-Gibson-Thompson (JMGT) equation and the Blackstock-Crighton-Brunnhuber-JordanKuznetsov (BCBJK) equation. Detailed information on the underlying physics is found in the by now classical references $[6,7,8,9,10,11,12,13,14,15]$. For a review of recent results on the analyis of nonlinear damped wave equations and further references to significant contributions, we refer to [16].

In essence, the employed setting and notation accord to our former work [17]. Throughout, we consider a bounded spatial domain $\Omega \subset \mathbb{R}^{3}$ with sufficiently regular boundary and a finite time interval $[0, T]$. Regarding convenient reformulations as evolution equations, we set

$$
\mathscr{A}=-\Delta \text {, }
$$

where $\Delta$ denotes the Laplacian with respect to the spatial variables. For simplicity, we restrict ourselves to homogeneous Dirichlet boundary conditions. We use standard notation for Lebesgue and Sobolev spaces.

Westervelt equation. The Westervelt equation [15] can be cast into the form

$$
\left\{\begin{array}{c}
\partial_{t t} p(x, t)-b \Delta \partial_{t} p(x, t)-c^{2} \Delta p(x, t) \\
=\frac{\beta_{a}}{\rho c^{2}} \partial_{t t}(p(x, t))^{2}, \quad(x, t) \in \Omega \times(0, T),
\end{array}\right.
$$

with $p: \Omega \times[0, T] \rightarrow \mathbb{R}$ the acoustic pressure, $b>0$ the diffusivity of sound, $c>0$ the speed of sound, $\beta_{a}>0$ the parameter of nonlinearity, and $\rho>0$ the mean mass density. Performing differentiation on the right hand side, it is seen that degeneracy is exhibited when the multiplier of the second time derivative vanishes or becomes negative

$$
\left(1-\frac{2 \beta_{a}}{\rho c^{2}} p(x, t)\right) \partial_{t t} p(x, t)-b \Delta \partial_{t} p(x, t)-c^{2} \Delta p(x, t)=\frac{2 \beta_{a}}{\rho c^{2}}\left(\partial_{t} p(x, t)\right)^{2} .
$$


Under certain regularity, consistency, and smallness requirements on the initial data, using a suitable linearisation of the equation that defines an analytic semigroup and maximal parabolic regularity in appropriate function spaces, results on well-posedness and asymptotic behavior are deduced in $[18,19]$. In this situation, it is justified to investigate the following reformulation as abstract evolution equation

$$
\begin{aligned}
& \left\{\begin{array}{c}
u^{\prime \prime}(t)+\mathrm{b}\left(u(t), u^{\prime}(t)\right) \mathscr{A} u^{\prime}(t)+\mathrm{c}_{1}\left(u(t), u^{\prime}(t)\right) \mathscr{A} u(t) \\
=\mathscr{B}\left(u(t), u^{\prime}(t)\right)\left[u(t), u^{\prime}(t)\right], \quad t \in(0, T),
\end{array}\right. \\
& \alpha(v)=1-\frac{2 \beta_{a}}{\rho c^{2}} v, \quad \widetilde{\alpha}(v)=(\alpha(v))^{-1}, \\
& \mathrm{~b}\left(v_{0}, v_{1}\right)=b \widetilde{\alpha}\left(v_{0}\right), \quad \mathrm{c}_{1}\left(v_{0}, v_{1}\right)=c^{2} \widetilde{\alpha}\left(v_{0}\right), \quad \mathscr{B}\left(v_{0}, v_{1}\right)\left[u_{0}, u_{1}\right]=\frac{2 \beta_{a}}{\rho c^{2}} \widetilde{\alpha}\left(v_{0}\right) v_{1} u_{1} .
\end{aligned}
$$

With regard to the introduction and global error analysis of the implicit Euler method and, more generally, stiffly accurate Runge-Kutta methods, it is helpful to rewrite the Westervelt equation as first-order evolutionary system

$$
\begin{gathered}
\mathbf{u}^{\prime}(t)+\mathbf{A}(\mathbf{u}(t)) \mathbf{u}(t)=\mathbf{B}(\mathbf{u}(t))[\mathbf{u}(t)], \quad t \in(0, T) \\
\mathbf{A}(\mathbf{u}(t))=\left(\begin{array}{cc}
0 & -I \\
\mathrm{c}_{1}(\mathbf{u}(t)) \mathscr{A} & \mathrm{b}(\mathbf{u}(t)) \mathscr{A}
\end{array}\right), \quad \mathbf{B}(\mathbf{u}(t))[\mathbf{u}(t)]=\left(\begin{array}{cc}
0 \\
\mathscr{B}(\mathbf{u}(t))[\mathbf{u}(t)]
\end{array}\right),
\end{gathered}
$$

where $\mathbf{u}=\left(u_{0}, u_{1}\right)^{T}$ represents the solution $u$ and its time derivative $u^{\prime}$.

Jordan-Moore-Gibson-Thompson equation. Replacing in the derivation of the Westervelt equation the Fourier law for the heat flux by a Maxwell-Cattaneo law avoids the infinite signal speed paradox [20] and leads to the Jordan-Moore-Gibson-Thompson equation

$$
\left\{\begin{array}{c}
T_{r e l} \partial_{t t t} p(x, t)+\partial_{t t} p(x, t)-b \Delta \partial_{t} p(x, t)-c^{2} \Delta p(x, t) \\
=\frac{\beta_{a}}{\rho c^{2}} \partial_{t t}(p(x, t))^{2}, \quad(x, t) \in \Omega \times(0, T),
\end{array}\right.
$$

with $T_{\text {rel }}>0$ denoting the relaxation time. Compared to (2.1), the appearance of the third-order time derivative considerably changes the character of the equation. It prevents analyticity of the semigroup defined by the linearised equation. For parameter ranges $b<T_{\text {rel }} c^{2}$ even continuity of the semigroup is lost, and the problem is ill-posed. As significant contributions in this context, we mention [21, 22, 23, 24, 25, 26, 27]. We study the reformulation

$$
\begin{gathered}
\left\{\begin{array}{c}
u^{\prime \prime \prime}(t)+\mathrm{b}\left(u(t), u^{\prime}(t), u^{\prime \prime}(t)\right) u^{\prime \prime}(t)+\mathrm{c}_{1}\left(u(t), u^{\prime}(t), u^{\prime \prime}(t)\right) \mathscr{A} u^{\prime}(t) \\
=\mathscr{B}\left(u(t), u^{\prime}(t), u^{\prime \prime}(t)\right)\left[u(t), u^{\prime}(t), u^{\prime \prime}(t)\right], \quad t \in(0, T),
\end{array}\right. \\
\alpha(v)=1-\frac{2 \beta_{a}}{\rho c^{2}} v, \quad \mathrm{~b}\left(v_{0}, v_{1}, v_{2}\right)=\frac{1}{T_{r e l}} \alpha\left(v_{0}\right), \quad \mathrm{c}_{1}\left(v_{0}, v_{1}, v_{2}\right)=\frac{b}{T_{\text {rel }}}, \\
\mathscr{B}\left(v_{0}, v_{1}, v_{2}\right)\left[u_{0}, u_{1}, u_{2}\right]=-\frac{c^{2}}{T_{r e l}} \mathscr{A} u_{0}+\frac{2 \beta_{a}}{\rho c^{2} T_{\text {rel }}} v_{1} u_{1} .
\end{gathered}
$$

The associated first-order evolutionary system is given by

$$
\begin{gathered}
\mathbf{u}^{\prime}(t)+\mathbf{A}(\mathbf{u}(t)) \mathbf{u}(t)=\mathbf{B}(\mathbf{u}(t))[\mathbf{u}(t)], \quad t \in(0, T), \\
\mathbf{A}(\mathbf{u}(t))=\left(\begin{array}{ccc}
0 & -I & 0 \\
0 & 0 & -I \\
0 & \mathrm{c}_{1}(\mathbf{u}(t)) \mathscr{A} & \mathrm{b}(\mathbf{u}(t))
\end{array}\right), \quad \mathbf{B}(\mathbf{u}(t))[\mathbf{u}(t)]=\left(\begin{array}{c}
0 \\
0 \\
\mathscr{B}(\mathbf{u}(t))[\mathbf{u}(t)]
\end{array}\right),
\end{gathered}
$$

with $\mathbf{u}=\left(u_{0}, u_{1}, u_{2}\right)$ comprising the solution as well as the first and second time derivatives. 
Blackstock-Crighton-Brunnhuber-Jordan-Kuznetsov equation. The Blackstock-CrightonBrunnhuber-Jordan-Kuznetsov equation [6, 28, 29] is an extension of the Westervelt and Kuznetsov equations that takes into account thermal effects. Accordingly to [17], we consider the formulation

$$
\left\{\begin{array}{c}
\partial_{t t t} \psi(x, t)-\beta_{1} \Delta \partial_{t t} \psi(x, t)+\beta_{2} \Delta^{2} \partial_{t} \psi(x, t)-\beta_{3} \Delta \partial_{t} \psi(x, t)+\beta_{4} \Delta^{2} \psi(x, t) \\
=-\partial_{t t}\left(\frac{1}{2} \beta_{5}\left(\partial_{t} \psi(x, t)\right)^{2}+\beta_{6}|\nabla \psi(x, t)|^{2}\right), \quad(x, t) \in \Omega \times(0, T)
\end{array}\right.
$$

with acoustic velocity potential $\psi: \Omega \times[0, T] \rightarrow \mathbb{R}$ and certain constants $\beta_{i}>0$ for $i \in\{1, \ldots, 6\}$. In $[17,30]$, well-posedness is studied and a rigorous justification of the Westervelt and Kuznetsov equations as limiting models is given. We employ the reformulation

$$
\begin{gathered}
\left\{\begin{array}{c}
u^{\prime \prime \prime}(t)+\mathrm{b}\left(u(t), u^{\prime}(t), u^{\prime \prime}(t)\right) \mathscr{A} u^{\prime \prime}(t) \\
+\mathrm{c}_{1}\left(u(t), u^{\prime}(t), u^{\prime \prime}(t)\right) \mathscr{A} u^{\prime}(t)+\mathrm{c}_{2}\left(u(t), u^{\prime}(t), u^{\prime \prime}(t)\right) \mathscr{A}^{2} u^{\prime}(t) \\
=\mathscr{B}\left(u(t), u^{\prime}(t), u^{\prime \prime}(t)\right)\left[u(t), u^{\prime}(t), u^{\prime \prime}(t)\right], \quad t \in(0, T), \\
\alpha(v)=1+\beta_{5} v, \quad \widetilde{\alpha}(v)=(\alpha(v))^{-1}, \\
\mathrm{~b}\left(v_{0}, v_{1}, v_{2}\right)=\beta_{1} \widetilde{\alpha}\left(v_{1}\right), \quad \mathrm{c}_{1}\left(v_{0}, v_{1}, v_{2}\right)=\beta_{3} \widetilde{\alpha}\left(v_{1}\right), \quad \mathrm{c}_{2}\left(v_{0}, v_{1}, v_{2}\right)=\beta_{2} \widetilde{\alpha}\left(v_{1}\right), \\
\mathscr{B}\left(v_{0}, v_{1}, v_{2}\right)\left[u_{0}, u_{1}, u_{2}\right]=-\widetilde{\alpha}\left(v_{1}\right)\left(\beta_{4} \mathscr{A}^{2} u_{0}+\beta_{5} v_{2} u_{2}+2 \beta_{6} \nabla v_{1} \cdot \nabla u_{1}\right. \\
\left.+2 \beta_{6} \nabla v_{0} \cdot \nabla u_{2}\right) .
\end{array}\right.
\end{gathered}
$$

The corresponding first-order evolutionary system reads as

$$
\begin{aligned}
\mathbf{u}^{\prime}(t)+\mathbf{A}(\mathbf{u}(t)) \mathbf{u}(t)=\mathbf{B}(\mathbf{u}(t))[\mathbf{u}(t)], & t \in(0, T) \\
\mathbf{A}(\mathbf{u}(t))= & \left(\begin{array}{ccc}
0 & -I & 0 \\
0 & 0 & -I \\
0 & \mathrm{c}_{1}(\mathbf{u}(t)) \mathscr{A}+\mathrm{c}_{2}(\mathbf{u}(t)) \mathscr{A}^{2} & \mathrm{~b}(\mathbf{u}(t)) \mathscr{A}
\end{array}\right) \\
& \mathbf{B}(\mathbf{u}(t))[\mathbf{u}(t)]=\left(\begin{array}{c}
0 \\
0 \\
\mathscr{B}(\mathbf{u}(t))[\mathbf{u}(t)]
\end{array}\right)
\end{aligned}
$$

where $\mathbf{u}=\left(u_{0}, u_{1}, u_{2}\right)$ again represents the solution as well as the first and second time derivatives.

Compact formulation. Setting

$$
\begin{aligned}
& \text { (W) } m=1: k_{1}=1, r=1: \ell_{1}=1 \text {, } \\
& \text { (JMGT) } m=2: k_{2}=0, r=1: \ell_{1}=1 \text {, } \\
& \text { (BCBJK) } m=2: k_{2}=1, r=2: \ell_{1}=1, \ell_{2}=2 \text {, }
\end{aligned}
$$

we can cast the Westervelt equation (2.1), the Jordan-Moore-Gibson-Thompson equation (2.2), and the Blackstock-Crighton-Brunnhuber-Jordan-Kuznetsov equation (2.3) into the form of a 
higher-order evolution equation

$$
\left\{\begin{aligned}
& u^{(m+1)}(t)+\mathrm{b}\left(u(t), u^{\prime}(t), \ldots, u^{(m)}(t)\right) \mathscr{A}^{k_{m}} u^{(m)}(t) \\
&+\sum_{i=1}^{r} \mathrm{c}_{i}\left(u(t), u^{\prime}(t), \ldots, u^{(m)}(t)\right) \mathscr{A}^{\ell_{i}} u^{(m-1)}(t) \\
&=\mathscr{B}\left(u(t), u^{\prime}(t), \ldots, u^{(m)}(t)\right)\left[u(t), u^{\prime}(t), \ldots, u^{(m)}(t)\right], \quad t \in(0, T),
\end{aligned}\right.
$$

with non-negative integers $k_{m} \geq 0$ and $0 \leq \ell_{1}<\ell_{2}<\cdots<\ell_{r}$. Accordingly, the associated first-order evolutionary system is given by

$$
\begin{gathered}
\mathbf{u}^{\prime}(t)=\mathbf{F}(\mathbf{u}(t))=-\mathbf{A}(\mathbf{u}(t)) \mathbf{u}(t)+\mathbf{B}(\mathbf{u}(t))[\mathbf{u}(t)], \quad t \in(0, T), \\
\mathbf{u}(t)=\left(\begin{array}{c}
u_{0}(t) \\
u_{1}(t) \\
\vdots \\
u_{m}(t)
\end{array}\right), \quad \mathbf{B}(\mathbf{u}(t))[\mathbf{u}(t)]=\left(\begin{array}{c}
0 \\
\vdots \\
0 \\
\mathscr{B}(\mathbf{u}(t))[\mathbf{u}(t)]
\end{array}\right), \\
\mathbf{A}(\mathbf{u}(t))=\left(\begin{array}{cccccc}
0 & -I & 0 & \ldots & \ldots & 0 \\
\vdots & 0 & -I & 0 & \ldots & \vdots \\
\vdots & & \ddots & \ddots & 0 & \vdots \\
\vdots & & & \ddots & -I & 0 \\
0 & \ldots & \ldots & \ldots & \sum_{i=1}^{r} \mathrm{c}_{i}(\mathbf{u}(t)) \mathscr{A}^{\ell_{i}} & \mathrm{~b}(\mathbf{u}(t)) \mathscr{A}^{k_{m}}
\end{array}\right),
\end{gathered}
$$

where the components of $\mathbf{u}$ represent the solution $u$ and its time derivatives up to order $m$.

Hilbert space setting. In view of the subsequent sections, it is convenient to denote by $(\mathscr{H},(\cdot \mid \cdot),|\cdot|)$ the underlying Lebesgue space $L^{2}(\Omega)$. As common, the inner product of elements in $\mathscr{H}^{m+1}$ is defined componentwise

$$
(\mathbf{h} \mid \mathbf{k})=\sum_{i=0}^{m}\left(h_{i} \mid k_{i}\right), \quad \mathbf{h}=\left(h_{0}, h_{1}, \ldots, h_{m}\right), \mathbf{k}=\left(k_{0}, k_{1}, \ldots, k_{m}\right) \in \mathscr{H}^{m+1} .
$$

We make use of the fact that the negative Laplacian subject to homogeneous Dirichlet boundary conditions is selfadjoint and satisfies a Poincaré-Friedrichs type inequality

$$
\begin{gathered}
\mathscr{A}=-\Delta: \mathscr{D}(\mathscr{A})=H^{2}(\Omega) \cap H_{0}^{1}(\Omega) \subset \mathscr{H} \longrightarrow \mathscr{H}, \\
|v| \leq C_{P F}\left(\left|\mathscr{A}^{1 / 2} v\right|+|\mathscr{A} v|\right), \quad v \in \mathscr{D}(\mathscr{A}) .
\end{gathered}
$$

The unbounded operator

$$
\mathscr{B}: \mathscr{D}_{1}(\mathscr{B}) \subset \mathscr{H}^{m+1} \longrightarrow L\left(\mathscr{D}_{2}(\mathscr{B}), \mathscr{H}\right), \quad \mathscr{D}_{2}(\mathscr{B}) \subset \mathscr{H}^{m+1},
$$

in particular comprises the arising nonlinear terms.

\section{Stiffly Accurate Runge-Kutta Methods}

For the time discretisation of the evolution equation (2.4), we study stiffly accurate RungeKutta methods, see [1,2] and references given therein. We choose time grid points

$$
0=t_{0}<t_{1}<\cdots<t_{N}=T, \quad \tau_{n}=t_{n+1}-t_{n}, \quad n \in\{0,1, \ldots, N-1\},
$$


where the integer number $N>0$ is fixed. Approximations to the values of the exact solution are henceforth denoted by

$$
\mathbf{u}^{(n)}=\left(\begin{array}{c}
u_{0}^{(n)} \\
u_{1}^{(n)} \\
\vdots \\
u_{m}^{(n)}
\end{array}\right) \approx \mathbf{u}\left(t_{n}\right)=\left(\begin{array}{c}
u_{0}\left(t_{n}\right) \\
u_{1}\left(t_{n}\right) \\
\vdots \\
u_{m}\left(t_{n}\right)
\end{array}\right)=\left(\begin{array}{c}
u_{0}\left(t_{n}\right) \\
u_{0}^{\prime}\left(t_{n}\right) \\
\vdots \\
u_{0}^{(m)}\left(t_{n}\right)
\end{array}\right), \quad n \in\{0,1, \ldots, N\}
$$

Implicit Euler method. A well-known instance of a stiffly accurate Runge-Kutta method is the implicit Euler method. For a prescribed initial approximation $\mathbf{u}^{(0)}$, the time-discrete solution to $(2.4)$ is defined by the recurrence

$$
\frac{1}{\tau_{n}}\left(\mathbf{u}^{(n+1)}-\mathbf{u}^{(n)}\right)=\mathbf{F}\left(\mathbf{u}^{(n+1)}\right), \quad n \in\{0,1, \ldots, N-1\} .
$$

In the derivation of energy estimates and global error bounds, we make use of the elementary relation

$$
\left(x_{1}-x_{0}\right) x_{1}=\frac{1}{2}\left(x_{1}^{2}-x_{0}^{2}\right)+\frac{1}{2}\left(x_{1}-x_{0}\right)^{2}, \quad x_{0}, x_{1} \in \mathbb{R},
$$

which carries over to elements of the underlying Hilbert space. In particular, it implies

$$
\begin{aligned}
\left(\mathbf{u}^{(n+1)}-\mathbf{u}^{(n)} \mid \mathbf{u}^{(n+1)}\right)= & \frac{1}{2}\left(\left|\mathbf{u}^{(n+1)}\right|^{2}-\left|\mathbf{u}^{(n)}\right|^{2}\right)+\frac{1}{2}\left|\mathbf{u}^{(n+1)}-\mathbf{u}^{(n)}\right|^{2}, \\
& n \in\{0,1, \ldots, N-1\} .
\end{aligned}
$$

General format. More generally, we consider a consistent stiffly accurate Runge-Kutta method of nonstiff order $p$ with associated Butcher tableau

$$
\begin{aligned}
\frac{\mathfrak{c} \mid}{\mathfrak{A}} \\
\mathfrak{A}=\left(\mathfrak{a}_{i j}\right)_{i, j=1}^{s} \in \mathbb{R}^{s \times s}, \quad \mathfrak{b}=\mathfrak{e}^{T} \mathfrak{A} \in \mathbb{R}^{s}, \quad \mathfrak{e}=(0, \ldots, 0,1)^{T} \in \mathbb{R}^{s}, \quad \mathfrak{c} \in[0,1]^{s} .
\end{aligned}
$$

The stages are determined by a nonlinear system and yield approximations to the exact solution values at the nodes

$$
\begin{gathered}
\frac{1}{\tau_{n}}\left(\mathbf{U}_{i}^{(n)}-\mathbf{u}^{(n)}\right)=\sum_{j=1}^{s} \mathfrak{a}_{i j} \mathbf{F}\left(\mathbf{U}_{j}^{(n)}\right), \\
\mathbf{U}_{i}^{(n)} \approx \mathbf{u}\left(t_{n}+\mathfrak{c}_{i} \tau_{n}\right), \quad i \in\{1,2, \ldots, s\}, \quad n \in\{0,1, \ldots, N-1\} .
\end{gathered}
$$

We recall that the stage order $q$ is characterised as the largest integer number such that

$$
\sum_{j=1}^{s} \mathfrak{a}_{i j} \mathfrak{c}_{j}^{k}=\frac{1}{k+1} \mathfrak{c}_{i}^{k+1}, \quad i \in\{1, \ldots, s\}, \quad k \in\{0,1, \ldots, q-1\}
$$

Compared to general implicit Runge-Kutta methods, a pecularity of stiffly accurate RungeKutta methods is that the weights coincide with the last row of the coefficient matrix and hence the time-discrete solution values are given by the last stages

$$
\mathbf{u}^{(n+1)}=\mathbf{U}_{s}^{(n)} \approx \mathbf{u}\left(t_{n+1}\right), \quad n \in\{0,1, \ldots, N-1\} .
$$


Under the assumption that the matrix

$$
\begin{gathered}
\mathfrak{B} \mathfrak{A}+\mathfrak{A}^{T} \mathfrak{B}-\mathfrak{b} \mathfrak{b}^{T}-\mathfrak{A}^{T} \mathfrak{C}^{\mathfrak{1}} \mathbf{1}^{T} \mathfrak{C}^{T} \mathfrak{A}, \\
\mathfrak{B}=\operatorname{diag}(\mathfrak{b}), \quad \mathfrak{C}=\mathfrak{B} \mathfrak{A}^{-1}, \quad \mathbf{1}=(1, \ldots, 1)^{T} \in \mathbb{R}^{s},
\end{gathered}
$$

is well-defined and positive semidefinite, the inequality

$$
\left(x_{1}, \ldots, x_{s}\right) \mathfrak{C}\left(\begin{array}{c}
x_{1}-x_{0} \\
\vdots \\
x_{s}-x_{0}
\end{array}\right) \geq \frac{1}{2}\left(x_{s}^{2}-x_{0}^{2}\right), \quad x_{0}, x_{1}, \ldots, x_{s} \in \mathbb{R},
$$

is valid, see [1, Lemma 3.4]. This in particular ensures

$$
\sum_{i, j=1}^{s} \mathfrak{C}_{i j}\left(\mathbf{U}_{j}^{(n)}-\mathbf{u}^{(n)} \mid \mathbf{U}_{i}^{(n)}\right) \geq \frac{1}{2}\left(\left|\mathbf{u}^{(n+1)}\right|^{2}-\left|\mathbf{u}^{(n)}\right|^{2}\right), \quad n \in\{0,1, \ldots, N-1\}
$$

Third-order two-stage scheme. As an instance of a higher-order stiffly accurate RungeKutta method fulfilling (3.4), we refer to the Radau IIA method

$$
\begin{gathered}
\mathfrak{a}_{11}=\frac{5}{12}, \quad \mathfrak{a}_{12}=-\frac{1}{12}, \quad \mathfrak{a}_{21}=\frac{3}{4}, \quad \mathfrak{a}_{22}=\frac{1}{4}, \\
\mathfrak{b}_{1}=\mathfrak{a}_{21}=\frac{3}{4}, \quad \mathfrak{b}_{2}=\mathfrak{a}_{22}=\frac{1}{4}, \quad \mathfrak{c}_{1}=\frac{1}{3}, \quad \mathfrak{c}_{2}=1,
\end{gathered}
$$

which has nonstiff order $p=2 s-1=3$ and stage order $q=s=2$. Indeed, the conditions

$$
\begin{gathered}
\sum_{i=1}^{2} \mathfrak{b}_{i}=1, \quad \sum_{i=1}^{2} \mathfrak{b}_{i} \mathfrak{c}_{i}=\frac{1}{2}, \quad \sum_{i=1}^{2} \mathfrak{b}_{i} \mathfrak{c}_{i}^{2}=\frac{1}{3}, \quad \sum_{i=1}^{2} \sum_{j=1}^{2} \mathfrak{b}_{i} \mathfrak{a}_{i j} \mathfrak{c}_{j}=\frac{1}{6}, \\
\sum_{j=1}^{2} \mathfrak{a}_{i j}=\mathfrak{c}_{i}, \quad \sum_{j=1}^{2} \mathfrak{a}_{i j} \mathfrak{c}_{j}=\frac{1}{2} \mathfrak{c}_{i}^{2}, \quad i \in\{1,2\},
\end{gathered}
$$

hold true.

Implicit versus explicit methods. It is remarkable that the special structure of stiffly accurate Runge-Kutta methods permits to prove the inequality (3.4b), which is fundamental for our approach based on the derivation of a priori energy estimates. In view of the introductory remarks and references given in [1], it seems to be possible to extend our analysis to other schemes such as the two-step backward differentiation formula (BDF). However, to our knowledge, the concurrent treatment of a class of methods as presented in this work remains an open question.

We point out that the application of explicit time integration methods to evolution equations involving unbounded operators is not well-defined. For instance, in the context of the the explicit Euler method

$$
\frac{1}{\tau_{n}}\left(\mathbf{u}^{(n+1)}-\mathbf{u}^{(n)}\right)=\mathbf{F}\left(\mathbf{u}^{(n)}\right), \quad n \in\{0,1, \ldots, N-1\},
$$

the analogue of the elementary relation (3.2) reads as

$$
\left(x_{1}-x_{0}\right) x_{1}=\frac{1}{2}\left(x_{1}^{2}-x_{0}^{2}\right)-\frac{1}{2}\left(x_{1}-x_{0}\right)^{2}, \quad x_{0}, x_{1} \in \mathbb{R} .
$$

Due to the inverse sign, the attempt to deduce suitable energy estimates fails. This problem is present in the linear as well as in the nonlinear case. Similar conclusions hold for explicit schemes of Runge-Kutta or linear multistep type. 
Even though a theoretical study of explicit time integration methods within a fully discrete setting is possible, the usefulness of explicit schemes for nonlinear damped wave equations is limited due to stability conditions, which imply severe restrictions on the time stepsizes in dependence of the spatial mesh widths. In particular in the case of three space dimensions, where these limitations significantly confine the practicability of explicit time integration methods, it is expected that stiffly accurate Runge-Kutta methods are beneficial.

Preliminary numerical experiments for explicit schemes applied to linear as well as nonlinear damped wave equations are reported in a talk available at

http://techmath.uibk.ac.at/mecht/MyHomepage/Research/InnsbruckSeptember2020Thalhammer.pdf

(see page 29). For a Gaussian-like initial condition, the classical order of convergence is observed, provided that the chosen time stepsizes are sufficiently small such that stability is ensured. Detailed investigations of full discretisations and numerical comparisons of implicit versus explicit time integration methods will be part of future work.

When the damping parameter tends to zero, the characteristics of the models change considerably. As shown in [31], blow-up in finite time may arise. In this case, a different approach is needed, see [32], and its adaptation to explicit or implicit time discretisation methods, respectively, remains open.

\section{GLOBAL ERror Bounds}

In this section, we deduce global error bounds for stiffly accurate Runge-Kutta methods applied to nonlinear damped wave equations. For this purpose, we presume that the timecontinuous and time-discrete solutions fulfill suitable regularity, consistency, and smallness requirements. These assumptions may be restrictive in view of practical applications. However, in situations, where the initial condition is localised and smooth (e.g., given by a Gaussian-like profile), one may expect high-order convergence in time. Indeed, spatial regularity of higherorder time derivatives of solutions to nonlinear damped wave equations can be established similarly to the standard procedure in [33, Section 7.1.3] under sufficient spatial regularity and compatibility conditions on the initial data.

Time-discrete and time-continuous solutions. Throughout, we assume that the considered stiffly accurate Runge-Kutta method (3.3) satisfies (3.4a) such that in particular the fundamental inequality (3.4b) holds. For a nonlinear damped wave equation cast into the form (2.4), the stages and hence the values of time-discrete solution are determined by the nonlinear system

$$
\frac{1}{\tau_{n}}\left(\mathbf{U}_{i}^{(n)}-\mathbf{u}^{(n)}\right)=\sum_{j=1}^{s} \mathfrak{a}_{i j} \mathbf{F}\left(\mathbf{U}_{j}^{(n)}\right), \quad i \in\{1,2, \ldots, s\}, \quad n \in\{0,1, \ldots, N-1\} .
$$

The corresponding relations for the time-continuous solution read as

$$
t=t_{n}+\mathfrak{c}_{i} \tau_{n}, \quad \mathbf{u}^{\prime}(t)=\mathbf{F}(\mathbf{u}(t)), \quad i \in\{1,2, \ldots, s\}, \quad n \in\{0,1, \ldots, N-1\} .
$$

Errors and defects. The differences between the values of the time-discrete and timecontinuous solutions are denoted by

$$
\begin{gathered}
\mathbf{E}_{i}^{(n)}=\mathbf{U}_{i}^{(n)}-\mathbf{u}\left(t_{n}+\mathfrak{c}_{i} \tau_{n}\right), \quad i \in\{1,2, \ldots, s\}, \quad n \in\{0,1, \ldots, N-1\}, \\
\mathbf{e}^{(n)}=\mathbf{u}^{(n)}-\mathbf{u}\left(t_{n}\right), \quad n \in\{0,1, \ldots, N\} .
\end{gathered}
$$


Inserting the exact solution values into the numerical scheme defines the defects

$$
\begin{aligned}
\frac{1}{\tau_{n}}\left(\mathbf{u}\left(t_{n}+\mathfrak{c}_{i} \tau_{n}\right)-\mathbf{u}\left(t_{n}\right)\right) & =\sum_{j=1}^{s} \mathfrak{a}_{i j} \mathbf{F}\left(\mathbf{u}\left(t_{n}+\mathfrak{c}_{j} \tau_{n}\right)\right)-\mathbf{r}_{i}^{(n)}, \\
i \in\{1,2, \ldots, s\}, & n \in\{0,1, \ldots, N-1\} .
\end{aligned}
$$

In accordance with (3.1) and (4.4), we set

$$
\begin{gathered}
\mathbf{U}_{i}=\left(\begin{array}{c}
U_{i 0}^{(n)} \\
U_{i 1}^{(n)} \\
\vdots \\
U_{i m}^{(n)}
\end{array}\right) \\
i \in\{1,2, \ldots, s\}, \quad \mathbf{E}_{i}^{(n)}=\left(\begin{array}{c}
E_{i 0}^{(n)} \\
E_{i 1}^{(n)} \\
\vdots \\
E_{i m}^{(n)}
\end{array}\right), \quad \mathbf{e}^{(n)}=\left(\begin{array}{c}
e_{0}^{(n)} \\
e_{1}^{(n)} \\
\vdots \\
e_{m}^{(n)}
\end{array}\right), \quad \mathbf{r}_{i}^{(n)}=\left(\begin{array}{c}
r_{i 0}^{(n)} \\
r_{i 1}^{(n)} \\
\vdots \\
r_{i m}^{(n)}
\end{array}\right),
\end{gathered}
$$

Taylor series expansions. Under appropriate regularity assumptions, Taylor series expansions with remainders in integral form yield the representation

$$
\begin{gathered}
\mathbf{u}^{(L)}\left(t_{n}+\mathfrak{c}_{i} \tau_{n}\right)=\sum_{k=0}^{K} \frac{1}{k !} \mathfrak{c}_{i}^{k} \tau_{n}^{k} \mathbf{u}^{(k+L)}\left(t_{n}\right)+\mathfrak{c}_{i}^{K+1} \tau_{n}^{K+1} \mathbf{R}_{K+1}\left(\mathbf{u}^{(K+L+1)}, t_{n}, \mathfrak{c}_{i} \tau_{n}\right), \\
\mathbf{R}_{K+1}\left(\mathbf{u}^{(K+L+1)}, t_{n}, \mathfrak{c}_{i} \tau_{n}\right)=\frac{1}{K !} \int_{0}^{1}(1-\sigma)^{K} \mathbf{u}^{(K+L+1)}\left(t_{n}+\sigma \mathfrak{c}_{i} \tau_{n}\right) \mathrm{d} \sigma \\
i \in\{1,2, \ldots, s\}, \quad n \in\{0,1, \ldots, N-1\}, \quad K, L \in \mathbb{N}_{0} .
\end{gathered}
$$

Replacing in (4.3) the defining operator by the time-derivative of the solution and recalling the stage order conditions (3.3c), this implies

$$
\begin{aligned}
\mathbf{r}_{i}^{(n)}= & \sum_{j=1}^{s} \mathfrak{a}_{i j} \mathbf{F}\left(\mathbf{u}\left(t_{n}+\mathfrak{c}_{j} \tau_{n}\right)\right)-\frac{1}{\tau_{n}}\left(\mathbf{u}\left(t_{n}+\mathfrak{c}_{i} \tau_{n}\right)-\mathbf{u}\left(t_{n}\right)\right) \\
= & \sum_{j=1}^{s} \mathfrak{a}_{i j} \mathbf{u}^{\prime}\left(t_{n}+\mathfrak{c}_{j} \tau_{n}\right)-\frac{1}{\tau_{n}}\left(\mathbf{u}\left(t_{n}+\mathfrak{c}_{i} \tau_{n}\right)-\mathbf{u}\left(t_{n}\right)\right) \\
= & \sum_{k=0}^{q-1} \frac{1}{k !} \tau_{n}^{k}\left(\sum_{j=1}^{s} \mathfrak{a}_{i j} \mathfrak{c}_{j}^{k}-\frac{1}{k+1} \mathfrak{c}_{i}^{k+1}\right) \mathbf{u}^{(k+1)}\left(t_{n}\right) \\
& \quad+\tau_{n}^{q}\left(\sum_{j=1}^{s} \mathfrak{a}_{i j} \mathfrak{c}_{j}^{q} \mathbf{R}_{q}\left(\mathbf{u}^{(q+1)}, t_{n}, \mathfrak{c}_{j} \tau_{n}\right)-\mathfrak{c}_{i}^{q+1} \mathbf{R}_{q+1}\left(\mathbf{u}^{(q+1)}, t_{n}, \mathfrak{c}_{i} \tau_{n}\right)\right) \\
= & \tau_{n}^{q}\left(\sum_{j=1}^{s} \mathfrak{a}_{i j} \mathfrak{c}_{j}^{q} \mathbf{R}_{q}\left(\mathbf{u}^{(q+1)}, t_{n}, \mathfrak{c}_{j} \tau_{n}\right)\right. \\
& \left.\quad-\mathfrak{c}_{i}^{q+1} \mathbf{R}_{q+1}\left(\mathbf{u}^{(q+1)}, t_{n}, \mathfrak{c}_{i} \tau_{n}\right)\right), \quad i \in\{1,2, \ldots, s\}, \quad n \in\{0,1, \ldots, N-1\}
\end{aligned}
$$

Hence, boundedness of the defects is linked to boundedness of certain time derivatives of the solution in the underlying Hilbert space

$$
\begin{gathered}
\left|\mathbf{r}_{i}^{(n)}\right| \leq C \tau_{n}^{q}|\mathbf{u}|_{W_{\infty}^{q+1}\left(0, T ; \mathscr{C}^{m+1}\right)}, \quad\left|r_{i j}^{(n)}\right| \leq C \tau_{n}^{q}\left|u_{0}\right|_{W_{\infty}^{q+j+1}(0, T ; \mathscr{H})}, \quad j \in\{0,1, \ldots, m\}, \\
i \in\{1,2, \ldots, s\}, \quad n \in\{0,1, \ldots, N-1\} .
\end{gathered}
$$


The generic constant $C>0$ depends on the coefficients of the considered stiffly accurate RungeKutta method.

Error relation. Our starting point for the derivation of a global error bound is the relation

$$
\begin{gathered}
\frac{1}{\tau_{n}}\left(\mathbf{E}_{i}^{(n)}-\mathbf{e}^{(n)}\right)=\sum_{j=1}^{s} \mathfrak{a}_{i j}\left(\mathbf{F}\left(\mathbf{U}_{j}^{(n)}\right)-\mathbf{F}\left(\mathbf{u}\left(t_{n}+\mathfrak{c}_{j} \tau_{n}\right)\right)\right)+\mathbf{r}_{i}^{(n)}, \\
i \in\{1,2, \ldots, s\}, \quad n \in\{0,1, \ldots, N-1\},
\end{gathered}
$$

which results from (4.1) and (4.3). In view of (3.4), it is essential to employ the reformulation

$$
\begin{gathered}
\sum_{j=1}^{s} \mathfrak{C}_{i j}\left(\mathbf{E}_{j}^{(n)}-\mathbf{e}^{(n)}\right)=\tau_{n} \mathfrak{b}_{i}\left(\mathbf{F}\left(\mathbf{U}_{i}^{(n)}\right)-\mathbf{F}\left(\mathbf{u}\left(t_{n}+\mathfrak{c}_{i} \tau_{n}\right)\right)+\tau_{n} \sum_{j=1}^{s} \mathfrak{C}_{i j} \mathbf{r}_{j}^{(n)},\right. \\
i \in\{1,2, \ldots, s\}, \quad n \in\{0,1, \ldots, N-1\} .
\end{gathered}
$$

In addition, we make use of the integral representation

$$
\begin{gathered}
\mathbf{F}\left(\mathbf{U}_{i}^{(n)}\right)-\mathbf{F}\left(\mathbf{u}\left(t_{n}+\mathfrak{c}_{i} \tau_{n}\right)\right)=\int_{0}^{1} \mathbf{F}^{\prime}\left(\sigma \mathbf{U}_{i}^{(n)}+(1-\sigma) \mathbf{u}\left(t_{n}+\mathfrak{c}_{i} \tau_{n}\right)\right) \mathrm{d} \sigma \mathbf{E}_{i}^{(n)}, \\
i \in\{1,2, \ldots, s\}, \quad n \in\{0,1, \ldots, N-1\} .
\end{gathered}
$$

Altogether, this yields the error relation

$$
\begin{gathered}
\sum_{j=1}^{s} \mathfrak{C}_{i j}\left(\mathbf{E}_{j}^{(n)}-\mathbf{e}^{(n)}\right)=\tau_{n} \mathfrak{b}_{i}\left(\int_{0}^{1} \mathbf{F}^{\prime}\left(\sigma \mathbf{U}_{i}^{(n)}+(1-\sigma) \mathbf{u}\left(t_{n}+\mathfrak{c}_{i} \tau_{n}\right)\right) \mathrm{d} \sigma \mathbf{E}_{i}^{(n)}\right)+\tau_{n} \sum_{j=1}^{s} \mathfrak{C}_{i j} \mathbf{r}_{j}^{(n)}, \\
i \in\{1,2, \ldots, s\}, \quad n \in\{0,1, \ldots, N-1\} .
\end{gathered}
$$

Specifically, for the implicit Euler method with $p=q=s=\mathfrak{C}_{11}=\mathfrak{b}_{1}=\mathfrak{c}_{1}=1$, this relation reduces to

$$
\begin{aligned}
\left(\mathbf{e}^{(n+1)}-\mathbf{e}^{(n)}\right)= & \tau_{n}\left(\int_{0}^{1} \mathbf{F}^{\prime}\left(\sigma \mathbf{u}^{(n+1)}+(1-\sigma) \mathbf{u}\left(t_{n+1}\right) \mathrm{d} \sigma \mathbf{e}^{(n+1)}\right)+\tau_{n} \mathbf{r}_{1}^{(n)},\right. \\
& i \in\{1,2, \ldots, s\}, \quad n \in\{0,1, \ldots, N-1\} .
\end{aligned}
$$

4.1. Westervelt equation. We first focus on the derivation of a global error bound for stiffly accurate Runge-Kutta methods applied to the simplest model, the Westervelt equation. For (2.1), the arising nonlinear operator and its Fréchet derivative are given by

$$
\begin{aligned}
& \mathbf{v}=\left(v_{0}, v_{1}\right), \quad \mathbf{F}(\mathbf{v})=\left(\begin{array}{c}
v_{1} \\
F_{2}(\mathbf{v})
\end{array}\right), \quad \mathbf{F}^{\prime}(\mathbf{v})=\left(\begin{array}{cc}
0 & I \\
F_{21}^{\prime}(\mathbf{v}) & F_{22}^{\prime}(\mathbf{v})
\end{array}\right) \\
& F_{2}(\mathbf{v})=\left(1-\beta_{3} v_{0}\right)^{-1}\left(-\beta_{1} \mathscr{A} v_{0}-\beta_{2} \mathscr{A} v_{1}+\beta_{3} v_{1}^{2}\right), \\
& F_{21}^{\prime}(\mathbf{v})=f_{11}(\mathbf{v})-f_{12}(\mathbf{v}) \mathscr{A}, \quad F_{22}^{\prime}(\mathbf{v})=f_{21}(\mathbf{v})-f_{22}(\mathbf{v}) \mathscr{A}, \\
& f_{11}(\mathbf{v})=\beta_{3}\left(1-\beta_{3} v_{0}\right)^{-2}\left(-\beta_{1} \mathscr{A} v_{0}-\beta_{2} \mathscr{A} v_{1}+\beta_{3} v_{1}^{2}\right), \quad f_{12}(\mathbf{v})=\beta_{1}\left(1-\beta_{3} v_{0}\right)^{-1} \text {, } \\
& f_{21}(\mathbf{v})=2 \beta_{3}\left(1-\beta_{3} v_{0}\right)^{-1} v_{1}, \quad f_{22}(\mathbf{v})=\beta_{2}\left(1-\beta_{3} v_{0}\right)^{-1},
\end{aligned}
$$


with $\mathscr{A}$ representing the negative Laplacian subject to homogeneous boundary conditions and $\beta_{1}, \beta_{2}, \beta_{3}>0$ denoting certain constants. An essential observation is that regularity and nondegeneracy ensure bounds of the form

$$
\begin{aligned}
& \max _{\ell \in\{11,12,21,22\}} \max _{\substack{i \in\{1,2, \ldots, S\} \\
n \in\{1,2, \ldots, N\}}}\left|\int_{0}^{1} f_{\ell}\left(\sigma \mathbf{U}_{i}^{(n)}+(1-\sigma) \mathbf{u}\left(t_{n}+\mathfrak{c}_{i} \tau_{n}\right)\right) \mathrm{d} \sigma\right|_{L^{\infty}(\Omega)} \\
& +\max _{\ell \in\{12,22\}} \max _{\substack{i \in\{1,2, \ldots, s\} \\
n \in\{1,2, \ldots, N\}}}\left|\int_{0}^{1} f_{\ell}\left(\sigma \mathbf{U}_{i}^{(n)}+(1-\sigma) \mathbf{u}\left(t_{n}+\mathfrak{c}_{i} \tau_{n}\right)\right) \mathrm{d} \sigma\right|_{W_{\infty}^{1}(\Omega)} \leq C_{u}, \\
& C_{u}=C_{u}\left(\max _{\substack{i \in\{1,2, \ldots, s\} \\
n \in\{1,2, \ldots, N\}}}\left|\mathbf{U}_{i}^{(n)}\right|_{\left(W_{\infty}^{2}(\Omega)\right)^{2}},|\mathbf{u}|_{L^{\infty}\left(0, T ;\left(W_{\infty}^{2}(\Omega)\right)^{2}\right)}\right),
\end{aligned}
$$

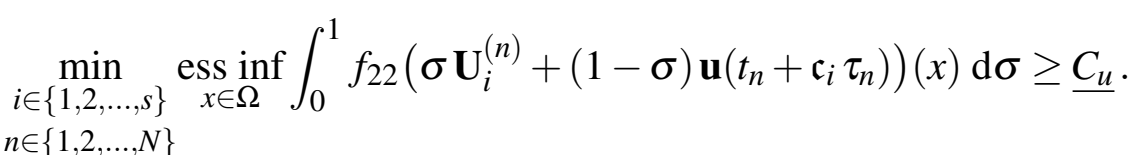

Weak formulation. We test the error relation stated above with

$$
\begin{gathered}
\mathcal{A} \mathbf{E}_{i}^{(n)}, \quad i \in\{1,2, \ldots, s\}, \quad n \in\{0,1, \ldots, N-1\}, \\
\mathcal{A}=\operatorname{diag}\left(\mathscr{A}^{k+1}, \mathscr{A}^{k}\right), \quad k \in\{0,1\},
\end{gathered}
$$

perform integration-by-parts, and add all components to obtain

$$
\begin{aligned}
\sum_{i, j=1}^{s} \mathfrak{C}_{i j} & \left(\mathcal{A}^{1 / 2}\left(\mathbf{E}_{j}^{(n)}-\mathbf{e}^{(n)}\right) \mid \mathcal{A}^{1 / 2} \mathbf{E}_{i}^{(n)}\right) \\
=\tau_{n} & \sum_{i=1}^{s} \mathfrak{b}_{i}\left(\int_{0}^{1} \mathbf{F}^{\prime}\left(\sigma \mathbf{U}_{i}^{(n)}+(1-\sigma) \mathbf{u}\left(t_{n}+\mathfrak{c}_{i} \tau_{n}\right)\right) \mathrm{d} \sigma \mathbf{E}_{i}^{(n)} \mid \mathcal{A} \mathbf{E}_{i}^{(n)}\right) \\
& +\tau_{n} \sum_{i, j=1}^{s} \mathfrak{C}_{i j}\left(\mathcal{A}^{1 / 2} \mathbf{r}_{j}^{(n)} \mid \mathcal{A}^{1 / 2} \mathbf{E}_{i}^{(n)}\right), \quad n \in\{0,1, \ldots, N-1\} .
\end{aligned}
$$

The elementary estimate given in (3.4), Young's inequality with additional weight

$$
x_{1} x_{2} \leq C_{Y}(\varepsilon) x_{1}^{2}+C_{Y}\left(\frac{1}{\varepsilon}\right) x_{2}^{2}, \quad x_{1}, x_{2} \in \mathbb{R}, \quad C_{Y}(\varepsilon)=\frac{1}{2} \varepsilon, \quad C_{Y}\left(\frac{1}{\varepsilon}\right)=\frac{1}{2} \frac{1}{\varepsilon}, \quad \varepsilon>0,
$$

and the expansion of the defects (4.4) imply

$$
\begin{aligned}
& \left|\mathcal{A}^{1 / 2} \mathbf{e}^{(n+1)}\right|^{2}-\left|\mathcal{A}^{1 / 2} \mathbf{e}^{(n)}\right|^{2} \\
& \leq \varepsilon_{0} \tau_{n} \sum_{i=1}^{s}\left|\mathcal{A}^{1 / 2} \mathbf{E}_{i}^{(n)}\right|^{2} \\
& \quad+2 \tau_{n} \sum_{i=1}^{s} \mathfrak{b}_{i}\left(\int_{0}^{1} \mathbf{F}^{\prime}\left(\sigma \mathbf{U}_{i}^{(n)}+(1-\sigma) \mathbf{u}\left(t_{n}+\mathfrak{c}_{i} \tau_{n}\right)\right) \mathrm{d} \sigma \mathbf{E}_{i}^{(n)} \mid \mathcal{A} \mathbf{E}_{i}^{(n)}\right) \\
& \quad+C \frac{1}{\varepsilon_{0}}\left|\mathcal{A}^{1 / 2} \mathbf{u}\right|_{W_{\infty}^{q+1}\left(0, T ;\left(L^{2}(\Omega)\right)^{2}\right)}^{2} \tau_{n}^{2 q+1}, \quad n \in\{0,1, \ldots, N-1\},
\end{aligned}
$$


with $\varepsilon_{0}>0$ and generic constant $C>0$ depending on the method coefficients. We note that the special form of the defining operator implies

$$
\begin{gathered}
\mathscr{A}^{k / 2} E_{i 0}^{(n)}=\mathscr{A}^{k / 2} e_{0}^{(n)}+\tau_{n} \sum_{j=1}^{s} a_{i j} \mathscr{A}^{k / 2} E_{j 1}^{(n)}+\tau_{n} \mathscr{A}^{k / 2} r_{i 0}^{(n)}, \\
i \in\{1,2, \ldots, s\}, \quad n \in\{0,1, \ldots, N-1\}, \quad k \in \mathbb{N}_{0} .
\end{gathered}
$$

see (4.5). By means of Young's inequality and the estimate for the defects (4.4), we thus obtain the bound

$$
\begin{aligned}
& \left|\mathscr{A}^{k / 2} E_{i 0}^{(n)}\right|^{2} \\
& \quad \leq 3\left|\mathscr{A}^{k / 2} e_{0}^{(n)}\right|^{2}+C \tau_{n} \sum_{j=1}^{s}\left|\mathscr{A}^{k / 2} E_{j 1}^{(n)}\right|^{2}+C \tau_{n}^{2 q+1}\left|\mathscr{A}^{k / 2} u_{0}\right|_{W_{\infty}^{q+1}(0, T ; \mathscr{H})}^{2}, \\
& \quad i \in\{1,2, \ldots, s\}, \quad n \in\{0,1, \ldots, N-1\}, \quad k \in \mathbb{N}_{0},
\end{aligned}
$$

with a generic constant $C>0$ that depends on the coefficients of the considered stiffly accurate Runge-Kutta method. We next deduce an auxiliary estimate for the term

$$
\begin{gathered}
\left(\int_{0}^{1} \mathbf{F}^{\prime}\left(\sigma \mathbf{U}_{i}^{(n)}+(1-\sigma) \mathbf{u}\left(t_{n}+\mathfrak{c}_{i} \tau_{n}\right)\right) \mathrm{d} \sigma \mathbf{E}_{i}^{(n)} \mid \mathcal{A} \mathbf{E}_{i}^{(n)}\right), \\
i \in\{1,2, \ldots, s\}, \quad n \in\{0,1, \ldots, N-1\}
\end{gathered}
$$

Auxiliary estimate. Let $i \in\{1,2, \ldots, s\}$ and $n \in\{0,1, \ldots, N-1\}$. Our starting point is the equality

$$
\begin{aligned}
\left(\int_{0}^{1} \mathbf{F}^{\prime}\right. & \left.\left.\left(\sigma \mathbf{U}_{i}^{(n)}+(1-\sigma) \mathbf{u}\left(t_{n}+\mathfrak{c}_{i} \tau_{n}\right)\right) \mathrm{d} \sigma \mathbf{E}_{i}^{(n)}\right) \mid \mathcal{A} \mathbf{E}_{i}^{(n)}\right) \\
=( & \left.E_{i 1}^{(n)} \mid \mathscr{A}_{0} E_{i 0}^{(n)}\right) \\
& +\left(\int_{0}^{1} f_{11}\left(\sigma \mathbf{U}_{i}^{(n)}+(1-\sigma) \mathbf{u}\left(t_{n}+\mathfrak{c}_{i} \tau_{n}\right)\right) \mathrm{d} \sigma E_{i 0}^{(n)} \mid \mathscr{A}_{1} E_{i 1}^{(n)}\right) \\
& -\left(\int_{0}^{1} f_{12}\left(\sigma \mathbf{U}_{i}^{(n)}+(1-\sigma) \mathbf{u}\left(t_{n}+\mathfrak{c}_{i} \tau_{n}\right)\right) \mathrm{d} \sigma \mathscr{A} E_{i 0}^{(n)} \mid \mathscr{A}_{1} E_{i 1}^{(n)}\right) \\
& +\left(\int_{0}^{1} f_{21}\left(\sigma \mathbf{U}_{i}^{(n)}+(1-\sigma) \mathbf{u}\left(t_{n}+\mathfrak{c}_{i} \tau_{n}\right)\right) \mathrm{d} \sigma E_{i 1}^{(n)} \mid \mathscr{A}_{1} E_{i 1}^{(n)}\right) \\
& -\left(\int_{0}^{1} f_{22}\left(\sigma \mathbf{U}_{i}^{(n)}+(1-\sigma) \mathbf{u}\left(t_{n}+\mathfrak{c}_{i} \tau_{n}\right)\right) \mathrm{d} \sigma \mathscr{A} E_{i 1}^{(n)} \mid \mathscr{A}_{1} E_{i 1}^{(n)}\right)
\end{aligned}
$$

and reformulations resulting from integration-by-parts.

(i) We first consider the case

$$
k=1, \quad \mathscr{A}_{0}=\mathscr{A}^{2}, \quad \mathscr{A}_{1}=\mathscr{A},
$$


where the above given relation rewrites as

$$
\begin{aligned}
\left(\int_{0}^{1} \mathbf{F}^{\prime}\right. & \left.\left(\sigma \mathbf{U}_{i}^{(n)}+(1-\sigma) \mathbf{u}\left(t_{n}+\mathfrak{c}_{i} \tau_{n}\right)\right) \mathrm{d} \sigma \mathbf{E}_{i}^{(n)} \mid \mathcal{A} \mathbf{E}_{i}^{(n)}\right) \\
=\left(\mathscr{A} E_{i 1}^{(n)} \mid \mathscr{A} E_{i 0}^{(n)}\right) & \\
& +\left(\int_{0}^{1} f_{11}\left(\sigma \mathbf{U}_{i}^{(n)}+(1-\sigma) \mathbf{u}\left(t_{n}+\mathfrak{c}_{i} \tau_{n}\right)\right) \mathrm{d} \sigma E_{i 0}^{(n)} \mid \mathscr{A} E_{i 1}^{(n)}\right) \\
& -\left(\int_{0}^{1} f_{12}\left(\sigma \mathbf{U}_{i}^{(n)}+(1-\sigma) \mathbf{u}\left(t_{n}+\mathfrak{c}_{i} \tau_{n}\right)\right) \mathrm{d} \sigma \mathscr{A} E_{i 0}^{(n)} \mid \mathscr{A} E_{i 1}^{(n)}\right) \\
& +\left(\int_{0}^{1} f_{21}\left(\sigma \mathbf{U}_{i}^{(n)}+(1-\sigma) \mathbf{u}\left(t_{n}+\mathfrak{c}_{i} \tau_{n}\right)\right) \mathrm{d} \sigma E_{i 1}^{(n)} \mid \mathscr{A} E_{i 1}^{(n)}\right) \\
& -\left(\int_{0}^{1} f_{22}\left(\sigma \mathbf{U}_{i}^{(n)}+(1-\sigma) \mathbf{u}\left(t_{n}+\mathfrak{c}_{i} \tau_{n}\right)\right) \mathrm{d} \sigma \mathscr{A} E_{i 1}^{(n)} \mid \mathscr{A} E_{i 1}^{(n)}\right) .
\end{aligned}
$$

By means of Young's inequality with weight $\varepsilon_{1}>0$ and the Poincaré-Friedrichs type inequality (2.5), we obtain

$$
\begin{aligned}
& \left(\int_{0}^{1} \mathbf{F}^{\prime}\left(\sigma \mathbf{U}_{i}^{(n)}+(1-\sigma) \mathbf{u}\left(t_{n}+\mathfrak{c}_{i} \tau_{n}\right)\right) \mathrm{d} \sigma \mathbf{E}_{i}^{(n)} \mid \mathcal{A} \mathbf{E}_{i}^{(n)}\right) \\
& \quad \leq\left(1+C C_{u}\right)\left|\mathscr{A} E_{i 0}^{(n)}\right|\left|\mathscr{A} E_{i 1}^{(n)}\right|+C_{u}\left|E_{i 1}^{(n)}\right|\left|\mathscr{A} E_{i 1}^{(n)}\right|-\underline{C_{u}}\left|\mathscr{A} E_{i 1}^{(n)}\right|^{2} \\
& \quad \leq \frac{1}{\varepsilon_{1}}\left(1+C C_{u}\right)\left|\mathscr{A} E_{i 0}^{(n)}\right|^{2}+\frac{1}{\varepsilon_{1}} C_{u}\left|E_{i 1}^{(n)}\right|^{2}+\left(\varepsilon_{1}\left(1+C C_{u}\right)-\underline{C_{u}}\right)\left|\mathscr{A} E_{i 1}^{(n)}\right|^{2}
\end{aligned}
$$

with generic constant $C>0$ depending on $C_{P F}>0$. Provided that the weight $\varepsilon_{1}>0$ is chosen sufficiently small such that

$$
\varepsilon_{1}\left(1+C C_{u}\right)-\underline{C_{u}} \leq-\delta<0
$$

we arrive at the estimate

$$
\begin{gathered}
k=1, \quad \mathscr{A}_{0}=\mathscr{A}^{2}, \quad \mathscr{A}_{1}=\mathscr{A} \\
\left(\int_{0}^{1} \mathbf{F}^{\prime}\left(\sigma \mathbf{U}_{i}^{(n)}+(1-\sigma) \mathbf{u}\left(t_{n}+\mathfrak{c}_{i} \tau_{n}\right)\right) \mathrm{d} \sigma \mathbf{E}_{i}^{(n)} \mid \mathcal{A} \mathbf{E}_{i}^{(n)}\right) \\
\leq \frac{1}{\varepsilon_{1}}\left(1+C C_{u}\right)\left|\mathscr{A} E_{i 0}^{(n)}\right|^{2}+\frac{1}{\varepsilon_{1}} C_{u}\left|E_{i 1}^{(n)}\right|^{2}-\delta\left|\mathscr{A} E_{i 1}^{(n)}\right|^{2} .
\end{gathered}
$$

(ii) Similar arguments are employed in the case

$$
k=0, \quad \mathscr{A}_{0}=\mathscr{A}, \quad \mathscr{A}_{1}=I .
$$


Here, applying integration-by-parts, Young's inequality with weight $\varepsilon_{1}>0$, and the PoincaréFriedrichs type inequality (2.5), yields

$$
\begin{aligned}
\left(\int_{0}^{1} \mathbf{F}^{\prime}\right. & \left.\left(\sigma \mathbf{U}_{i}^{(n)}+(1-\sigma) \mathbf{u}\left(t_{n}+\mathfrak{c}_{i} \tau_{n}\right)\right) \mathrm{d} \sigma \mathbf{E}_{i}^{(n)} \mid \mathcal{A} \mathbf{E}_{i}^{(n)}\right) \\
=( & \left.\mathscr{A}^{1 / 2} E_{i 1}^{(n)} \mid \mathscr{A}^{1 / 2} E_{i 0}^{(n)}\right) \\
& +\left(\int_{0}^{1} f_{11}\left(\sigma \mathbf{U}_{i}^{(n)}+(1-\sigma) \mathbf{u}\left(t_{n}+\mathfrak{c}_{i} \tau_{n}\right)\right) \mathrm{d} \sigma E_{i 0}^{(n)} \mid E_{i 1}^{(n)}\right) \\
& -\left(\mathscr{A}^{1 / 2} E_{i 0}^{(n)} \mid \mathscr{A}^{1 / 2}\left(\int_{0}^{1} f_{12}\left(\sigma \mathbf{U}_{i}^{(n)}+(1-\sigma) \mathbf{u}\left(t_{n}+\mathfrak{c}_{i} \tau_{n}\right)\right) \mathrm{d} \sigma E_{i 1}^{(n)}\right)\right) \\
& +\left(\int_{0}^{1} f_{21}\left(\sigma \mathbf{U}_{i}^{(n)}+(1-\sigma) \mathbf{u}\left(t_{n}+\mathfrak{c}_{i} \tau_{n}\right)\right) \mathrm{d} \sigma E_{i 1}^{(n)} \mid E_{i 1}^{(n)}\right) \\
& -\left(\mathscr{A}^{1 / 2} E_{i 1}^{(n)} \mid \mathscr{A}^{1 / 2}\left(\int_{0}^{1} f_{22}\left(\sigma \mathbf{U}_{i}^{(n)}+(1-\sigma) \mathbf{u}\left(t_{n}+\mathfrak{c}_{i} \tau_{n}\right)\right) \mathrm{d} \sigma E_{i 1}^{(n)}\right)\right) \\
\leq\left|\mathscr{A}^{1 / 2} E_{i 0}^{(n)}\right|\left|\mathscr{A}^{1 / 2} E_{i 1}^{(n)}\right|+C_{u}\left(\left|E_{i 0}^{(n)}\right|\left|E_{i 1}^{(n)}\right|+\left|\mathscr{A}^{1 / 2} E_{i 0}^{(n)}\right|\left|\mathscr{A}^{1 / 2} E_{i 1}^{(n)}\right|\right. & \\
& \left.+\left|\mathscr{A}^{1 / 2} E_{i 0}^{(n)}\right|\left|E_{i 1}^{(n)}\right|+\left|E_{i 1}^{(n)}\right|^{2}+\left|E_{i 1}^{(n)}\right|\left|\mathscr{A}^{1 / 2} E_{i 1}^{(n)}\right|\right)-C_{u}\left|\mathscr{A}^{1 / 2} E_{i 1}^{(n)}\right|^{2} \\
\leq & \frac{1}{\varepsilon_{1}}\left(1+C_{u}\right)\left|\mathscr{A}^{1 / 2} E_{i 0}^{(n)}\right|^{2}+\left(1+\frac{1}{\varepsilon_{1}}\right) C_{u}\left|E_{i 1}^{(n)}\right|^{2} \\
& +\left(\varepsilon_{1}\left(1+C_{u}\right)-C_{u}\right)\left|\mathscr{A}^{1 / 2} E_{i 1}^{(n)}\right|^{2}
\end{aligned}
$$

with generic constant $C>0$ depending on $C_{P F}>0$. Hence, by choosing the weight $\varepsilon_{1}>0$ sufficiently small such that

$$
\varepsilon_{1}\left(1+C C_{u}\right)-\underline{C_{u}} \leq-\delta<0,
$$

we get the estimate

$$
\begin{gathered}
k=0, \quad \mathscr{A}_{0}=\mathscr{A}, \quad \mathscr{A}_{1}=I \\
\left(\int_{0}^{1} \mathbf{F}^{\prime}\left(\sigma \mathbf{U}_{i}^{(n)}+(1-\sigma) \mathbf{u}\left(t_{n}+\mathfrak{c}_{i} \tau_{n}\right)\right) \mathrm{d} \sigma \mathbf{E}_{i}^{(n)} \mid \mathcal{A} \mathbf{E}_{i}^{(n)}\right) \\
\leq \frac{1}{\varepsilon_{1}}\left(1+C C_{u}\right)\left|\mathscr{A}^{1 / 2} E_{i 0}^{(n)}\right|^{2}+\left(1+\frac{1}{\varepsilon_{1}}\right) C_{u}\left|E_{i 1}^{(n)}\right|^{2}-\delta\left|\mathscr{A}^{1 / 2} E_{i 1}^{(n)}\right|^{2} .
\end{gathered}
$$

Global error bound (Implicit Euler method). As illustration, we first consider the implicit Euler method, where it suffices to set $\varepsilon_{0}=1$ and the above stated arguments show

$$
\begin{aligned}
& \left|\mathcal{A}^{1 / 2} \mathbf{e}^{(n+1)}\right|^{2}-\left|\mathcal{A}^{1 / 2} \mathbf{e}^{(n)}\right|^{2} \\
& \quad \leq C C_{1}\left(\frac{1}{\varepsilon_{1}}, C_{u}\right) \tau_{n}\left|\mathcal{A}^{1 / 2} \mathbf{e}^{(n+1)}\right|^{2}+C\left|\mathcal{A}^{1 / 2} \mathbf{u}\right|_{W_{\infty}^{2}\left(0, T ;\left(L^{2}(\Omega)\right)^{2}\right)} \tau_{n}^{3}, \quad n \in\{0,1, \ldots, N-1\}
\end{aligned}
$$

with generic constants and $C_{1}$ depending on the indicated quantitites, see also (4.6), (4.8) and (4.9). Summation and a telescopic identity imply

$$
\begin{aligned}
& \left|\mathcal{A}^{1 / 2} \mathbf{e}^{(N)}\right|^{2} \\
& \quad \leq\left|\mathcal{A}^{1 / 2} \mathbf{e}^{(0)}\right|^{2}+C C_{1}\left(\frac{1}{\varepsilon_{1}}, C_{u}\right) \sum_{n=0}^{N-1} \tau_{n}\left|\mathcal{A}^{1 / 2} \mathbf{e}^{(n+1)}\right|^{2}+C\left|\mathcal{A}^{1 / 2} \mathbf{u}\right|_{W_{\infty}^{2}\left(0, T ;\left(L^{2}(\Omega)\right)^{2}\right)} \sum_{n=0}^{N-1} \tau_{n}^{3} .
\end{aligned}
$$


Finally, by a Gronwall inequality, we obtain the global error bound

$$
\begin{aligned}
\left|\mathcal{A}^{1 / 2} \mathbf{e}^{(N)}\right|^{2} & \leq C C_{1}\left(\frac{1}{\varepsilon_{1}}, C_{u}\right)\left(\left|\mathcal{A}^{1 / 2} \mathbf{e}^{(0)}\right|^{2}+\tau_{\max }^{2}\right), \\
\tau_{\max } & =\max \left\{\tau_{n}: n \in\{0,1, \ldots, N-1\}\right\},
\end{aligned}
$$

where the arising constant in addition depends on the final time.

Global error bound (Stiffly accurate Runge-Kutta method). More generally, combining (4.6), (4.7), (4.8), and (4.9), a bound of the form

$$
\begin{aligned}
& \left|\mathcal{A}^{1 / 2} \mathbf{e}^{(n+1)}\right|^{2}-\left|\mathcal{A}^{1 / 2} \mathbf{e}^{(n)}\right|^{2}+\tau_{n} \sum_{i=1}^{s}\left|\mathscr{A}^{(k+1) / 2} E_{i 1}^{(n)}\right|^{2} \\
& \leq C \tau_{n} \sum_{i=1}^{s}\left|\mathcal{A}^{1 / 2} \mathbf{e}^{(n)}\right|^{2} \\
& \quad+C \tau_{n}\left(\varepsilon_{0}+\tau_{n}+C_{u}\right) \sum_{i=1}^{s}\left|\mathscr{A}^{(k+1) / 2} E_{i 1}^{(n)}\right|^{2} \\
& \quad+C\left|\mathcal{A}^{1 / 2} \mathbf{u}\right|_{W_{\infty}^{q+1}\left(0, T ;\left(L^{2}(\Omega)\right)^{2}\right.}^{2} \tau_{n}^{2 q+1}, \quad n \in\{0,1, \ldots, N-1\},
\end{aligned}
$$

holds. Requiring the time stepsizes, the weight $\varepsilon_{0}>0$, the initial data and hence the bound $C_{u}$ to be sufficiently small, we have

$$
\begin{aligned}
& \left|\mathcal{A}^{1 / 2} \mathbf{e}^{(n+1)}\right|^{2}-\left|\mathcal{A}^{1 / 2} \mathbf{e}^{(n)}\right|^{2} \\
& \quad \leq C \tau_{n} \sum_{i=1}^{s}\left|\mathcal{A}^{1 / 2} \mathbf{e}^{(n)}\right|^{2}+C\left|\mathcal{A}^{1 / 2} \mathbf{u}\right|_{W_{\infty}^{q+1}\left(0, T ;\left(L^{2}(\Omega)\right)^{2}\right.}^{2} \tau_{n}^{2 q+1}, \quad n \in\{0,1, \ldots, N-1\} .
\end{aligned}
$$

Thus, summation and a Gronwall inequality lead to the result

$$
\begin{gathered}
\left|\mathcal{A}^{1 / 2} \mathbf{e}^{(N)}\right|_{\left(L^{2}(\Omega)\right)^{2}}^{2} \leq C\left(\left|\mathcal{A}^{1 / 2} \mathbf{e}^{(0)}\right|_{\left(L^{2}(\Omega)\right)^{2}}^{2}+\tau_{\max }^{2 q}\right), \\
\tau_{\max }=\max \left\{\tau_{n}: n \in\{0,1, \ldots, N-1\}\right\} .
\end{gathered}
$$

Altogether, we arrive at the following result.

Theorem 4.1 (Westervelt equation). Let $\mathbf{u}=\left(u_{0}, u_{1}\right)^{T}=\left(u, u^{\prime}\right)^{T}$ denote the solution to (2.1), and set $\mathcal{A}=\operatorname{diag}\left(\mathscr{A}^{k+1}, \mathscr{A}^{k}\right)$ for $k \in\{0,1\}$. Suppose that the considered stiffly accurate Runge-Kutta method of nonstiff order $p$ and stage order $q$ fulfills the fundamental condition (3.4). Provided that the time-continuous and time-discrete solutions satisfy the regularity requirements

$$
\begin{gathered}
\left|u_{0}\right|_{W_{\infty}^{1}\left(0, T ; W_{\infty}^{2}(\Omega)\right)}+\left|u_{0}\right|_{W_{\infty}^{r+1}\left(0, T ; W_{2}^{1}(\Omega)\right)}+\left|u_{0}\right|_{W_{\infty}^{r+2}\left(0, T ; L^{2}(\Omega)\right)} \leq C, \quad r=\min \{p, q\}, \\
\max \left\{\left|\mathbf{U}_{i}^{(n)}\right|_{\left(W_{\infty}^{2}(\Omega)\right)^{2}}: i \in\{1,2, \ldots, s\}, n \in\{1,2, \ldots, N\}\right\} \leq C,
\end{gathered}
$$

and that smallness of the time-continuous solution is ensured on the considered time interval, the global error bound

$$
\left|\mathcal{A}^{1 / 2}\left(\mathbf{u}^{(N)}-\mathbf{u}(T)\right)\right|_{\left(L^{2}(\Omega)\right)^{2}}^{2} \leq C\left(\left|\mathcal{A}^{1 / 2}\left(\mathbf{u}^{(0)}-\mathbf{u}(0)\right)\right|_{\left(L^{2}(\Omega)\right)^{2}}^{2}+\tau_{\max }^{2 r}\right)
$$

holds for $\tau_{\max }=\max \left\{\tau_{n}: n \in\{0,1, \ldots, N-1\}\right\}$ sufficiently small. The arising constant in particular depends on the quantities in (4.10). 
Remark. For the convenience of the reader, we restate the global error estimate for the Westervelt equation with $k=0$ employing Sobolev-norms

$$
\begin{aligned}
& \quad\left|p^{(N)}-p(T)\right|_{W_{2}^{1}(\Omega)}^{2}+\left|\partial_{t} p^{(N)}-\partial_{t} p(T)\right|_{L^{2}(\Omega)}^{2} \\
& \quad \leq C\left(\left|p^{(0)}-p(0)\right|_{W_{2}^{1}(\Omega)}^{2}+\left|\partial_{t} p^{(0)}-\partial_{t} p(0)\right|_{L^{2}(\Omega)}^{2}+\tau_{\max }^{2 r}\right) .
\end{aligned}
$$

4.2. Jordan-Moore-Gibson-Thompson equation. We next consider the Jordan-Moore-GibsonThompson equation (2.2), where the defining nonlinear operator and its Fréchet derivative are given by

$$
\begin{gathered}
\mathbf{v}=\left(v_{0}, v_{1}, v_{2}\right), \quad \mathbf{F}(\mathbf{v})=\left(\begin{array}{c}
v_{1} \\
v_{2} \\
F_{3}(\mathbf{v})
\end{array}\right), \quad \mathbf{F}^{\prime}(\mathbf{v})=\left(\begin{array}{ccc}
0 & I & 0 \\
0 & 0 & I \\
F_{31}^{\prime}(\mathbf{v}) & F_{32}^{\prime}(\mathbf{v}) & F_{33}^{\prime}(\mathbf{v})
\end{array}\right), \\
F_{3}(\mathbf{v})=\frac{1}{T_{\text {rel }}}\left(-\beta_{1} \mathscr{A} v_{0}-\beta_{2} \mathscr{A} v_{1}-\left(1-\beta_{3} v_{0}\right) v_{2}+\beta_{3} v_{1}^{2}\right), \\
F_{31}^{\prime}(\mathbf{v})=f_{1}(\mathbf{v})-\frac{\beta_{1}}{T_{r e l}} \mathscr{A}, \quad F_{32}^{\prime}(\mathbf{v})=f_{2}(\mathbf{v})-\frac{\beta_{2}}{T_{\text {rel }}} \mathscr{A}, \quad F_{33}^{\prime}(\mathbf{v})=f_{3}(\mathbf{v}), \\
f_{1}(\mathbf{v})=\frac{\beta_{3}}{T_{\text {rel }}} v_{2}, \quad f_{2}(\mathbf{v})=\frac{2 \beta_{3}}{T_{\text {rel }}} v_{1}, \quad f_{3}(\mathbf{v})=-\frac{1}{T_{\text {rel }}}\left(1-\beta_{3} v_{0}\right), \\
\beta_{1}=c^{2}, \quad \beta_{2}=b, \quad \beta_{3}=\frac{2 \beta_{a}}{\rho c^{2}} .
\end{gathered}
$$

Due to the fact that the analysis is significantly more involved, we include detailed arguments for the implicit Euler method satisfying

$$
\mathbf{e}^{(n+1)}-\mathbf{e}^{(n)}=\tau_{n} \mathbf{F}^{\prime}\left(\mathbf{u}\left(t_{n+1}\right)+\frac{1}{2} \mathbf{e}^{(n+1)}\right) \mathbf{e}^{(n+1)}+\tau_{n} \mathbf{r}_{1}^{(n)}, \quad n \in\{0,1, \ldots, N-1\} .
$$

We in particular employ the regularity requirements

$$
\begin{gathered}
\max _{\ell \in\{1,2,3\}} \max _{n \in\{1,2, \ldots, N\}}\left|f_{\ell}\left(\mathbf{u}\left(t_{n+1}\right)+\frac{1}{2} \mathbf{e}^{(n+1)}\right)\right|_{L^{\infty}(\Omega)} \leq C_{u}, \\
C_{u}=C_{u}\left(\max _{n \in\{1,2, \ldots, N\}}\left|\mathbf{u}^{(n)}\right|_{\left(L^{\infty}(\Omega)\right)^{3}},\left|u_{0}\right|_{W_{\infty}^{2}\left(0, T ; L^{\infty}(\Omega)\right)}\right) .
\end{gathered}
$$

(i) On the one hand, we test with

$$
\left(\begin{array}{c}
0 \\
\frac{\beta_{2}}{T_{\text {rel }}} \mathscr{A} e_{1}^{(n+1)} \\
e_{2}^{(n+1)}
\end{array}\right)
$$

and perform integration-by-parts. Together with the elementary relation in (3.2), this yields

$$
\begin{aligned}
\mid \mathscr{A}^{1 / 2} & \left.e_{1}^{(n+1)}\right|^{2}-\left|\mathscr{A}^{1 / 2} e_{1}^{(n)}\right|^{2}+\left|e_{2}^{(n+1)}\right|^{2}-\left|e_{2}^{(n)}\right|^{2} \\
\leq & C\left(C_{u}\right) \tau_{n}\left(\left|\mathscr{A} e_{0}^{(n+1)}\right|^{2}+\left|\mathscr{A}^{1 / 2} e_{1}^{(n+1)}\right|^{2}+\left|e_{2}^{(n+1)}\right|^{2}\right) \\
\quad & +C\left(\left|u_{0}\right|_{W_{\infty}^{3}\left(0, T ; W_{2}^{1}(\Omega)\right)}^{2}+\left|u_{0}\right|_{W_{\infty}^{4}\left(0, T ; L^{2}(\Omega)\right)}^{2}\right) \tau_{n}^{3}, \quad n \in\{0,1, \ldots, N-1\},
\end{aligned}
$$

with a generic constant $C>0$ that in particular depends on $C_{u}$. 
(ii) On the other hand, testing with

$$
\left(\begin{array}{c}
0 \\
\mathscr{A} e_{1}^{(n+1)} \\
\mathscr{A} e_{1}^{(n+1)}
\end{array}\right)
$$

and employing smallness of the quantity $C_{u}$, we have

$$
\begin{aligned}
& \left|\mathscr{A}^{1 / 2} e_{1}^{(n+1)}\right|^{2}-\left|\mathscr{A}^{1 / 2} e_{1}^{(n)}\right|^{2}+\tau_{n}\left|\mathscr{A} e_{1}^{(n+1)}\right|^{2} \\
& \leq C \\
& \quad C\left(C_{u}\right) \tau_{n}\left(\left|\mathscr{A} e_{0}^{(n+1)}\right|^{2}+\left|e_{2}^{(n+1)}\right|^{2}\right) \\
& \quad+C\left(\left|u_{0}\right|_{W_{\infty}^{3}\left(0, T ; L^{2}(\Omega)\right)}^{2}+\left|u_{0}\right|_{W_{\infty}^{4}\left(0, T ; L^{2}(\Omega)\right)}^{2}\right) \tau_{n}^{3}, \quad n \in\{0,1, \ldots, N-1\},
\end{aligned}
$$

where the generic constant $C>0$ again in particular depends on $C_{u}$.

(iii) Combining both bounds, summation and a Gronwall inequality show

$$
\begin{gathered}
\left|\mathscr{A}^{1 / 2} e_{1}^{(N)}\right|^{2}+\left|e_{2}^{(N)}\right|^{2} \leq C\left(\left|\mathscr{A}^{1 / 2} e_{1}^{(0)}\right|^{2}+\left|e_{2}^{(n)}\right|^{2}+\tau_{\max }^{2}\right) \\
\tau_{\max }=\max \left\{\tau_{n}: n \in\{0,1, \ldots, N-1\}\right\}
\end{gathered}
$$

More generally, we obtain the following result.

Theorem 4.2 (Jordan-Moore-Gibson-Thompson equation). Let $\mathbf{u}=\left(u_{0}, u_{1}, u_{2}\right)^{T}=\left(u, u^{\prime}, u^{\prime \prime}\right)^{T}$ denote the solution to (2.2), and set $\mathcal{A}=\operatorname{diag}(0, \mathscr{A}, I)$. Suppose that the considered stiffly accurate Runge-Kutta method of nonstiff order $p$ and stage order q fulfills the fundamental condition (3.4). Provided that the time-continuous and time-discrete solutions satisfy the regularity requirements

$$
\begin{gathered}
\left|u_{0}\right|_{W_{\infty}^{2}\left(0, T ; W_{\infty}^{2}(\Omega)\right)}+\left|u_{0}\right|_{W_{\infty}^{r+2}\left(0, T ; W_{2}^{1}(\Omega)\right)}+\left|u_{0}\right|_{W_{\infty}^{r+3}\left(0, T ; L^{2}(\Omega)\right)} \leq C, \quad r=\min \{p, q\} \\
\max \left\{\left|\mathbf{U}_{i}^{(n)}\right|_{\left(W_{\infty}^{2}(\Omega)\right)^{3}}: i \in\{1,2, \ldots, s\}, n \in\{1,2, \ldots, N\}\right\} \leq C
\end{gathered}
$$

and that non-degeneracy is ensured on the considered time interval, the global error bound

$$
\left|\mathcal{A}^{1 / 2}\left(\mathbf{u}^{(N)}-\mathbf{u}(T)\right)\right|_{\left(L^{2}(\Omega)\right)^{3}}^{2} \leq C\left(\left|\mathcal{A}^{1 / 2}\left(\mathbf{u}^{(0)}-\mathbf{u}(0)\right)\right|_{\left(L^{2}(\Omega)\right)^{3}}^{2}+\tau_{\max }^{2 r}\right)
$$

holds for $\tau_{\max }=\max \left\{\tau_{n}: n \in\{0,1, \ldots, N-1\}\right\}$ sufficiently small. The arising constant in particular depends on the quantities in (4.11).

Remark. Employing Sobolev-norms, the stated global error estimate reads as

$$
\begin{aligned}
& \quad\left|\partial_{t} p^{(N)}-\partial_{t} p(T)\right|_{W_{2}^{1}(\Omega)}^{2}+\left|\partial_{t t} p^{(N)}-\partial_{t t} p(T)\right|_{L^{2}(\Omega)}^{2} \\
& \quad \leq C\left(\left|\partial_{t} p^{(0)}-\partial_{t} p(0)\right|_{W_{2}^{1}(\Omega)}^{2}+\left|\partial_{t t} p^{(0)}-\partial_{t t} p(0)\right|_{L^{2}(\Omega)}^{2}+\tau_{\max }^{2 r}\right) .
\end{aligned}
$$


4.3. Blackstock-Crighton-Brunnhuber-Jordan-Kuznetsov equation. For (2.3), similar calculations yield

$$
\begin{aligned}
& \mathbf{v}=\left(v_{0}, v_{1}, v_{2}\right), \quad \mathbf{F}(\mathbf{v})=\left(\begin{array}{c}
v_{1} \\
v_{2} \\
F_{3}(\mathbf{v})
\end{array}\right), \quad \mathbf{F}^{\prime}(\mathbf{v})=\left(\begin{array}{ccc}
0 & I & 0 \\
0 & 0 & I \\
F_{31}^{\prime}(\mathbf{v}) & F_{32}^{\prime}(\mathbf{v}) & F_{33}^{\prime}(\mathbf{v})
\end{array}\right), \\
& F_{3}(\mathbf{v})=-\left(1+\beta_{5} v_{1}\right)^{-1} \\
& \times\left(\beta_{3} \mathscr{A} v_{1}+\beta_{2} \mathscr{A}^{2} v_{1}+\beta_{1} \mathscr{A} v_{2}+\beta_{4} \mathscr{A}^{2} v_{0}+\beta_{5} v_{2}^{2}+2 \beta_{6}\left|\nabla v_{1}\right|^{2}+2 \beta_{6} \nabla v_{0} \cdot \nabla v_{2}\right), \\
& F_{31}^{\prime}(\mathbf{v})=-f_{11}(\mathbf{v}) \mathscr{A}^{2}-f_{12}(\mathbf{v}) \cdot \nabla, \\
& f_{11}(\mathbf{v})= \beta_{4}\left(1+\beta_{5} v_{1}\right)^{-1}, \quad f_{12}(\mathbf{v})=2 \beta_{6}\left(1+\beta_{5} v_{1}\right)^{-1} \nabla v_{2}, \\
& F_{32}^{\prime}(\mathbf{v})= f_{21}(\mathbf{v})-f_{22}(\mathbf{v}) \mathscr{A}^{2}-f_{23}(\mathbf{v}) \mathscr{A}-f_{24}(\mathbf{v}) \cdot \nabla, \\
& f_{21}(\mathbf{v})= \beta_{5}\left(1+\beta_{5} v_{1}\right)^{-2} \\
& \times\left(\beta_{3} \mathscr{A}_{v_{1}}+\beta_{2} \mathscr{A}^{2} v_{1}+\beta_{1} \mathscr{A} v_{2}+\beta_{4} \mathscr{A}^{2} v_{0}+\beta_{5} v_{2}^{2}+2 \beta_{6}\left|\nabla v_{1}\right|^{2}+2 \beta_{6} \nabla v_{0} \cdot \nabla v_{2}\right), \\
& f_{22}(\mathbf{v})= \beta_{2}\left(1+\beta_{5} v_{1}\right)^{-1}, \quad f_{23}(\mathbf{v})=\beta_{3}\left(1+\beta_{5} v_{1}\right)^{-1}, \quad f_{24}(\mathbf{v})=4 \beta_{6}\left(1+\beta_{5} v_{1}\right)^{-1} \nabla v_{1}, \\
& F_{33}^{\prime}(\mathbf{v})=-f_{31}(\mathbf{v})-f_{32}(\mathbf{v}) \mathscr{A}_{-}-f_{33}(\mathbf{v}) \cdot \nabla, \\
& f_{31}(\mathbf{v})= 2 \beta_{5}\left(1+\beta_{5} v_{1}\right)^{-1} v_{2}, \quad f_{32}(\mathbf{v})=\beta_{1}\left(1+\beta_{5} v_{1}\right)^{-1}, \quad f_{33}(\mathbf{v})=2 \beta_{6}\left(1+\beta_{5} v_{1}\right)^{-1} \nabla v_{0},
\end{aligned}
$$

with certain constants $\beta_{1}, \beta_{2}, \beta_{3}, \beta_{4}, \beta_{5}, \beta_{6}>0$. Again, we include detailed calculations for the implicit Euler method

$$
\begin{gathered}
\mathbf{e}^{(n+1)}-\mathbf{e}^{(n)}=\tau_{n} \int_{0}^{1} \mathbf{F}^{\prime}\left(\sigma \mathbf{u}^{(n+1)}+(1-\sigma) \mathbf{u}\left(t_{n+1}\right)\right) \mathrm{d} \sigma \mathbf{e}^{(n+1)}+\tau_{n} \mathbf{r}_{1}^{(n)}, \\
n \in\{0,1, \ldots, N-1\}
\end{gathered}
$$

making use of the regularity requirements

$$
\begin{gathered}
\max _{\ell \in\{11,12,21,22,23,24,31,32,33\}} \max _{n \in\{1,2, \ldots, N\}}\left|\int_{0}^{1} f_{\ell}\left(\sigma \mathbf{u}^{(n+1)}+(1-\sigma) \mathbf{u}\left(t_{n+1}\right)\right) \mathrm{d} \sigma\right|_{L^{\infty}(\Omega)} \leq C_{u}, \\
C_{u}=C_{u}\left(\max _{n \in\{1,2, \ldots, N\}}\left|\mathbf{u}^{(n)}\right|_{\left.\left(W_{\infty}^{4}(\Omega)\right)^{3},\left|u_{0}\right|_{W_{\infty}^{2}\left(0, T ; W_{\infty}^{4}(\Omega)\right)}\right),}\right. \\
\min _{\ell \in\{22,32\}} \underset{x \in \Omega}{\operatorname{ess} \inf _{x \in} \int_{0}^{1} f_{\ell}\left(\sigma \mathbf{u}^{(n+1)}+(1-\sigma) \mathbf{u}\left(t_{n+1}\right)\right)(x) \mathrm{d} \sigma \geq \underline{C_{u}} .}
\end{gathered}
$$

(i) On the one hand, we test with

$$
\left(\begin{array}{c}
0 \\
\mathscr{A}^{3} e_{1}^{(n+1)} \\
\mathscr{A} e_{2}^{(n+1)}
\end{array}\right)
$$


and perform integration-by-parts, which yields

$$
\begin{aligned}
& \left|\mathscr{A}^{3 / 2} e_{1}^{(n+1)}\right|^{2}-\left|\mathscr{A}^{3 / 2} e_{1}^{(n)}\right|^{2}+\left|\mathscr{A}^{1 / 2} e_{2}^{(n+1)}\right|^{2}-\left|\mathscr{A}^{1 / 2} e_{2}^{(n)}\right|^{2}+\tau_{n}\left|\mathscr{A}_{2}^{(n+1)}\right|^{2} \\
& \leq C \\
& \quad \tau_{n}\left(\left|\mathscr{A}^{2} e_{0}^{(n+1)}\right|^{2}+\left|\mathscr{A}^{2} e_{1}^{(n+1)}\right|^{2}\right) \\
& \quad+C\left(\left|u_{0}\right|_{W_{\infty}^{3}\left(0, T ; W_{2}^{2}(\Omega)\right)}+\left|u_{0}\right|_{W_{\infty}^{4}\left(0, T ; L^{2}(\Omega)\right)}\right) \tau_{n}^{3}, \quad n \in\{0,1, \ldots, N-1\},
\end{aligned}
$$

with a generic constant $C>0$.

(ii) On the other hand, testing with

$$
\left(\begin{array}{c}
0 \\
\mathscr{A}^{3} e_{1}^{(n+1)} \\
\mathscr{A}^{2} e_{1}^{(n+1)}
\end{array}\right)
$$

we arrive at the bound

$$
\begin{aligned}
\mid \mathscr{A}^{3 / 2} & \left.e_{1}^{(n+1)}\right|^{2}-\left|\mathscr{A}^{3 / 2} e_{1}^{(n)}\right|^{2}+\tau_{n}\left|\mathscr{A}^{2} e_{1}^{(n+1)}\right|^{2} \\
\leq & C \tau_{n}\left(\left|\mathscr{A}^{2} e_{0}^{(n+1)}\right|^{2}+\left|\mathscr{A} e_{2}^{(n+1)}\right|^{2}\right) \\
& +C\left(\left|u_{0}\right|_{W_{\infty}^{3}\left(0, T ; W_{2}^{2}(\Omega)\right)}+\left|u_{0}\right|_{W_{\infty}^{4}\left(0, T ; L^{2}(\Omega)\right)}\right) \tau_{n}^{3}, \quad n \in\{0,1, \ldots, N-1\} .
\end{aligned}
$$

(iii) Combining both bounds, summation and a Gronwall inequality show

$$
\begin{gathered}
\left|\mathscr{A}^{3 / 2} e_{1}^{(N)}\right|^{2}+\left|\mathscr{A}^{1 / 2} e_{2}^{(N)}\right|^{2} \leq C\left(\left|\mathscr{A}^{3 / 2} e_{1}^{(0)}\right|^{2}+\left|\mathscr{A}^{1 / 2} e_{2}^{(n)}\right|^{2}+\tau_{\max }^{2}\right), \\
\tau_{\max }=\max \left\{\tau_{n}: n \in\{0,1, \ldots, N-1\}\right\} .
\end{gathered}
$$

More generally, we obtain the following result.

Theorem 4.3 (Blackstock-Crighton-Brunnhuber-Jordan-Kuznetsov equation). Let $\mathbf{u}=\left(u_{0}, u_{1}\right.$, $\left.u_{2}\right)^{T}=\left(u, u^{\prime}, u^{\prime \prime}\right)^{T}$ denote the solution to (2.3), and set $\mathcal{A}=\operatorname{diag}\left(0, \mathscr{A}^{3}, \mathscr{A}\right)$. Suppose that the considered stiffly accurate Runge-Kutta method of nonstiff order $p$ and stage order $q$ fulfills the fundamental condition (3.4). Provided that the time-continuous and time-discrete solutions satisfy the regularity requirements

$$
\begin{gathered}
\left|u_{0}\right|_{W_{\infty}^{2}\left(0, T ; W_{\infty}^{4}(\Omega)\right)}+\left|u_{0}\right|_{W_{\infty}^{r+2}\left(0, T ; W_{2}^{2}(\Omega)\right)}+\left|u_{0}\right|_{W_{\infty}^{r+3}\left(0, T ; L^{2}(\Omega)\right)} \leq C, \quad r=\min \{p, q\}, \\
\max \left\{\left|\mathbf{U}_{i}^{(n)}\right|_{\left(W_{\infty}^{4}(\Omega)\right)^{3}}: i \in\{1,2, \ldots, s\}, n \in\{1,2, \ldots, N\}\right\} \leq C,
\end{gathered}
$$

and that non-degeneracy is ensured on the considered time interval, the global error bound

$$
\left|\mathcal{A}^{1 / 2}\left(\mathbf{u}^{(N)}-\mathbf{u}(T)\right)\right|_{\left(L^{2}(\Omega)\right)^{3}}^{2} \leq C\left(\left|\mathcal{A}^{1 / 2}\left(\mathbf{u}^{(0)}-\mathbf{u}(0)\right)\right|_{\left(L^{2}(\Omega)\right)^{3}}^{2}+\tau_{\max }^{2 r}\right)
$$

holds for $\tau_{\max }=\max \left\{\tau_{n}: n \in\{0,1, \ldots, N-1\}\right\}$ sufficiently small. The arising constant in particular depends on the quantities in (4.12).

Remark. Employing Sobolev-norms, the stated global error estimate reads as

$$
\begin{aligned}
& \left|\partial_{t} \psi^{(N)}-\partial_{t} \psi(T)\right|_{W_{2}^{3}(\Omega)}^{2}+\left|\partial_{t t} \psi^{(N)}-\partial_{t t} \psi(T)\right|_{W_{2}^{1}(\Omega)}^{2} \\
& \quad \leq C\left(\left|\partial_{t} \psi^{(0)}-\partial_{t} \psi(0)\right|_{W_{2}^{3}(\Omega)}^{2}+\left|\partial_{t t} \psi^{(0)}-\partial_{t t} \psi(0)\right|_{W_{2}^{1}(\Omega)}^{2}+\tau_{\max }^{2 r}\right) .
\end{aligned}
$$




\section{ENERGY EESTIMATES}

In this section, we deduce uniform energy estimates for the three nonlinear acoustic equations dicussed in the previous sections on a more abstract level, thus potentially comprising other nonlinear evolution equations. The purpose of these energy estimates, that in parts also differ from the energy estimates in the literature on nonlinear acoustics so far, is their transfer to time discretisation by stiffly accurate Runge Kutta methods.

Abstract higher order PDE model. As already indicated in (2.1b), (2.2b), (2.3b), we can write the equations under consideration in the framework on the abstract higher order PDE model

$$
\begin{aligned}
& \partial_{t}^{m+1} u+\mathrm{b}\left(u, \partial_{t} u, \ldots, \partial_{t}^{m} u\right) \mathscr{A}^{k_{m}} \partial_{t}^{m} u+\sum_{i=1}^{r} \mathrm{c}_{i}\left(u, \partial_{t} u, \ldots, \partial_{t}^{m} u\right) \mathscr{A}^{\ell_{i}} \partial_{t}^{m-1} u \\
& =\mathscr{B}\left(u, \partial_{t} u, \ldots, \partial_{t}^{m} u\right)\left[\left(u, \partial_{t} u, \ldots, \partial_{t}^{m} u\right)\right]
\end{aligned}
$$

with $0 \leq \ell_{1}<\ell_{2}<\cdots \ell_{r}, 0 \leq k_{m}$,

$$
\mathscr{B}: \mathscr{D}_{1}(\mathscr{B}) \rightarrow L\left(\mathscr{D}_{2}(\mathscr{B}), \mathscr{H}\right), \quad \mathscr{D}_{1}(\mathscr{B}), \mathscr{D}_{2}(\mathscr{B}) \subseteq \mathscr{H}^{m+1}
$$

and a selfadjoint densely defined operator $\mathscr{A}$ on a Hilbert space $\mathscr{H}$

$$
\mathscr{A}: \mathscr{D}(\mathscr{A}) \rightarrow \mathscr{H}, \quad \mathscr{D} \subseteq \mathscr{H}
$$

satisfying a Poincaré-Friedrichs type inequality

$$
|v| \leq C_{P F}|\mathscr{A} v|, \quad v \in \mathscr{D}(\mathscr{A})
$$

(e.g. $\mathscr{A}=-\Delta_{D}, \mathscr{D}(\mathscr{A})=H^{2}(\Omega) \cap H_{0}^{1}(\Omega), \mathscr{H}=L^{2}(\Omega)$ );

We will use the notation $|\cdot|$ and $(\cdot \mid \cdot)$ for norm and inner product on $\mathscr{H}$, respectively.

Recall that this applies to the above models with the following settings:

- Westervelt: $m=1, k_{m}=1, r=1, \ell_{r}=1$.

- JMGT: $m=2, k_{m}=0, r=1, \ell_{r}=1$.

- BCBJK: $m=2, k_{m}=1, r=2, \ell_{1}=1, \ell_{r}=2$.

First order reformulation. We rewrite (5.1) as

$$
\begin{gathered}
\mathbf{u}^{\prime}(t)+\mathbf{A}(\mathbf{u}(t)) \mathbf{u}(t)=\mathbf{B}(\mathbf{u}(t))[\mathbf{u}(t)], \quad t \in(0, T) \\
\mathbf{u}(t)=\left(\begin{array}{c}
u_{0}(t) \\
u_{1}(t) \\
\vdots \\
u_{m}(t)
\end{array}\right), \quad \mathbf{B}(\mathbf{u}(t))[\mathbf{u}(t)]=\left(\begin{array}{c}
0 \\
\vdots \\
0 \\
\mathscr{B}(\mathbf{u}(t))[\mathbf{u}(t)]
\end{array}\right), \\
\mathbf{A}(\mathbf{u}(t))=\left(\begin{array}{cccccc}
0 & -I & 0 & \ldots & \ldots & 0 \\
\vdots & 0 & -I & 0 & \ldots & \vdots \\
\vdots & & \ddots & \ddots & 0 & \vdots \\
\vdots & & & \ddots & -I & 0 \\
0 & \ldots & \ldots & \ldots & \sum_{i=1}^{r} \mathrm{c}_{i}(\mathbf{u}(t)) \mathscr{A}^{\ell_{i}} & \mathrm{~b}(\mathbf{u}(t)) \mathscr{A}^{k_{m}}
\end{array}\right) .
\end{gathered}
$$


5.1. Energy estimates in the linearised setting. With coefficients $\mathrm{b}, \mathrm{c}_{i} \in L^{\infty}(0, T ; L(\mathscr{H}, \mathscr{H}))$ (i.e., in case of $\mathscr{H}=L^{2}(\Omega)$ simply b,$\left.c_{i} \in L^{\infty}\left(0, T ; L^{\infty}(\Omega)\right)\right)$ ) that may depend on space and time and a one-homogeneosly bounded operator $\mathscr{G}$

$$
\begin{aligned}
& \mathscr{G}:(0, T) \times X_{0} \rightarrow \mathscr{H}, \quad X_{0}=X_{0,0} \times X_{0,1} \times X_{0, m} \subseteq \mathscr{H}^{m+1} \\
& |\mathscr{G}(t)[\vec{v}]|=\left|\mathscr{G}\left(t ; v_{0}, \ldots, v_{m}\right)\right| \leq C_{G}\|\vec{v}\|_{X_{0}}
\end{aligned}
$$

with a uniform constant $C_{G}$ (note that $\mathscr{G}$ does not necessarily need to be linear to satisfy this, but it will be in our application of the estimates to the models above) with the right space $X_{0}$ yet to be determined, and $f \in L^{2}(0, T ; \mathscr{H})$, we consider

$$
\partial_{t}^{m+1} u+\mathrm{b} \mathscr{A}^{k_{m}} \partial_{t}^{m} u+\sum_{i=1}^{r} \mathrm{c}_{i} \mathscr{A}^{\ell_{i}} \partial_{t}^{m-1} u=\mathscr{G}\left[u, \partial_{t} u, \ldots, \partial_{t}^{m} u\right]+f
$$

i.e., written as a first order system

$$
\begin{gathered}
\mathbf{u}^{\prime}(t)+\mathbf{A}(t) \mathbf{u}(t)=(0, \cdots, 0, \mathscr{G}(t)[\mathbf{u}(t)]+f(t))^{T} \\
\text { with } \mathbf{A}=\left(\begin{array}{ccccc}
0 & -I & 0 & \ldots & 0 \\
0 & 0 & -I & 0 & \cdots \\
\vdots & \ddots & \ddots & \ddots \\
0 & & & \sum_{i=1}^{r} c_{i} \mathscr{A}^{\ell_{i}} & \mathrm{~b} \mathscr{A}^{k_{m}}
\end{array}\right) .
\end{gathered}
$$

First of all we demonstrate the ideas with constants $b>0, c_{i} \geq 0$.

Testing with

$$
\left(0, \ldots, 0, \sum_{i=1}^{r} \mathscr{A}^{k_{m}}\left[\mathrm{c}_{i} \mathscr{A}^{\ell_{i}} u_{m-1}(t)\right], \mathscr{A}^{k_{m}} u_{m}(t)\right)^{T}
$$

yields

$$
\begin{aligned}
& \left(u_{m-1}^{\prime}(t)-u_{m}(t) \mid \sum_{i=1}^{r} \mathscr{A}^{k_{m}}\left[\mathrm{c}_{i} \mathscr{A}^{\ell_{i}} u_{m-1}(t)\right]\right) \\
& \left.\quad+\left(u_{m}^{\prime}(t)+\sum_{i=1}^{r} \mathrm{c}_{i} \mathscr{A}^{\ell_{i}} u_{m-1}(t)\right]+\mathrm{b} \mathscr{A}^{k_{m}} u_{m}(t) \mid \mathscr{A}^{k_{m}} u_{m}(t)\right) \\
& =\frac{1}{2} \frac{d}{d t}\left|\mathscr{A}^{k_{m} / 2} u_{m}\right|^{2}(t)+\sum_{i=1}^{r} \frac{\mathrm{c}_{i}}{2} \frac{d}{d t}\left|\mathscr{A}^{\left(k_{m}+\ell_{i}\right) / 2} u_{m-1}\right|^{2}(t)+\mathrm{b}\left|\mathscr{A}^{k_{m}} u_{m}(t)\right|^{2} \\
& =\left(\mathscr{G}(t)[\mathbf{u}(t)]+f \mid \mathscr{A}^{k_{m}} u_{m}(t)\right) \leq \frac{1}{\mathrm{~b}}|\mathscr{G}(t)[\mathbf{u}(t)]|^{2}+\frac{1}{\mathrm{~b}}|f(t)|^{2}+\frac{\mathrm{b}}{2}\left|\mathscr{A}^{k_{m}} u_{m}(t)\right|^{2},
\end{aligned}
$$

hence after integration and taking the supremum wrt. time, we get, for arbitrary $s \in[0, T]$, using $\sup _{t \in(0, s)}(a(t)+b(t)+c(t)) \geq \frac{1}{2} \sup _{t \in(0, T)} a(t)+\frac{1}{4} \sup _{t \in(0, T)} b(t)+\frac{1}{4} \sup _{t \in(0, T)} c(t)$,

$$
\begin{aligned}
& \left\|\mathscr{A}^{k_{m} / 2} u_{m}\right\|_{L^{\infty}(0, s ; \mathscr{H})}^{2}+\sum_{i=1}^{r} \frac{\mathrm{c}_{i}}{2}\left\|\mathscr{A}^{\left(k_{m}+\ell_{i}\right) / 2} u_{m-1}\right\|_{L^{\infty}(0, T ; \mathscr{H})}^{2}+\frac{\mathrm{b}}{2}\left\|\mathscr{A}^{k_{m}} u_{m}(t)\right\|_{L^{2}(0, s ; \mathscr{H})}^{2} \\
& \leq \frac{4}{\mathrm{~b}}\left(\|\mathscr{G}[\mathbf{u}]\|_{L^{2}(0, s ; \mathscr{H})}^{2}+\|f\|_{L^{2}(0, s ; \mathscr{H})}^{2}\right)+\left|\mathscr{A}^{k_{m} / 2} u_{m}(0)\right|^{2}+\sum_{i=1}^{r} \frac{\mathrm{c}_{i}}{2}\left|\mathscr{A}^{\left(k_{m}+\ell_{i}\right) / 2} u_{m-1}(0)\right|^{2},
\end{aligned}
$$

where due to the fact that $u_{m-2}(t)=u_{m-2}(0)+\int_{0}^{t} u_{m-1}(\tau) d \tau$, with

$$
\overline{\mathbf{u}}(t):=\left(u_{0}(t), \ldots, u_{m-3}(t), u_{m-2}(0)+\int_{0}^{t} u_{m-1}(\tau) d \tau, u_{m-1}(t), u_{m}(t)\right)
$$


we have

$$
\begin{aligned}
& \|\mathscr{G}[\mathbf{u}]\|_{L^{2}(0, s ; \mathscr{H})}^{2} \leq C_{G}^{2} \int_{0}^{s}\|\overline{\mathbf{u}}(t)\|_{X_{0}}^{2} d t \\
& \leq C_{G}^{2} \int_{0}^{s}\left(\|\mathbf{u}(t)\|_{X_{0}}+\left\|u_{m-2}(0)\right\|_{X_{0, m-2}}+\int_{0}^{t}\left\|u_{m-1}(\tau)\right\|_{X_{0, m-2}} d \tau\right) d t .
\end{aligned}
$$

This extends to the case of space and time dependent multipliers

$$
\begin{aligned}
& \mathrm{b}, \mathrm{c}_{i} \in L^{\infty}(0, T ; L(\mathscr{H}, \mathscr{H})), \quad \mathrm{b} \geq \underline{\mathrm{b}}>0 \quad \mathrm{c}_{i} \geq \underline{\mathrm{c}}_{i}>0 \\
& \text { and either } \mathrm{b}^{\prime}, \mathrm{c}_{i}^{\prime} \in L^{\infty}(0, T ; L(\mathscr{H}, \mathscr{H})) \\
& \quad \text { or }\left\|\mathrm{b}^{\prime}\right\|_{L^{1}(0, T ; L(\mathscr{H}, \mathscr{H}))},\left\|\mathrm{c}_{i}^{\prime}\right\|_{L^{1}(0, T ; L(\mathscr{H}, \mathscr{H}))} \text { small enough }
\end{aligned}
$$

via the identity

$$
\begin{aligned}
& \left.\frac{1}{2} \frac{d}{d t}\left|\sqrt{\mathrm{c}} \mathscr{A}^{(p+q) / 2} v\right|^{2}(t)=\frac{1}{2} \frac{d}{d t}\left(\mathscr{A}^{(p+q) / 2} v \mid \mathrm{c} \mathscr{A}^{(p+q) / 2} v\right)(t)\right) \\
& =\left(\mathscr{A}^{(p+q) / 2} v^{\prime}(t) \mid \mathrm{c}(t) \mathscr{A}^{(p+q) / 2} v(t)\right)+\frac{1}{2}\left(\mathscr{A}^{(p+q) / 2} v(t) \mid \mathrm{c}^{\prime}(t) \mathscr{A}^{(p+q) / 2} v(t)\right) \\
& =\left(v^{\prime}(t) \mid \mathscr{A}^{p}\left[\mathrm{c}(t) \mathscr{A}^{q} v(t)\right]\right) \\
& \quad+\left(v^{\prime}(t) \mid \mathscr{A}^{(p+q) / 2}\left[\mathrm{c}(t) \mathscr{A}^{(p+q) / 2} v(t)\right]-\mathscr{A}^{p}\left[\mathrm{c}(t) \mathscr{A}^{q} v(t)\right]\right) \\
& \quad+\frac{1}{2}\left(\mathrm{c}^{\prime}(t) \mathscr{A}^{(p+q) / 2} v(t) \mid \mathscr{A}^{(p+q) / 2} v(t)\right)
\end{aligned}
$$

which in case of $v=u_{m-1}$ and $\mathrm{c}=\mathrm{c}_{i}, p=k_{m}, q=\ell_{i}$, using the fact that $u_{m-1}^{\prime}=u_{m}$, reads as

$$
\begin{aligned}
\frac{1}{2} \frac{d}{d t} \mid & \left.\sqrt{\mathrm{c}} \mathscr{A}^{k_{m}+\ell_{i}} u_{m-1}\right|^{2}(t)=\left(u_{m-1}^{\prime}(t) \mid \mathscr{A}^{k_{m}}\left[\mathrm{c}(t) \mathscr{A}^{\ell_{i}} u_{m-1}(t)\right]\right) \\
& +\left(\mathscr{A}^{k_{m}} u_{m}(t) \mid \mathscr{A}^{\left(\ell_{i}-k_{m}\right) / 2}\left[\mathrm{c}(t) \mathscr{A}^{\left(k_{m}+\ell_{i}\right) / 2} u_{m-1}(t)\right]-\left[\mathrm{c}(t) \mathscr{A}^{\ell_{i}} u_{m-1}(t)\right]\right) \\
& +\frac{1}{2}\left(\mathrm{c}^{\prime}(t) \mathscr{A}^{\left(k_{m}+\ell_{i}\right) / 2} u_{m-1}(t) \mid \mathscr{A}^{\left(k_{m}+\ell_{i}\right) / 2} u_{m-1}(t)\right) .
\end{aligned}
$$

This results in

$$
\begin{aligned}
\frac{1}{2} \frac{d}{d t}\left|\mathscr{A}^{k_{m} / 2} u_{m}\right|^{2}(t)+\frac{1}{2} \sum_{i=1}^{r} \frac{d}{d t}\left|\sqrt{\mathrm{c}_{i}} \mathscr{A}^{\left(k_{m}+\ell_{i}\right) / 2} u_{m-1}\right|^{2}(t)+\left|\sqrt{\mathrm{b}(t)} \mathscr{A}^{k_{m}} u_{m}(t)\right|^{2} \\
=\left(\mathscr{G}(t)[\mathbf{u}(t)]+f(t) \mid \mathscr{A}^{k_{m}} u_{m}(t)\right) \\
\quad+\frac{1}{2} \sum_{i=1}^{r}\left(c_{i}^{\prime}(t) \mathscr{A}^{\left(k_{m}+\ell_{i}\right) / 2} u_{m-1}(t) \mid \mathscr{A}^{\left(k_{m}+\ell_{i}\right) / 2} u_{m-1}(t)\right) \\
+\sum_{i=1}^{r}\left(u_{m-1}^{\prime}(t) \mid \mathscr{A}^{\left(k_{m}+\ell_{i}\right) / 2}\left[c_{i} \mathscr{A}^{\left(k_{m}+\ell_{i}\right) / 2} u_{m-1}(t)\right]-\mathscr{A}^{k_{m}}\left[c_{i} \mathscr{A}^{\ell_{i}} u_{m-1}(t)\right]\right)
\end{aligned}
$$




$$
\begin{aligned}
\leq \underline{\frac{1}{\mathrm{~b}}} \mid \mathscr{G}(t) & {[\mathbf{u}(t)]+\left.f(t)\right|^{2}+\frac{1}{4}\left|\sqrt{\mathrm{b}(t)} \mathscr{A}^{k_{m}} u_{m}(t)\right|^{2} } \\
+ & \frac{1}{2} \sum_{i=1}^{r} \frac{1}{\mathrm{c}_{i}}\left|\mathrm{c}_{i}^{\prime}(t)\right|_{L(\mathscr{H}, \mathscr{H})}\left|\sqrt{\mathrm{c}_{i}(t)} \mathscr{A}^{\left(k_{m}+\ell_{i}\right) / 2} u_{m-1}(t)\right|^{2} \\
+ & \frac{1}{4}\left|\sqrt{\mathrm{b}(t)} \mathscr{A}^{k_{m}} u_{m}(t)\right|^{2} \\
& +\frac{1}{\underline{\mathrm{b}}} \sum_{i=1}^{r}\left|\mathscr{A}^{\left(\ell_{i}-k_{m}\right) / 2}\left[\mathrm{c}_{i} \mathscr{A}^{\left(k_{m}+\ell_{i}\right) / 2} u_{m-1}(t)\right]-\left[\mathrm{c}_{i} \mathscr{A}^{\ell_{i}} u_{m-1}(t)\right]\right|^{2}
\end{aligned}
$$

from which, as above, by time integration an energy estimate can be obtained.

A second energy estimate can be obtained by testing with

$$
\left(0, \ldots, 0, \mathscr{A}^{k_{m}}\left[\mathrm{~b} \mathscr{A}^{\ell_{r}} u_{m-1}(t)\right], \mathscr{A}^{\ell_{r}} u_{m-1}(t)\right)^{T},
$$

which, by using (5.12) with $\mathrm{c}=\mathrm{b}, p=k_{m}, q=\ell_{r}$ yields

$$
\begin{aligned}
& \frac{1}{2} \frac{d}{d t}\left|\sqrt{\mathrm{b}} \mathscr{A}^{\left(k_{m}+\ell_{r}\right) / 2} u_{m-1}\right|^{2}(t)+\sum_{i=1}^{r}\left|\sqrt{\mathrm{c}_{i}} \mathscr{A}^{\left(\ell_{r}+\ell_{i}\right) / 2} u_{m-1}(t)\right|^{2} \\
& =\left(\mathscr{G}(t)[\mathbf{u}(t)]+f(t) \mid \mathscr{A}^{\ell_{r}} u_{m-1}(t)\right) \\
& +\frac{1}{2}\left(\mathrm{~b}^{\prime}(t) \mathscr{A}^{\left(k_{m}+\ell_{r}\right) / 2} u_{m-1}(t) \mid \mathscr{A}^{\left(k_{m}+\ell_{r}\right) / 2} u_{m-1}(t)\right) \\
& +\left(u_{m-1}^{\prime} \mid \mathscr{A}^{\left(k_{m}+\ell_{r}\right) / 2}\left[\mathrm{~b} \mathscr{A}^{\left(k_{m}+\ell_{r}\right) / 2} u_{m-1}(t)\right]-\mathscr{A}^{k_{m}}\left[\mathrm{~b} \mathscr{A}^{\ell_{r}} u_{m-1}(t)\right]\right) \\
& +\sum_{i=1}^{r}\left(u_{m-1} \mid \mathscr{A}^{\left(\ell_{r}+\ell_{i}\right) / 2}\left[\mathrm{c}_{i} \mathscr{A}^{\left(\ell_{r}+\ell_{i}\right) / 2} u_{m-1}(t)\right]-\mathscr{A}^{\ell_{r}}\left[\mathrm{c}_{i} \mathscr{A}^{\ell_{i}} u_{m-1}(t)\right]\right) \\
& \leq \frac{1}{\underline{\mathrm{c}}_{r}}|\mathscr{G}(t)[\mathbf{u}(t)]+f(t)|^{2}+\frac{1}{4}\left|\sqrt{\mathrm{c}_{r}} \mathscr{A}^{\ell_{r}} u_{m-1}(t)\right|^{2}
\end{aligned}
$$

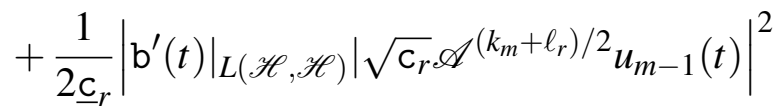

$$
\begin{aligned}
& +\frac{1}{2}\left|\sqrt{\mathrm{b}} \mathscr{A}^{k_{m}} u_{m}(t)\right|^{2}+\frac{1}{2 \underline{\mathrm{b}}}\left|\mathscr{A}^{\left(\ell_{r}-k_{m}\right) / 2}\left[\mathrm{~b} \mathscr{A}^{\left(k_{m}+\ell_{r}\right) / 2} u_{m-1}(t)\right]-\left[\mathrm{b} \mathscr{A}^{\ell_{r}} u_{m-1}(t)\right]\right|^{2} \\
& +\frac{1}{4}\left|\sqrt{\mathrm{c}_{r}} \mathscr{A}^{\ell_{r}} u_{m-1}(t)\right|^{2} \\
& +\frac{1}{\underline{\mathrm{c}}_{r}}\left(\sum_{i=1}^{r}\left|\mathscr{A}^{\left(\ell_{i}-\ell_{r}\right) / 2}\left[\mathrm{c}_{i} \mathscr{A}^{\left(\ell_{r}+\ell_{i}\right) / 2} u_{m-1}(t)\right]-\left[\mathrm{c}_{i} \mathscr{A}^{\ell_{i}} u_{m-1}(t)\right]\right|\right)^{2} .
\end{aligned}
$$

Therewith considering all left hand side terms in (5.13), (5.15), we expect - after time integration - to obtain bounds on

$$
\begin{aligned}
& \left\|\mathscr{A}^{k_{m} / 2} u_{m}\right\|_{L^{\infty}(0, T ; \mathscr{H})}, \quad\left\|\mathscr{A}^{k_{m}} u_{m}\right\|_{L^{2}(0, T ; \mathscr{H})}, \\
& \left\|\mathscr{A}^{k_{m}} u_{m-1}\right\|_{L^{\infty}(0, T ; \mathscr{H})}, \quad\left\|\mathscr{A}^{\left(k_{m}+\ell_{r}\right) / 2} u_{m-1}\right\|_{L^{\infty}(0, T ; \mathscr{H})}, \quad\left\|\mathscr{A}^{\ell_{r}} u_{m-1}\right\|_{L^{2}(0, T ; \mathscr{H})}, \\
& \left\|\mathscr{A}^{\left(k_{m}+\ell_{r}\right) / 2} u_{m-2}\right\|_{L^{\infty}(0, T ; \mathscr{H})}, \quad\left\|\mathscr{A}^{\ell_{r}} u_{m-2}\right\|_{L^{\infty}(0, T ; \mathscr{H})},
\end{aligned}
$$


and therefore, via the identity $u_{j}^{\prime}=u_{j+1}$ and the estimate

$$
\left\|u_{j}\right\|_{L^{\infty}(0, T ; Z)} \leq\left\|u_{j}(0)\right\|_{Z}+\sqrt{T}\left\|u_{j+1}\right\|_{L^{2}(0, T ; Z)},
$$

which allows to inherit the regularity of higher time derivatives,

$$
\begin{aligned}
& \left\|\mathscr{A}^{k_{m} / 2} u_{m}\right\|_{L^{\infty}(0, T ; \mathscr{H})}, \quad\left\|\mathscr{A}^{k_{m}} u_{m}\right\|_{L^{2}(0, T ; \mathscr{H})}, \\
& \left\|\mathscr{A}^{\max \left\{k_{m},\left(k_{m}+\ell_{r}\right) / 2\right\}} u_{m-1}\right\|_{L^{\infty}(0, T ; \mathscr{H})}, \quad\left\|\mathscr{A}^{\max \left\{k_{m}, \ell_{r}\right\}} u_{m-1}\right\|_{L^{2}(0, T ; \mathscr{H})}, \\
& \left\|\mathscr{A}^{k_{m}} u_{m-1}^{\prime}\right\|_{L^{2}(0, T ; \mathscr{H})}, \\
& \left\|\mathscr{A}^{\max \left\{k_{m}, \ell_{r}\right\}} u_{j}\right\|_{L^{\infty}(0, T ; \mathscr{H})}, \quad\left\|\mathscr{A}^{\max \left\{k_{m}, \ell_{r}\right\}} u_{j}^{\prime}\right\|_{L^{2}(0, T ; \mathscr{H})}, \quad j \in\{0, \ldots, m-2\} .
\end{aligned}
$$

Thus we define the energy induced spaces by

$$
\begin{aligned}
X= & \left(L^{\infty}\left(0, T ; \mathscr{D}\left(\mathscr{A}^{\max \left\{k_{m}, \ell_{r}\right\}}\right)\right) \cap H^{1}\left(0, T ; \mathscr{D}\left(\mathscr{A}^{\max \left\{k_{m}, \ell_{r}\right\}}\right)\right)\right)^{m-1} \\
& \times L^{2}\left(0, T ; \mathscr{D}\left(\mathscr{A}^{\max \left\{k_{m}, \ell_{r}\right\}}\right)\right) \cap L^{\infty}\left(0, T ; \mathscr{D}\left(\mathscr{A}^{\left.\max \left\{k_{m}, k_{m}+\ell_{r}\right) / 2\right\}}\right)\right) \\
& \cap H^{1}\left(0, T ; \mathscr{D}\left(\mathscr{A}^{k_{m}}\right)\right) \\
& \times L^{2}\left(0, T ; \mathscr{D}\left(\mathscr{A}^{k_{m}}\right)\right) \cap L^{\infty}\left(0, T ; \mathscr{D}\left(\mathscr{A}^{k_{m} / 2}\right)\right) \\
\subseteq & L^{\infty}\left(0, T ; X_{0}\right) \cap L^{2}\left(0, T ; X_{1}\right)
\end{aligned}
$$

with

$$
\begin{aligned}
X_{0} & =X_{0,0} \times X_{0,1} \times \cdots \times X_{0, m} \\
& =\mathscr{D}\left(\mathscr{A}^{\max \left\{k_{m}, \ell_{r}\right\}}\right)^{m-1} \times \mathscr{D}\left(\mathscr{A}^{\max \left\{k_{m},\left(k_{m}+\ell_{r}\right) / 2\right\}}\right) \times \mathscr{D}\left(\mathscr{A}^{k_{m} / 2}\right) \\
X_{1} & =X_{1,0} \times X_{1,1} \times \cdots \times X_{1, m} \\
& =\mathscr{D}\left(\mathscr{A}^{\max \left\{k_{m}, \ell_{r}\right\}}\right)^{m} \times \mathscr{D}\left(\mathscr{A}^{k_{m}}\right) .
\end{aligned}
$$

Indeed, integrating with respect to time and applying Gronwall's inequality

$$
\begin{aligned}
& \eta(t) \leq a(t)+\int_{0}^{t} b(s) \eta(s) d s \quad \text { for all } t \in(0, T) \\
& \quad \Rightarrow \eta(t) \leq a(t)+\int_{0}^{t} a(s) b(s) \exp \left(\int_{s}^{t} b(\sigma) d \sigma\right) d s \quad \text { for all } t \in(0, T)
\end{aligned}
$$

for $\eta, a, b \geq 0$ (see, e.g, [34, Lemma 2.7]) to the energy estimates resulting from (5.13), (5.15), together with (5.2), $u_{j}(t)=u_{j}(0)+\int_{0}^{t} u_{j+1}(\tau) d \tau$, as well as (5.10) and the fact that $X_{1, m-1}$ continuosly embeds into $X_{0, m-2}$, yields uniform bounds on the energy terms (5.16), provided the initial data satisfy the regularity

$$
\left(u_{0}(0), u_{1}(0), \ldots, u_{m}(0)\right) \in X_{0}
$$

and the terms emerging from space dependence of the multipliers can be dominated by the corresponding energy terms:

$$
\begin{aligned}
& \left|\mathscr{A}^{\left(\ell_{i}-k_{m}\right) / 2}\left[\mathrm{c}_{i} \mathscr{A}^{\left(k_{m}+\ell_{i}\right) / 2} u_{m-1}(t)\right]-\left[\mathrm{c}_{i} \mathscr{A}^{\ell_{i}} u_{m-1}(t)\right]\right| \\
& +\left|\mathscr{A}^{\left(\ell_{r}-k_{m}\right) / 2}\left[\mathrm{~b} \mathscr{A}^{\left(k_{m}+\ell_{r}\right) / 2} u_{m-1}(t)\right]-\left[\mathrm{b} \mathscr{A}^{\ell_{r}} u_{m-1}(t)\right]\right| \\
& +\left|\mathscr{A}^{\left(\ell_{i}-\ell_{r}\right) / 2}\left[\mathrm{c}_{i} \mathscr{A}^{\left(\ell_{r}+\ell_{i}\right) / 2} u_{m-1}(t)\right]-\left[\mathrm{c}_{i} \mathscr{A}^{\ell_{i}} u_{m-1}(t)\right]\right| \\
& \leq C_{\mathrm{bc}}\left(\left|\mathscr{A}^{k_{m}} u_{m-1}(t)\right|+\left|\mathscr{A}^{\left(k_{m}+\ell_{r}\right) / 2} u_{m-1}(t)\right|\right)+c_{\mathrm{bc}}\left|\mathscr{A}^{\ell_{r}} u_{m-1}(t)\right| .
\end{aligned}
$$


We therefore arrive at the following result.

Proposition 5.1. Under conditions (5.2), (5.4), (5.11), (5.20), (5.21), with $c_{\mathrm{bc}}$ small enough, any solution u to (5.5) satisfies the estimate

$$
\left\|\left(u, \partial_{t} u, \ldots, \partial_{t}^{m} u\right)\right\|_{X} \leq C\left(\left\|\left(u(0), \partial_{t} u(0), \ldots, \partial_{t}^{m} u(0)\right)\right\|_{X_{0}}+\|f\|_{L^{2}(0, T ; \mathscr{C})}\right)
$$

for some $C$ depending only on $T$ and the constants $\underline{b b}, \underline{c c_{i}}, C_{P F}, C_{\mathrm{bc}}, C_{G}$.

Remark 5.1. Existence of a solution to (5.5) with given initial data of the regularity prescribed in Proposition 5.1 can be proven, e.g., by means of a Galerkin discretisation with eigenfunctions of $\mathscr{A}$ and taking limits as the discretisation gets finer, based on energy estimates like those in Proposition 5.1, cf., e.g., [21]. Uniqueness follows from Proposition 5.1, since the difference between any two solutions satisfies (5.5) with homogeneous initial data and $f=0$.

5.2. Energy estimates and well-posendess in the nonlinear setting. To establish well-posedness and energy estimates for the nonlinear equation (5.1), i.e., (5.3) with initial conditions

$$
\mathbf{u}(0)=\left(u(0), \partial_{t} u(0), \ldots, \partial_{t}^{m} u(0)\right)=\vec{u}^{0} \in X_{0}
$$

we define the fixed point operator $\mathscr{T}: B_{R}^{X}(0) \rightarrow X$ by assigning to $\mathbf{v} \in B_{R}^{X}(0)=\left\{\mathbf{v} \in X:\|\mathbf{v}\|_{X} \leq\right.$ $R\}$ the solution of the linear initial value problem (5.5), i.e., (5.6) with

$$
\mathrm{b}(t)=\mathrm{b}(\mathbf{v}(t)), \quad \mathrm{c}_{i}(t)=\mathrm{c}_{i}(\mathbf{v}(t)), \quad \mathscr{G}(t)[\vec{v}]=\mathscr{B}(\mathbf{v}(t))[\vec{v}], \quad f=0
$$

and (5.22).

We assume that we can choose $R>0$ such that

$$
B_{R}^{X}(0) \subseteq\left\{\mathbf{v} \in X: \mathrm{b}=\mathrm{b}(\mathbf{v}), \quad \mathrm{c}_{i}=\mathrm{c}_{i}(\mathbf{v}), \quad \mathscr{G}=\mathscr{B}(\mathbf{v}) \text { satisfy }(5.4),(5.11),(5.21)\right\}
$$

(we will verfiy this for the nonlinear acoustics models from Section 2 in Section 5.3 below). Proposition 5.1 then implies that $\mathscr{T}$ is a self-mapping on $B_{R}^{X}(0)$ provided $\left\|\vec{u}_{0}\right\|_{X_{0}} \leq \frac{R}{C}$.

To obtain contractivity of $\mathscr{T}$ we assume that

$$
\begin{aligned}
& \sum_{i=1}^{r}\left\|\mathrm{c}_{i}(\vec{v})-\mathrm{c}_{i}(\overrightarrow{\tilde{v}})\right\|_{L(\mathscr{H}, \mathscr{H})} C_{P F}^{r-i} \leq C_{\mathrm{c}}\|\vec{v}-\overrightarrow{\tilde{v}}\|_{X_{0}}, \quad\|\mathrm{~b}(\vec{v})-\mathrm{b}(\overrightarrow{\tilde{v}})\|_{L(\mathscr{H}, \mathscr{H})} \leq C_{\mathrm{b}}\|\vec{v}-\overrightarrow{\tilde{v}}\|_{X_{0}} \\
& \|\mathscr{B}(\vec{v})-\mathscr{B}(\overrightarrow{\tilde{v}})\|_{L\left(X_{1}, \mathscr{H}\right)} \leq C_{B}\|\vec{v}-\overrightarrow{\tilde{v}}\|_{X_{0}} \quad \text { for all } \vec{v},, \overrightarrow{\tilde{v}} \in X_{0}
\end{aligned}
$$

(again to be verified for the nonlinear acoustics models from Section 2 in Section 5.3 below) and the fact that $\hat{\mathbf{u}}:=\mathbf{u}-\tilde{\mathbf{u}}:=\mathscr{T}(\mathbf{v})-\mathscr{T}(\tilde{\mathbf{v}})$ with $\mathbf{v}, \tilde{\mathbf{v}} \in B_{R}^{X}(0)$ (thus, by the already shown self-mapping property of $\mathscr{T}$, also $\mathbf{u}, \tilde{\mathbf{u}} \in B_{R}^{X}(0)$ ) satisfies (5.6) with b, $\mathrm{c}_{i}, \mathscr{G}$ as in (5.23),

$$
f=(\mathscr{B}(\mathbf{v})-\mathscr{B}(\tilde{\mathbf{v}}))[\tilde{\mathbf{u}}]+(\mathbf{A}(\mathbf{v})-\mathbf{A}(\tilde{\mathbf{v}}))_{m+1} \tilde{\mathbf{u}}
$$


and homogeneous initial data. Proposition 5.1 and the estimate

$$
\begin{aligned}
\|f\|_{L^{2}(0, T ; \mathscr{H}) \leq} & \|(\mathscr{B}(\mathbf{v})-\mathscr{B}(\tilde{\mathbf{v}}))[\tilde{\mathbf{u}}]\|_{L^{2}(0, T ; \mathscr{H})} \\
& +\| \sum_{i=1}^{r}\left(\mathrm{c}_{i}(\mathbf{v})-\mathrm{c}_{i}(\tilde{\mathbf{v}}) \mathscr{A}^{\ell_{i}} \tilde{u}_{m-1}\left\|_{L^{2}(0, T ; \mathscr{H})}+\right\|\left(\mathrm{b}(\mathbf{v})-\mathrm{b}(\tilde{\mathbf{v}}) \mathscr{A}^{k_{m}} \tilde{u}_{m} \|_{L^{2}(0, T ; \mathscr{H})}\right.\right. \\
\leq & \left(\int_{0}^{T}\|\mathscr{B}(\mathbf{v}(t))-\mathscr{B}(\tilde{\mathbf{v}})\|_{L\left(X_{1}, \mathscr{H}\right)}^{2}\|\tilde{\mathbf{u}}(t)\|_{X_{1}}^{2} d t\right)^{1 / 2} \\
& +\left(\int_{0}^{T} \| \sum_{i=1}^{r}\left(\mathrm{c}_{i}(\mathbf{v}(t))-\mathrm{c}_{i}(\tilde{\mathbf{v}}(t)) C_{P F}^{r-i} \|_{L(\mathscr{H}, \mathscr{H})}^{2}\left|\mathscr{A}^{\ell_{r}} \tilde{u}_{m-1}(t)\right|^{2} d t\right)^{1 / 2}\right. \\
& +\left(\int_{0}^{T}\|\mathrm{~b}(\mathbf{v}(t))-\mathrm{b}(\tilde{\mathbf{v}}(t))\|_{L(\mathscr{H}, \mathscr{H})}^{2}\left|\mathscr{A}^{k_{m}} \tilde{u}_{m}(t)\right|^{2} d t\right)^{1 / 2} \\
\leq & \left(C_{\mathrm{c}}+C_{\mathrm{b}}+C_{B}\right) R\|\mathbf{v}-\tilde{\mathbf{v}}\|_{X}
\end{aligned}
$$

yields contractivity for $R$ small enough. Thus from Banach's Fixed Point Theorem we obtain the following result.

Theorem 5.1. Under conditions (5.2), (5.24), (5.25), there exists $R>0$ (sufficiently small) such that for any initial data (5.22) with $\left\|\vec{u}_{0}\right\|_{X_{0}} \leq \frac{R}{C}$ the initial value problem (5.1), (5.22) has a unique solution $\mathbf{u}=\left(u, \partial_{t} u, \ldots, \partial_{t}^{m} u\right) \in X$ and this solution satisfies the estimate

$$
\left\|\left(u, \partial_{t} u, \ldots, \partial_{t}^{m} u\right)\right\|_{X} \leq C\left\|\vec{u}^{0}\right\|_{X_{0}}
$$

with C as in Proposition 5.1.

5.3. Application to models from nonlinear acoustics. We now verify conditions (5.24), (5.25), i.e.,

$$
\begin{aligned}
& \|\mathbf{v}\|_{X} \leq R \Rightarrow \mathrm{b}(\mathbf{v}), \mathrm{c}_{i}(\mathbf{v}) \in L^{\infty}(0, T ; L(\mathscr{H}, \mathscr{H})) \cap W^{1,1}(0, T ; L(\mathscr{H}, \mathscr{H})), \\
& \|\vec{v}\|_{X_{0}} \leq R \Rightarrow \\
& \left(\mathrm{b}(\vec{v}) \geq \underline{\mathrm{b}}>0 \quad \mathrm{c}_{i}(\vec{v}) \geq \underline{\mathrm{c}}_{i}>0\right. \\
& \left|\mathscr{A}^{\left(\ell_{i}-k_{m}\right) / 2}\left[\mathrm{c}_{i}(\vec{v}) \mathscr{A}^{\left(k_{m}+\ell_{i}\right) / 2} w_{m-1}\right]-\left[\mathrm{c}_{i}(\vec{v}) \mathscr{A}^{\ell_{i}} w_{m-1}\right]\right| \\
& +\left|\mathscr{A}^{\left(\ell_{r}-k_{m}\right) / 2}\left[\mathrm{~b}(\vec{v}) \mathscr{A}^{\left(k_{m}+\ell_{r}\right) / 2} w_{m-1}\right]-\left[\mathrm{b}(\vec{v}) \mathscr{A}^{\ell_{r}} w_{m-1}\right]\right| \\
& +\left|\mathscr{A}^{\left(\ell_{i}-\ell_{r}\right) / 2}\left[\mathrm{c}_{i}(\vec{v}) \mathscr{A}^{\left(\ell_{r}+\ell_{i}\right) / 2} w_{m-1}\right]-\left[\mathrm{c}_{i}(\vec{v}) \mathscr{A}^{\ell_{i}} w_{m-1}\right]\right| \\
& \leq C_{\mathrm{bc}}\left(\left|\mathscr{A}^{k_{m}} w_{m-1}\right|+\left|\mathscr{A}^{\left(k_{m}+\ell_{r}\right) / 2} w_{m-1}\right|\right)+c_{\mathrm{bc}}\left|\mathscr{A}^{\ell_{r}} w_{m-1}\right| \text {, for all } \vec{w} \in X_{1} \\
& \left.|\mathscr{B}(\vec{v})[\vec{w}]| \leq C_{G}\|\vec{w}\|_{X_{0}} \text { for all } \vec{w} \in X_{0}\right)
\end{aligned}
$$

and

$$
\begin{aligned}
& \sum_{i=1}^{r}\left\|\mathrm{c}_{i}(\vec{v})-\mathrm{c}_{i}(\overrightarrow{\tilde{v}})\right\|_{L(\mathscr{H}, \mathscr{H})} C_{P F}^{r-i} \leq C_{\mathrm{c}}\|\vec{v}-\overrightarrow{\tilde{v}}\|_{X_{0}}, \\
& \|\mathrm{~b}(\vec{v})-\mathrm{b}(\overrightarrow{\tilde{v}})\|_{L(\mathscr{H}, \mathscr{H})} \leq C_{\mathrm{b}}\|\vec{v}-\overrightarrow{\tilde{v}}\|_{X_{0}}, \\
& \|\mathscr{B}(\vec{v})-\mathscr{B}(\overrightarrow{\tilde{v}})\|_{L\left(X_{1}, \mathscr{H}\right)} \leq C_{B}\|\vec{v}-\overrightarrow{\tilde{v}}\|_{X_{0}} \text { for all } \vec{v}, \overrightarrow{\tilde{v}} \in X_{0}
\end{aligned}
$$

for the models from Section 2. 
BCBJK: $m=2, k_{m}=1, r=2, \ell_{1}=1, \ell_{r}=2$,

$$
\begin{aligned}
& X_{0}=\mathscr{D}\left(\mathscr{A}^{2}\right) \times \mathscr{D}\left(\mathscr{A}^{3 / 2}\right) \times \mathscr{D}\left(\mathscr{A}^{1 / 2}\right), \quad X_{1}=\mathscr{D}\left(\mathscr{A}^{2}\right) \times \mathscr{D}\left(\mathscr{A}^{2}\right) \times \mathscr{D}(\mathscr{A}), \\
& \mathrm{b}(\vec{v})=\frac{\beta_{1}}{\alpha\left(v_{1}\right)}, \quad \mathrm{c}_{1}(\vec{v})=\frac{\beta_{3}}{\alpha\left(v_{1}\right)}, \quad \mathrm{c}_{2}(\vec{v})=\frac{\beta_{2}}{\alpha\left(v_{1}\right)}, \quad \alpha\left(v_{1}\right)=1+\beta_{5} v_{1}, \\
& \mathscr{B}(\vec{v})[\vec{w}]=\frac{1}{\alpha\left(v_{1}\right)}\left(-\beta_{4} \mathscr{A}^{2} w_{0}+\beta_{5} v_{2} w_{2}+2 \nabla v_{1} \cdot \nabla w_{1}+2 \nabla v_{0} \cdot \nabla w_{2}\right) .
\end{aligned}
$$

Conditions (5.26), (5.27) follow from

$$
\left|\frac{d}{d t} \mathrm{~b}(\mathbf{v})(t)\right|_{L(\mathscr{H}, \mathscr{H})}=\beta_{1} \beta_{5}\left|\frac{1}{\alpha\left(v_{1}(t)\right)^{2}} v_{2}(t)\right|_{L^{\infty}(\Omega)} \leq \beta_{1} \beta_{5}\left|\frac{1}{\alpha\left(v_{1}(t)\right)}\right|_{L^{\infty}(\Omega)}^{2}\left|v_{2}(t)\right|_{L^{\infty}(\Omega)}
$$

and likewise for $\mathrm{c}_{1}, \mathrm{c}_{2}$, as well as the estimates

$$
\begin{aligned}
&\left|v_{2}\right|_{L^{1}\left(0, T ; L^{\infty}(\Omega)\right)} \leq\left.\sqrt{T} C_{\mathscr{D}(\mathscr{A}) \rightarrow L^{\infty}(\Omega)}|| \mathscr{A} v_{2}\right|_{L^{2}(0, T ; \mathscr{H})} \leq \sqrt{T} C_{\mathscr{D}(\mathscr{A}) \rightarrow L^{\infty}(\Omega)} R \\
&\left|\frac{1}{\alpha\left(v_{1}\right)}\right|_{L^{\infty}\left(0, T ; L^{\infty}(\Omega)\right.} \leq \frac{1}{1-\left|1-\alpha\left(v_{1}\right)\right|_{L^{\infty}\left(0, T ; L^{\infty}(\Omega)\right.}} \\
&\left|1-\alpha\left(v_{1}\right)\right|_{L^{\infty}\left(0, T ; L^{\infty}(\Omega)\right.} \leq\left.\beta_{5} C_{\mathscr{D}\left(\mathscr{A}^{3 / 2}\right) \rightarrow L^{\infty}(\Omega)}|| \mathscr{A}_{1}\right|_{L^{\infty}(0, T ; \mathscr{H})} \\
& \leq \beta_{5} C_{\mathscr{D}\left(\mathscr{A}^{3 / 2}\right) \rightarrow L^{\infty}(\Omega)} R \\
& \mid \mathscr{A}^{1 / 2}\left[\frac{1}{\alpha\left(v_{1}\right)} \mathscr{A}^{3 / 2} w_{m-1}\right]-\left[\frac{1}{\alpha\left(v_{1}\right)} \mathscr{A}^{2} w_{m-1}\right] \mid \\
&=\left|\nabla\left[\frac{1}{\alpha\left(v_{1}\right)} \nabla \Delta w_{m-1}\right]-\left[\frac{1}{\alpha\left(v_{1}\right)} \Delta^{2} w_{m-1}\right]\right|=\frac{1}{c^{2}} \frac{B}{A}\left|\frac{1}{\alpha\left(v_{1}\right)^{2}} \nabla v_{1} \cdot \nabla \Delta w_{m-1}\right| \\
& \leq \frac{1}{c^{2}} \frac{B}{A}\left|\frac{1}{\alpha\left(v_{1}\right)^{2}}\right|_{L(\mathscr{H}, \mathscr{H})} C_{\mathscr{D}(\mathscr{A}) \rightarrow L^{\infty}(\Omega)}\left|\mathscr{A}^{3 / 2} v_{1}\right|\left|\mathscr{A}^{3 / 2} w_{m-1}\right| \\
& \leq \frac{1}{c^{2}} \frac{B}{A}\left|\frac{1}{\alpha\left(v_{1}\right)^{2}}\right|_{L(\mathscr{H}, \mathscr{H})} C_{\mathscr{D}(\mathscr{A}) \rightarrow L^{\infty}(\Omega)} R\left|\mathscr{A}^{\left(k_{m}+\ell_{r}\right) / 2} w_{m-1}\right| \\
&\left|\mathscr{A}^{-1 / 2}\left[\frac{1}{\alpha\left(v_{1}\right)} \mathscr{A}^{3 / 2} w_{m-1}\right]-\left[\frac{1}{\alpha\left(v_{1}\right)} \mathscr{A} w_{m-1}\right]\right| \\
&=\left|\mathscr{A}^{-1 / 2}\left(\left[\frac{1}{\alpha\left(v_{1}\right)} \nabla \Delta w_{m-1}\right]-\nabla\left[\frac{1}{\alpha\left(v_{1}\right)} \Delta w_{m-1}\right]\right)\right| \\
&=\frac{1}{c^{2}} \frac{B}{A}\left|\mathscr{A}^{-1 / 2}\left[\frac{1}{\alpha\left(v_{1}\right)^{2}} \nabla v_{1} \Delta w_{m-1}\right]\right| \\
& \leq C_{P F} \frac{1}{c^{2}} \frac{B}{A}\left|\frac{1}{\alpha\left(v_{1}\right)^{2}}\right|_{L(\mathscr{H}, \mathscr{H})} C_{\mathscr{D}(\mathscr{A}) \rightarrow L^{\infty}(\Omega)} R\left|\mathscr{A}^{\left(k_{m}+\ell_{r}\right) / 2} w_{m-1}\right|
\end{aligned}
$$

$|\mathscr{B}(\vec{v})[\vec{w}]|$

$$
\begin{aligned}
& \leq\left|\frac{1}{\alpha\left(v_{1}\right)}\right|_{L(\mathscr{H}, \mathscr{H})}\left(\beta_{4}\left|\mathscr{A}^{2} w_{0}\right|+\beta_{5}\left|v_{2} w_{2}\right|+2\left|\nabla v_{1} \cdot \nabla w_{1}\right|+2\left|\nabla v_{0} \cdot \nabla w_{2}\right|\right) \\
& \leq\left|\frac{1}{\alpha\left(v_{1}\right)}\right|_{L(\mathscr{H}, \mathscr{H})}\left(\beta_{4}\left|\mathscr{A}^{2} w_{0}\right|+\beta_{5} C_{\mathscr{D}\left(\mathscr{A}^{1 / 2}\right) \rightarrow L^{4}(\Omega)}^{2}\left|\mathscr{A}^{1 / 2} v_{2}\right|\left|\mathscr{A}^{1 / 2} w_{2}\right|\right. \\
& \left.\quad+2 C_{\mathscr{D}(\mathscr{A}) \rightarrow W^{1,4}(\Omega)}^{2}\left|\mathscr{A} v_{1}\right|\left|\mathscr{A} w_{1}\right|+2 C_{\mathscr{D}(\mathscr{A}) \rightarrow L^{\infty}(\Omega)}\left|\mathscr{A}^{3 / 2} v_{0}\right|\left|\mathscr{A}^{1 / 2} w_{2}\right|\right) \\
& \leq\left|\frac{1}{\alpha\left(v_{1}\right)}\right|_{L(\mathscr{H}, \mathscr{H})}\|\vec{w}\|_{X_{0}} \\
& \quad\left(\beta_{4}+R\left(\beta_{5} C_{\mathscr{D}\left(\mathscr{A}^{1 / 2}\right) \rightarrow L^{4}(\Omega)}^{2}+2 C_{\mathscr{D}(\mathscr{A}) \rightarrow W^{1,4}(\Omega)}^{2} C_{P F}+2 C_{\mathscr{D}(\mathscr{A}) \rightarrow L^{\infty}(\Omega)} \mid\right)\right) .
\end{aligned}
$$


Condition (5.28) follows from the estimates

$$
\begin{aligned}
& \left|\frac{1}{\alpha\left(v_{1}\right)}-\frac{1}{\alpha\left(\tilde{v}_{1}\right)}\right|_{L(\mathscr{H}, \mathscr{H})}=\beta_{5}\left|\frac{1}{\alpha\left(v_{1}\right) \alpha\left(\tilde{v}_{1}\right)}\left(v_{1}-\tilde{v}_{1}\right)\right|_{L^{\infty}(\Omega)} \\
& \leq \beta_{5}\left|\frac{1}{\alpha\left(v_{1}\right)}\right|_{L^{\infty}(\Omega)}\left|\frac{1}{\alpha\left(\tilde{v}_{1}\right)}\right|_{L^{\infty}(\Omega)} C_{\mathscr{D}\left(\mathscr{A}^{3} / 2\right) \rightarrow L^{\infty}(\Omega)}\|\vec{v}-\overrightarrow{\tilde{v}}\|_{X_{0}}
\end{aligned}
$$

as well as the identity

$$
\begin{aligned}
\mathscr{B}(\vec{v}) \vec{w}-\mathscr{B}(\overrightarrow{\tilde{v}}) \vec{w}=\frac{1}{\alpha\left(\tilde{v}_{1}\right)} & \left(\left(\alpha\left(\tilde{v}_{1}\right)-\alpha\left(v_{1}\right)\right) \mathscr{B}(\vec{v}) \vec{w}\right. \\
& \left.\quad \beta_{5}\left(v_{2}-\tilde{v}_{2}\right) w_{2}+2 \nabla\left(v_{1}-\tilde{v}_{1}\right) \cdot \nabla w_{1}+2 \nabla\left(v_{0}-\tilde{v}_{0}\right) \cdot \nabla w_{2}\right)
\end{aligned}
$$

and the estimates (5.29), (5.30), (5.31), as well as (analogously to (5.30))

$$
\begin{gathered}
\left|\left(v_{2}-\tilde{v}_{2}\right) w_{2}\right| \leq C_{\mathscr{D}\left(\mathscr{A}^{1 / 2}\right) \rightarrow L^{4}(\Omega)}^{2}\left|\mathscr{A}^{1 / 2}\left(v_{2}-\tilde{v}_{2}\right)\right|\left|\mathscr{A}^{1 / 2} w_{2}\right|, \\
\left|\nabla\left(v_{1}-\tilde{v}_{1}\right) \cdot \nabla w_{1}\right| \leq C_{\mathscr{D}(\mathscr{A}) \rightarrow W^{1,4}(\Omega)}^{2}\left|\mathscr{A}\left(v_{1}-\tilde{v}_{1}\right)\right|\left|\mathscr{A} w_{1}\right|,
\end{gathered}
$$

and

$$
\left|\nabla\left(v_{0}-\tilde{v}_{0}\right) \cdot \nabla w_{2}\right| \leq C_{\mathscr{D}(\mathscr{A}) \rightarrow L^{\infty}(\Omega)}\left|\mathscr{A}^{3 / 2}\left(v_{0}-\tilde{v}_{0}\right)\right|\left|\mathscr{A}^{1 / 2} w_{2}\right|,
$$

hence altogether

$$
|\mathscr{B}(\vec{v}) \vec{w}-\mathscr{B}(\overrightarrow{\tilde{v}}) \vec{w}| \leq C_{B}\|\vec{v}-\overrightarrow{\tilde{v}}\|_{X_{0}}\|\vec{w}\|_{X_{0}}
$$

JMGT:. $m=2, k_{m}=0, r=1, \ell_{r}=1$

$$
\begin{aligned}
& X_{0}=\mathscr{D}(\mathscr{A}) \times \mathscr{D}\left(\mathscr{A}^{1 / 2}\right) \times \mathscr{H}, \quad X_{1}=\mathscr{D}(\mathscr{A}) \times \mathscr{D}(\mathscr{A}) \times \mathscr{H}, \\
& \mathrm{b}(\vec{v})=\frac{\alpha\left(v_{0}\right)}{T_{\text {rel }}}, \quad \mathrm{c}(\vec{v})=\frac{b}{T_{\text {rel }}}, \quad \alpha\left(v_{0}\right)=1-\frac{2 \beta_{a}}{\rho c^{2}} v_{0}, \\
& \mathscr{B}(\vec{v})[\vec{w}]=-\frac{c^{2}}{T_{r e l}} \mathscr{A} w_{0}+\frac{2 \beta_{a}}{\rho c^{2}} v_{1} w_{1} .
\end{aligned}
$$


Conditions (5.26) and (5.27) follow from

$$
\begin{aligned}
& \left.\left\|\frac{1}{T_{\text {rel }}}-\mathrm{b}(\mathbf{v})(t)\right\|_{L^{\infty}(0, T ; L(\mathscr{H}, \mathscr{H}))}\right)=\frac{1}{T_{\text {rel }}}\left\|1-\alpha\left(v_{0}\right)\right\|_{L^{\infty}\left(0, T ; L^{\infty}(\Omega)\right)} \\
& \leq \frac{1}{T_{\text {rel }}} \frac{2 \beta_{a}}{\rho c^{2}} \|\left. v_{0}(t)\right|_{L^{\infty}\left(0, T ; L^{\infty}(\Omega)\right.} \leq \frac{1}{T_{\text {rel }}} \frac{2 \beta_{a}}{\rho c^{2}} C_{\mathscr{D}(\mathscr{A}) \rightarrow L^{\infty}(\Omega)} R \\
& \left\|\frac{d}{d t} \mathrm{~b}(\mathbf{v})(t)\right\|_{L^{1}(0, T ; L(\mathscr{H}, \mathscr{H}))}=\frac{1}{T_{\text {rel }}} \frac{2 \beta_{a}}{\rho c^{2}}\left\|v_{1}\right\|_{L^{1}\left(0, T ; L^{\infty}(\Omega)\right)} \leq \frac{1}{T_{r e l}} \frac{2 \beta_{a}}{\rho c^{2}} \sqrt{T} C_{\mathscr{D}(\mathscr{A}) \rightarrow L^{\infty}(\Omega)} R \\
& \mid \mathscr{A}^{1 / 2}\left[\alpha\left(v_{0}\right) \mathscr{A}^{1 / 2} w_{m-1}-\left[\alpha\left(v_{0}\right) \mathscr{A} w_{m-1} \mid\right.\right. \\
& =\mid \nabla\left[\alpha\left(v_{0}\right) \nabla w_{1}-\left[\alpha\left(v_{0}\right) \Delta w_{m-1}\left|=\frac{2 \beta_{a}}{\rho c^{2}}\right| \nabla v_{0} \cdot \nabla w_{m-1} \mid\right.\right. \\
& \leq \frac{2 \beta_{a}}{\rho c^{2}} C_{\mathscr{D}(\mathscr{A}) \rightarrow W^{1,4}(\Omega)}^{2}\left|\mathscr{A} v_{0}\right|\left|\mathscr{A} w_{m-1}\right| \leq \frac{2 \beta_{a}}{\rho c^{2}} C_{\mathscr{D}(\mathscr{A}) \rightarrow W^{1,4}(\Omega)}^{2} R\left|\mathscr{A}^{\ell_{r}} w_{m-1}\right| \\
& |\mathscr{B}(\vec{v})[\vec{w}]| \leq \frac{c^{2}}{T_{\text {rel }}}\left|\mathscr{A} w_{0}\right|+\frac{2 \beta_{a}}{\rho c^{2}}\left|v_{1} w_{1}\right| \\
& \leq \frac{c^{2}}{T_{\text {rel }}}\left|\mathscr{A} w_{0}\right|+\frac{2 \beta_{a}}{\rho c^{2}} C_{\mathscr{D}(\mathscr{A} 1 / 2) \rightarrow L^{4}(\Omega)}^{2}\left|\mathscr{A}^{1 / 2} v_{1}\right|\left|\mathscr{A}^{1 / 2} w_{1}\right| \\
& \leq\left(\frac{c^{2}}{T_{\text {rel }}}+\frac{2 \beta_{a}}{\rho c^{2}} C_{\mathscr{D}\left(\mathscr{A}^{1 / 2}\right) \rightarrow L^{4}(\Omega)}^{2} R\right)\|\vec{w}\|_{X_{0}},
\end{aligned}
$$

and condition (5.28) from

$$
\begin{aligned}
& \left|\alpha\left(v_{0}\right)-\alpha\left(\tilde{v}_{0}\right)\right|_{L(\mathscr{H}, \mathscr{H})}=\frac{2 \beta_{a}}{\rho c^{2}}\left|v_{0}-\tilde{v}_{0}\right|_{L^{\infty}(\Omega)} \leq \frac{2 \beta_{a}}{\rho c^{2}} C_{\mathscr{D}(\mathscr{A}) \rightarrow L^{\infty}(\Omega)}^{2}\left|\mathscr{A}\left(v_{0}-\tilde{v}_{0}\right)\right| \\
& \leq \frac{2 \beta_{a}}{\rho c^{2}} C_{\mathscr{D}(\mathscr{A}) \rightarrow L^{\infty}(\Omega)}^{2}\|\vec{v}-\overrightarrow{\tilde{v}}\|_{X_{0}} \\
& |\mathscr{B}(\vec{v})[\vec{w}]-\mathscr{B}(\overrightarrow{\tilde{v}})[\vec{w}]|=\frac{2 \beta_{a}}{\rho c^{2}}\left|\left(v_{1}-\tilde{v}_{1}\right) w_{1}\right| \leq C_{\mathscr{D}\left(\mathscr{A}^{1 / 2}\right) \rightarrow L^{4}(\Omega)}^{2}\left|\mathscr{A}^{1 / 2}\left(v_{1}-\tilde{v}_{1}\right)\right|\left|\mathscr{A}^{1 / 2} w_{1}\right| \\
& \leq C_{\mathscr{D}\left(\mathscr{A}^{1 / 2}\right) \rightarrow L^{4}(\Omega)}^{2}\|\vec{v}-\overrightarrow{\tilde{v}}\|_{X_{0}}\|\vec{w}\|_{X_{0}} .
\end{aligned}
$$

Westervelt:. $m=1, k_{m}=1, r=1, \ell_{r}=1$

$$
\begin{aligned}
& X_{0}=\mathscr{D}(\mathscr{A}) \times \mathscr{D}\left(\mathscr{A}^{1 / 2}\right), \quad X_{1}=\mathscr{D}(\mathscr{A}) \times \mathscr{D}(\mathscr{A}), \\
& \mathrm{b}(\vec{v})=\frac{b}{\alpha\left(v_{0}\right)}, \quad \mathrm{c}(\vec{v})=\frac{c^{2}}{\alpha\left(v_{0}\right)}, \quad \alpha\left(v_{0}\right)=1-\frac{2 \beta_{a}}{\rho c^{2}} v_{0}, \\
& \mathscr{B}(\vec{v})[\vec{w}]=\frac{2 \beta_{a}}{\rho c^{2}} v_{1} w_{1} .
\end{aligned}
$$

Conditions (5.26), (5.27), (5.28) can be verified as for JMGT, just skipping the term $-\frac{c^{2}}{T_{\text {rel }}} \mathscr{A} w_{0}$ in $B$ and taking into account the fact that $\frac{1}{\alpha\left(v_{0}\right)}-\frac{1}{\alpha\left(\tilde{v}_{0}\right)}=-\frac{1}{\alpha\left(v_{0}\right) \alpha\left(\tilde{v}_{0}\right)}\left(\alpha\left(v_{0}\right)-\alpha\left(\tilde{v}_{0}\right)\right)$.

Corollary 5.1. There exists $R>0$ (sufficiently small) such that for any initial data (5.22) with $\left\|\vec{u}_{0}\right\|_{X_{0}} \leq \frac{R}{C}$ the initial value problems (2.3), (2.2), (with $m=2$ ) (2.1) (with $m=1$ ), (5.22) have unique solutions $u \in \tilde{X}$ and these solutions satisfy the estimate

$$
\|u\|_{\tilde{X}} \leq C\left\|\vec{u}^{0}\right\|_{X_{0}}
$$


with

BCBJK: $\tilde{X}=W^{2, \infty}\left(0, T ; H_{0}^{1}(\Omega)\right) \cap H^{2}\left(0, T ; H_{\diamond}^{2}(\Omega)\right)$

$$
\begin{aligned}
& \cap W^{1, \infty}\left(0, T ; H_{\diamond}^{3}(\Omega)\right) \cap H^{1}\left(0, T ; H_{\diamond}^{4}(\Omega)\right), \\
& X_{0}=H_{\diamond}^{4}(\Omega) \times H_{\diamond}^{3}(\Omega) \times H_{0}^{1}(\Omega)
\end{aligned}
$$

JMGT: $\tilde{X}=W^{2, \infty}\left(0, T ; L^{2}(\Omega)\right) \cap W^{1, \infty}\left(0, T ; H_{0}^{1}(\Omega)\right) \cap H^{1}\left(0, T ; H_{\diamond}^{2}(\Omega)\right)$,

$$
X_{0}=H_{\diamond}^{2}(\Omega) \times H_{0}^{1}(\Omega) \times L^{2}(\Omega)
$$

Westervelt: $\tilde{X}=W^{1, \infty}\left(0, T ; H_{0}^{1}(\Omega)\right) \cap H^{1}\left(0, T ; H_{\diamond}^{2}(\Omega)\right)$,

$$
X_{0}=H_{\diamond}^{2}(\Omega) \times H_{0}^{1}(\Omega)
$$

where $H_{\diamond}^{2}(\Omega)=H^{2}(\Omega) \cap H_{0}^{1}(\Omega), H_{\diamond}^{3}(\Omega)=H^{3}(\Omega) \cap H_{0}^{1}(\Omega), H_{\diamond}^{4}(\Omega)=\left\{v \in H^{4}(\Omega): v, \Delta v \in\right.$ $\left.H_{0}^{1}(\Omega)\right\}$.

Remark 5.2. A comparison to $[17,18,21]$ where the following regularity results have been established

$$
\begin{aligned}
& u \in W^{2, \infty}\left(0, T ; H_{0}^{1}(\Omega)\right) \cap H^{2}\left(0, T ; H_{\diamond}^{2}(\Omega)\right) \cap L^{\infty}\left(0, T ; H_{\diamond}^{3}(\Omega)\right), \\
& \left(u_{0}, u_{1}, u_{2}\right) \in H_{\diamond}^{3}(\Omega) \times H_{\diamond}^{3}(\Omega) \times H_{0}^{1}(\Omega) \quad \text { for BCBJK; } \\
& u \in W^{2, \infty}\left(0, T ; L^{2}(\Omega)\right) \cap W^{1, \infty}\left(0, T ; H_{0}^{1}(\Omega)\right) \cap L^{\infty}\left(0, T ; H_{\diamond}^{2}(\Omega)\right), \\
& \left(u_{0}, u_{1}, u_{2}\right) \in H_{\diamond}^{2}(\Omega) \times H_{0}^{1}(\Omega) \times L^{2}(\Omega) \quad \text { for JMGT; } \\
& u \in H^{2}\left(0, T ; H_{0}^{1}(\Omega)\right) \cap W^{2, \infty}\left(0, T ; L^{2}(\Omega)\right) \cap W^{1, \infty}\left(0, T ; H_{0}^{1}(\Omega)\right) \cap L^{\infty}\left(0, T ; H_{\diamond}^{2}(\Omega)\right), \\
& \left(u_{0}, u_{1}\right) \in H_{\diamond}^{2}(\Omega) \times H_{\diamond}^{2}(\Omega) \quad \text { for Westervelt; }
\end{aligned}
$$

shows that our results here give stronger regularity under stronger (or the same) smoothness of the initial data for BCBJK and JMGT, and weaker regularity under weaker smoothness of the initial data for Westervelt. Beyond such a comparison, our aim is a unified approach that is amenable to implicit time stepping schemes, though, as we will carry out analogous energy estimates in the following section.

\section{Energy Estimates, Well-Posedness And Convergence of Time-Discretised}

\section{SYSTEMS}

In this section, we will transfer the energy estimates from Section 5 to the systems obtained by an implicit discretisation with stiffly accurate Runge Kutta methods. This will enable us to prove well- posedness of the (time) discretised problems. As the most transparent special case, we will first of all study the implicit Euler scheme.

6.1. Euler scheme. For a fixed time grid $t_{0}<t_{1}<\cdots<t_{N}$ which for simplicity of exposition we choose equidistant $t_{i}=i \tau, \tau=\frac{T}{N}$ we replace a time dependent function $u:[0, T] \rightarrow \mathbb{R}$ by a vector $\underline{u}_{\tau}=\left(u^{(0)}, \ldots, u^{(N)}\right)$ of approximations at the time instances. With some time discretisation $D_{t}^{(n)} \underline{u}_{\tau} \approx u^{\prime}\left(t_{n}\right)$, e.g., according to a backward Euler scheme $D_{t}^{(n+1)}:=\mathrm{d}_{t}^{(n+1)}$ where

$$
\mathrm{d}_{t}^{(n+1)} \underline{u}_{\tau}:=\frac{1}{\tau}\left(u^{(n+1)}-u^{(n)}\right)
$$


we apply this component wise to the grid version $\underline{\mathbf{u}}_{\tau}=\left(\mathbf{u}^{0}, \ldots, \mathbf{u}^{N}\right)$ of $\mathbf{u}$ to obtain an implicit time discretisation of (5.3)

$$
D_{t}^{(n+1)} \underline{\mathbf{u}}_{\tau}+\mathbf{A}\left(\mathbf{u}^{(n+1)}\right) \mathbf{u}^{(n+1)}=\left(0, \cdots, 0, \mathscr{B}\left(\mathbf{u}^{(n+1)}\right)\left[\mathbf{u}^{(n+1)}\right]\right)^{T},
$$

and of (5.6)

$$
\left.D_{t}^{(n+1)} \underline{\mathbf{u}}_{\tau}+\mathbf{A}^{(n+1)} \mathbf{u}^{(n+1)}=\left(0, \cdots, 0, \mathscr{G}^{(n+1)}\left[\mathbf{u}^{(n+1)}\right]+f^{(n+1)}\right)\right)^{T},
$$

respectively.

Time discrete counterparts of the function spaces in Section 5 can be defined by setting, for some Hilbert space $Z$ (of space dependent functions)

$$
\begin{gathered}
\left\|\underline{u}_{\tau}\right\|_{L_{\tau}^{p}(Z)}:=\left(\tau \sum_{n=0}^{N}\left\|u^{(n)}\right\|_{Z}^{p}\right)^{1 / p} \text { for } 1 \leq p<\infty, \quad\left\|\underline{u}_{\tau}\right\|_{L_{\tau}^{\infty}(Z)}:=\max _{n \in\{0, \ldots, N\}}\left\|u^{(n)}\right\|_{Z}, \\
\left\|\underline{u}_{\tau}\right\|_{W_{\tau}^{1, p}(Z)}:=\left\|\underline{\mathrm{d}}_{t} \underline{u}_{\tau}\right\|_{L_{\tau}^{p}(Z)}
\end{gathered}
$$

that satisfy the $\tau$ independent estimates (using the crude estimate $(N+1) \tau \leq 2 T$ )

$$
\left\|\underline{u}_{\tau}\right\|_{L_{\tau}^{p}(Z)} \leq(2 T)^{1 / p}\left\|\underline{u}_{\tau}\right\|_{L_{\tau}^{\infty}(Z)}, \quad\left\|\underline{u}_{\tau}\right\|_{L_{\tau}^{\infty}(Z)} \leq\left\|u^{0}\right\|_{Z}+T^{(p-1) / p}\left\|\underline{u}_{\tau}\right\|_{W_{\tau}^{1, p}(Z)},
$$

where the latter follows from Hölder's inequality and the inverse triangle inequality

$$
\begin{aligned}
\left\|\underline{\mathrm{d}}_{t} \underline{u}_{\tau}\right\|_{L_{\tau}^{p}(Z)} & =\left(\tau^{1-p} \sum_{n=0}^{N-1}\left\|u^{(n+1)}-u^{(n)}\right\|_{Z}^{p}\right)^{1 / p} \geq \tau^{1 / p-1} N^{1 / p-1} \sum_{n=0}^{N-1}\left\|u^{(n+1)}-u^{(n)}\right\|_{Z} \\
& \geq T^{1 / p-1} \sum_{n=0}^{N-1}\left(\left\|u^{(n+1)}\right\|_{Z}-\left\|u^{(n)}\right\|_{Z}\right) \geq T^{1 / p-1}\left(\left\|u^{N}\right\|_{Z}-\left\|u^{0}\right\|_{Z}\right) .
\end{aligned}
$$

Therewith we define, analogously to (5.17) and using the identity $\frac{\mathrm{d}}{\mathrm{d} t}^{(n)} \underline{u}_{j}=u_{j+1}^{(n)}$

$$
\begin{aligned}
X_{\tau}= & \left(L_{\tau}^{\infty}\left(\mathscr{D}\left(\mathscr{A}^{\max \left\{k_{m}, \ell_{r}\right\}}\right) \cap H_{\tau}^{1}\left(\mathscr{D}\left(\mathscr{A}^{\max \left\{k_{m}, \ell_{r}\right\}}\right)\right)\right)^{m-1}\right. \\
& \times L_{\tau}^{2}\left(\mathscr{D}\left(\mathscr{A}^{\max \left\{k_{m}, \ell_{r}\right\}}\right)\right) \cap L_{\tau}^{\infty}\left(\mathscr{D}\left(\mathscr{A}^{\max \left\{k_{m},\left(k_{m}+\ell_{r}\right) / 2\right\}}\right)\right) \cap H_{\tau}^{1}\left(\mathscr{D}\left(\mathscr{A}^{k_{m}}\right)\right) \\
& \times L_{\tau}^{2}\left(\mathscr{D}\left(\mathscr{A}^{k_{m}}\right)\right) \cap L_{\tau}^{\infty}\left(\mathscr{D}\left(\mathscr{A}^{k_{m} / 2}\right)\right) \\
\subseteq & L_{\tau}^{\infty}\left(X_{0}\right) \cap L_{\tau}^{2}\left(X_{1}\right) .
\end{aligned}
$$

For obtaining energy estimates the inequality

$$
\left(D_{t}^{(n+1)} \underline{u}_{\tau} \mid u^{(n+1)}\right) \geq \frac{1}{2 \tau}\left(\left|u^{(n+1)}\right|^{2}-\left|u^{(n)}\right|^{2}\right)=\frac{1}{2} \mathrm{~d}_{t}^{(n+1)} \underline{|u|^{2}} \tau
$$

substituting its continuous counterpart $\left(u^{\prime}(t) \mid u(t)\right)=\frac{1}{2} \frac{d}{d t}|u|^{2}(t)$ will be crucial, which holds in the implicit Euler case $D_{t}^{(n+1)}=\mathrm{d}_{t}^{(n+1)}$ due to

$$
(v-w \mid v)=\frac{1}{2}\left(|v|^{2}-|w|^{2}+|v-w|^{2}\right) \geq \frac{1}{2}\left(|v|^{2}-|w|^{2}\right)
$$

[35, Eqns. (7.5.9), (7.5.10) p. 197]) but also for certain Runge Kutta methods, see [1, 2].

Energy estimates for (6.3) can be derived analogoulsy to those for (5.6) by testing with

$$
\left(0, \ldots, 0, \sum_{i=1}^{r} \mathscr{A}^{k_{m}}\left[\mathrm{c}_{i}^{(n+1)} \mathscr{A}^{\ell_{i}} u_{m-1}^{(n+1)}\right], \mathscr{A}^{k_{m}} u_{m}^{(n+1)}\right)^{T}
$$


and

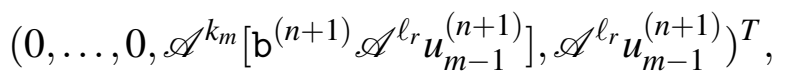

respectively, cf. (5.7), (5.14), applying (6.5), substituting (5.12) by

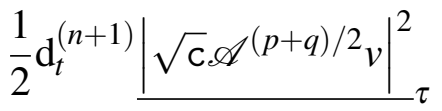

$$
\begin{aligned}
& =\frac{1}{2 \tau}\left(\left(\mathscr{A}^{(p+q) / 2} v^{(n+1)} \mid \mathrm{c}^{(n+1)} \mathscr{A}^{(p+q) / 2} v^{(n+1)}\right)-\left(\mathscr{A}^{(p+q) / 2} v^{(n)} \mid \mathrm{c}^{(n)} \mathscr{A}^{(p+q) / 2} v^{(n)}\right)\right) \\
& =\frac{1}{2}\left(\mathrm{~d}_{t}^{(n+1)} \underline{v}_{\tau} \mid \mathscr{A}^{(p+q) / 2}\left[\mathrm{c}^{(n+1)} \mathscr{A}^{(p+q) / 2}\left(v^{(n+1)}+v^{(n)}\right)\right]\right) \\
& +\frac{1}{2}\left(v^{(n+1)} \mid \mathscr{A}^{(p+q) / 2}\left[\left(\mathrm{~d}_{t}^{(n+1)} \underline{\mathrm{c}}_{\tau}\right) \mathscr{A}^{(p+q) / 2} v^{(n)}\right]\right) \\
& =\left(D_{t}^{(n+1)} \underline{v}_{\tau} \mid \mathscr{A}^{p}\left[\mathrm{c}^{(n+1)} \mathscr{A}^{q} v^{(n+1)}\right]\right) \\
& +\left(D_{t}^{(n+1)} \underline{v}_{\tau} \mid \mathscr{A}^{(p+q) / 2}\left[\mathrm{c}^{(n+1)} \mathscr{A}^{(p+q) / 2} v^{(n+1)}\right]-\mathscr{A}^{p}\left[\mathrm{c}^{(n+1)} \mathscr{A}^{q} v^{(n+1)}\right]\right) \\
& +\left(\mathrm{d}_{t}^{(n+1)} \underline{v}_{\tau}-D_{t}^{(n+1)} \underline{v}_{\tau} \mid \mathscr{A}^{(p+q) / 2}\left[\mathrm{c}^{(n+1)} \mathscr{A}^{(p+q) / 2} v^{(n+1)}\right]\right) \\
& -\tau\left(\mathrm{d}_{t}^{(n+1)} \underline{v}_{\tau} \mid \mathscr{A}^{(p+q) / 2}\left[\mathrm{c}^{(n+1)} \mathscr{A}^{(p+q) / 2} \mathrm{~d}_{t}^{(n+1)} \underline{v}_{\tau}\right]\right) \\
& +\frac{1}{2}\left(v^{(n+1)} \mid \mathscr{A}^{(p+q) / 2}\left[\left(\mathrm{~d}_{t}^{(n+1)} \underline{\mathrm{c}}_{\tau}\right) \mathscr{A}^{(p+q) / 2} v^{(n)}\right)\right.
\end{aligned}
$$

and using $D_{t}^{(n+1)} \underline{u}_{\tau, m-1}=u_{m}^{(n+1)}$.

The term $\tau\left(\left.\mathrm{d}_{t}^{(n+1)} \underline{v}_{\tau}\left|\mathscr{A}^{(p+q) / 2}\left[\mathrm{c}^{(n+1)} \mathscr{A}^{(p+q) / 2} \mathrm{~d}_{t}^{(n+1)} \underline{v}_{\tau}\right)=\tau\right| \sqrt{\mathrm{c}^{(n+1)}} \mathscr{A}^{(p+q) / 2} \mathrm{~d}_{t}^{(n+1)} \underline{v}_{\tau}\right|^{2}\right.$ is nonnegative and can therefore be skipped in the estimate.

The additional term containing $\mathrm{d}_{t}^{(n+1)} \underline{v}_{\tau}-D_{t}^{(n+1)} \underline{v}_{\tau}$ in (6.6) clearly vanishes in case an implicit Euler discretisation is used; otherwise it can be individually estimated.

Altogether this yields, in place of (5.13), (5.15)

$$
\begin{aligned}
& \frac{1}{2} \mathrm{~d}_{t}^{(n+1)} \underline{\left|\mathscr{A}^{k_{m} / 2} u_{m}\right|^{2}} \tau+\frac{1}{2} \sum_{i=1}^{r} \mathrm{~d}_{t}^{(n+1)} \underline{\left|\sqrt{\mathrm{c}_{i}} \mathscr{A}^{\left(k_{m}+\ell_{i}\right) / 2} u_{m-1}\right|^{2}} \tau+\left|\sqrt{\mathrm{b}^{(n+1)}} \mathscr{A}^{k_{m}} u_{m}^{(n+1)}\right|^{2} \\
& \leq \\
& \quad \frac{1}{\underline{\mathrm{b}}}\left|\mathscr{G}^{(n+1)}\left[\mathbf{u}^{(n+1)}\right]+f^{(n+1)}\right|^{2}+\frac{1}{4}\left|\sqrt{\mathrm{b}^{(n+1)}} \mathscr{A}^{k_{m}} u_{m}^{(n+1)}\right|^{2} \\
& \quad+\frac{1}{2} \sum_{i=1}^{r} \frac{1}{\underline{\mathrm{c}}_{i}}\left|\left(\mathrm{~d}_{t}^{(n+1)} \underline{\mathrm{c}}_{i}\right)\right|_{L(\mathscr{H}, \mathscr{H})}\left|\sqrt{\mathrm{c}_{i}^{(n+1)}} \mathscr{A}^{\left(k_{m}+\ell_{i}\right) / 2} u_{m-1}^{(n+1)}\right|^{2} \\
& \quad+\frac{1}{4}\left|\sqrt{\mathrm{b}^{(n+1)}} \mathscr{A}^{k_{m}} u_{m}^{(n+1)}\right|^{2} \\
& \quad+\frac{1}{\underline{\mathrm{b}}} \sum_{i=1}^{r}\left|\mathscr{A}^{\left(\ell_{i}-k_{m}\right) / 2}\left[\mathrm{c}_{i}^{(n+1)} \mathscr{A}^{\left(k_{m}+\ell_{i}\right) / 2} u_{m-1}^{(n+1)}\right]-\left[\mathrm{c}_{i}^{(n+1)} \mathscr{A}^{\ell_{i}} u_{m-1}^{(n+1)}\right]\right|^{2}
\end{aligned}
$$




$$
\begin{aligned}
& \frac{1}{2} \mathrm{~d}_{t}^{(n+1)} \underline{\left|\sqrt{\mathrm{b}} \mathscr{A}^{\left(k_{m}+\ell_{r}\right) / 2} u_{m-1}\right|^{2}} \tau+\sum_{i=1}^{r}\left|\sqrt{\mathrm{c}_{i}^{(n+1)}} \mathscr{A}^{\left(\ell_{r}+\ell_{i}\right) / 2} u_{m-1}^{(n+1)}\right|^{2} \\
& \leq \frac{1}{\underline{\mathrm{c}}_{r}}\left|\mathscr{G}^{(n+1)}\left[\mathbf{u}^{(n+1)}\right]+f^{(n+1)}\right|^{2}+\frac{1}{4}\left|\sqrt{\mathrm{c}_{r}^{(n+1)}} \mathscr{A}^{\ell_{r}} u_{m-1}^{(n+1)}\right|^{2} \\
& +\frac{1}{2 \underline{\mathrm{c}}_{r}}\left|\left(\mathrm{~d}_{t}^{(n+1)} \underline{\mathrm{b}}_{\tau}\right)\right|_{L(\mathscr{H}, \mathscr{H})}\left|\sqrt{\mathrm{c}_{r}^{(n+1)}} \mathscr{A}^{\left(k_{m}+\ell_{r}\right) / 2} u_{m-1}^{(n+1)}\right|^{2}+\frac{1}{2}\left|\sqrt{\mathrm{b}^{(n+1)}} \mathscr{A}^{k_{m}} u_{m}^{(n+1)}\right|^{2} \\
& +\frac{1}{2 \underline{\mathrm{b}}}\left|\mathscr{A}^{\left(\ell_{r}-k_{m}\right) / 2}\left[\mathrm{~b}^{(n+1)} \mathscr{A}^{\left(k_{m}+\ell_{r}\right) / 2} u_{m-1}^{(n+1)}\right]-\left[\mathrm{b}^{(n+1)} \mathscr{A}^{\ell_{r}} u_{m-1}^{(n+1)}\right]\right|^{2} \\
& +\frac{1}{4}\left|\sqrt{c_{r}^{(n+1)}} \mathscr{A}^{\ell_{r}} u_{m-1}^{(n+1)}\right|^{2} \\
& +\frac{1}{\underline{c}_{r}}\left(\sum_{i=1}^{r}\left|\mathscr{A}^{\left(\ell_{i}-\ell_{r}\right) / 2}\left[\mathrm{c}_{i}^{(n+1)} \mathscr{A}^{\left(\ell_{r}+\ell_{i}\right) / 2} u_{m-1}^{(n+1)}\right]-\left[\mathrm{c}_{i}^{(n+1)} \mathscr{A}^{\ell_{i}} u_{m-1}^{(n+1)}\right]\right|\right)^{2} \text {. }
\end{aligned}
$$

The time integration step between (5.8) and (5.9), i.e., bewteen (5.13), (5.15) and the respective energy estimates, is replaced by $\tau$ weigthed summation, so that we get, e.g. in place of $\int_{0}^{t_{0}} \frac{d}{d t}\left|\mathscr{A}^{k_{m} / 2} u_{m}\right|^{2}(t) d t=\left|\mathscr{A}^{k_{m} / 2} u_{m}\left(t_{0}\right)\right|^{2}-\left|\mathscr{A}^{k_{m} / 2} u_{m}(0)\right|^{2}$ the identity

$$
\begin{aligned}
\tau \sum_{n=0}^{n_{0}-1} \mathrm{~d}_{t}^{(n+1)} \underline{\left|\mathscr{A}^{k_{m} / 2} u_{m}\right|^{2}} \tau & =\sum_{n=0}^{n_{0}-1}\left(\left|\mathscr{A}^{k_{m} / 2} u_{m}^{(n+1)}\right|^{2}-\left|\mathscr{A}^{k_{m} / 2} u_{m}^{(n)}\right|^{2}\right) \\
& =\left|\mathscr{A}^{k_{m} / 2} u_{m}^{\left(n_{0}\right)}\right|^{2}-\left|\mathscr{A}^{k_{m} / 2} u_{m}^{(0)}\right|^{2}
\end{aligned}
$$

Moreover, we use the following time discrete version of Gronwall's inequality:

$$
\begin{aligned}
& \eta^{(n)} \leq a^{(n)}+\tau \sum_{j=1}^{n} b^{(j)} \eta^{(j)} \quad \text { for all } n \in\{1, \ldots, N\} \\
& \Rightarrow \eta^{(n)} \leq a^{(n)}+\tau \sum_{j=1}^{n} a^{(j)} b^{(j)} \exp \left(\tau \sum_{i=j}^{n} b^{(i)}\right) \quad \text { for all } n \in\{1, \ldots, N\}
\end{aligned}
$$

for $\underline{\eta}_{\tau}, \underline{a}_{\tau}, \underline{b}_{\tau} \geq 0$, which follows by application of its continuous counterpart (5.19) to the piecewise constant interpolants of $\underline{\eta}_{\tau}, \underline{a}_{\tau}, \underline{b}_{\tau}$.

For the linear equation (6.3) we therefore get the following result.

Proposition 6.1. Under conditions (5.2), (5.4), (5.11), (5.21), with $c_{\mathrm{bc}}$ small enough, the time discretised initial value problem (6.3), (5.22) with the implicit Euler scheme (6.1) has a unique solution $\underline{\mathbf{u}}_{\tau} \in X_{\tau}$ and this solution satisfies the estimate

$$
\left\|\underline{\mathbf{u}}_{\tau}\right\|_{X_{\tau}} \leq C\left(\left\|\vec{u}^{0}\right\|_{X_{0}}+\|f\|_{L_{\tau}^{2}(\mathscr{H})}\right)
$$

with $C$ as in Proposition 5.1, in particular $C$ independent of $\tau$.

Proof. A Galerkin discretisation of (6.3), (5.22) with eigenfunctions $\phi_{i}$ of $\mathscr{A}$, i.e., an Ansatz $u_{j}^{(n)}(x)=\sum_{i=1}^{I} u_{i, j}^{(n)} \phi_{i}(x)$ after testing with $\phi_{k}, k \in\{1, \ldots, I\}$ yields $I$ linear $m \cdot n \times m \cdot n$ systems of

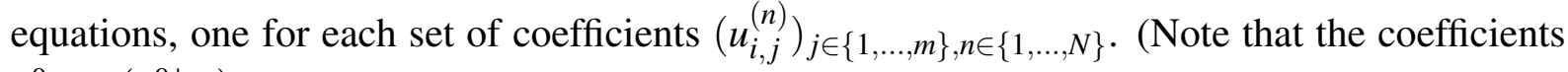
$u_{i, j}^{0}=\left(u_{j}^{0} \mid \phi_{i}\right)$ are fixed by the initial data.) Due to mutual orthogonality of the eigenfunctions, 
system $i_{1}$ is decoupled from system $i_{2}$ for $i_{1} \neq i_{2}$. System $i$ reads as

$$
\begin{aligned}
D_{t}^{(n+1)} \underline{\mathbf{u}}_{i} \tau & =\mathbf{A}^{(n+1)} \mathbf{u}_{i}^{(n+1)}+\left(0, \cdots, 0, \mathscr{G}^{(n+1)}\left[\mathbf{u}^{(n+1)}\right]+f_{i}^{(n)}\right)^{T}, \\
\text { with } \mathbf{A}^{(n)}= & \left(\begin{array}{ccccc}
0 & I & 0 & \cdots & 0 \\
0 & 0 & I & 0 & \cdots \\
\vdots & \ddots & \ddots & \ddots \\
0 & & -\sum_{v=1}^{r} \mathrm{c}_{v}\left(t_{n}\right) \lambda_{i}^{\ell_{v}} & -\mathrm{b}\left(t_{n}\right) \lambda_{i}^{k_{m}}
\end{array}\right),
\end{aligned}
$$

$f_{i}^{(n)}=\left(f^{(n)} \mid \phi_{i}\right), \mathbf{u}_{i}^{(n)}=\left(u_{i, 1}^{(n)}, \ldots, u_{i, m}^{(n)}\right)$ and $\mathbf{u}_{i}^{0}=\left(u_{i, 1}^{0}, \ldots, u_{i, m}^{0}\right)$, that is, the Galerkin discretisation simply replaces $\mathscr{A}$ by $\lambda_{i}$. Thus also the Galerkin approximation satisfies energy estimates analogous to those in Proposition 5.1, which implies uniqueness and, via Fredholm's alternative (since we are in finite dimensions now), also existence of a solution to (6.9). These energy estimates also yield uniform boundedness of the sequence $\left(\underline{\mathbf{u}}_{i}\right)_{i \in \mathbb{N}}$ defined by $\mathbf{u}^{(n)}(x)=$ $\sum_{i=1}^{I} \mathbf{u}_{i}^{(n)} \phi_{i}(x)$ in $X_{\tau}$ and therefore its weak convergence to a limit $\underline{\mathbf{u}}_{\tau}$ that by linearity can easily be verified to be a solution to (6.3), to which the energy estimates transfer as well. Uniqueness again follows from the energy estimates.

This allows to transfer the well-posedness result and energy estimates from Theorem 5.1 to the time discretised equation (6.2) under the following time discretised versions of the conditions (5.26)

$$
\begin{aligned}
& \left\|\underline{\mathbf{v}}_{\tau}\right\|_{X_{\tau}} \leq R \Rightarrow \\
& \left(\underline{\mathrm{b}(\mathbf{v})} \tau, \underline{\mathrm{c}_{i}(\mathbf{v})} \tau \in L_{\tau}^{\infty}(L(\mathscr{H}, \mathscr{H})), \quad \underline{\mathrm{d}_{t} \underline{\mathrm{b}(\mathbf{v})} \tau} \tau \underline{\underline{\mathrm{d}_{t} \underline{\mathrm{c}}_{i}(\mathbf{v})} \tau \tau} \tau \in L_{\tau}^{1}(L(\mathscr{H}, \mathscr{H}))\right) .
\end{aligned}
$$

Theorem 6.1. Under conditions (5.2), (6.10), (5.27), (5.28), there exists $R>0$ (sufficiently small) such that for any $\left\|\vec{u}_{0}\right\|_{X_{0}} \leq \frac{R}{C}$ the time discretised initial value problem (6.2), (5.22) with the Euler scheme (6.1) has a unique solution $\underline{\mathbf{u}}_{\tau} \in X_{\tau}$ and this solution satisfies the estimate

$$
\left\|\underline{\mathbf{u}}_{\tau}\right\|_{X_{\tau}} \leq C\left\|\vec{u}^{0}\right\|_{X_{0}}
$$

with C as in Proposition 5.1.

Verification of (6.10) for the models from Section 2 can be done by using the estimates (with $i=1, \kappa=\beta_{5}$ for BCBJK and $i=0, \kappa=\frac{2 \beta_{a}}{c^{2} \rho}$ for JMGT, Westervelt)

$$
\begin{aligned}
& \max _{n \in\{0, \ldots, N\}}\left\|1-\alpha\left(v_{i}^{(n)}\right)\right\|_{L(\mathscr{H}, \mathscr{H})}=\kappa\left\|\underline{v}_{\tau}\right\|_{L_{\tau}^{\infty}\left(L^{\infty}(\Omega)\right)} \\
& \tau \sum_{n=0}^{N}\left\|\frac{\alpha\left(v_{i}^{(n+1)}\right)-\alpha\left(v_{i}^{(n)}\right)}{\tau}\right\|_{L(\mathscr{H}, \mathscr{H})} \leq \kappa \tau \sum_{n=0}^{N}\left\|\mathrm{~d}_{t}^{(n+1)} \underline{v}_{\tau_{\tau}}\right\|_{L^{\infty}(\Omega)} \\
& \leq \kappa\left(\left\|\underline{v_{i+1}} \tau\right\|_{L_{\tau}^{1}\left(L^{\infty}(\Omega)\right)}+\kappa \tau \sum_{n=0}^{N}\left\|\mathrm{~d}_{t}^{(n+1)} \underline{v}_{\tau}-D_{t}^{(n+1)} \underline{v}_{\underline{v}_{\tau}}\right\|_{L^{\infty}(\Omega)}\right)
\end{aligned}
$$

due to $D_{t}^{(n+1)} \underline{v}_{i}=v_{i+1}^{(n+1)}$, and can therefore be estimated analogously to Section 5.3 in the Euler case $D_{t}=d_{t}$.

The time discretised versions of the models from Section 2 read as follows 
- BCBJK:

$$
\begin{aligned}
D_{t}^{(n+1)} \underline{\psi_{0}} \tau & =\psi_{1}^{(n+1)} \\
D_{t}^{(n+1)} \frac{\psi_{1}}{\tau} & =\psi_{2}^{(n+1)} \\
D_{t}^{(n+1)} \frac{\psi_{2}}{\tau} & =\frac{1}{1+\beta_{5} \psi_{1}^{(n+1)}}\left(\beta_{1} \Delta \psi_{2}^{(n+1)}-\beta_{2} \Delta^{2} \psi_{1}^{(n+1)}+\beta_{3} \Delta \psi_{1}^{(n+1)}-\beta_{4} \Delta^{2} \psi_{0}^{(n+1)}\right. \\
& \left.\quad-\beta_{5}\left(\psi_{2}^{(n+1)}\right)^{2}-2\left|\nabla \psi_{1}^{(n+1)}\right|^{2}-2 \nabla \psi_{0}^{(n+1)} \cdot \nabla \psi_{2}^{(n+1)}\right)
\end{aligned}
$$

- JMGT with $\beta_{0}=\frac{2 \beta_{a}}{\rho c^{2}}$ :

$$
\begin{aligned}
& D_{t}^{(n+1)} \underline{p_{0}} \tau=p_{1}^{(n+1)} \\
& D_{t}^{(n+1)} \underline{p_{1}} \tau=p_{2}^{(n+1)} \\
& D_{t}^{(n+1)} \underline{p}_{2}=\frac{1}{T_{\text {rel }}}\left(-\left(1-\beta_{0} p_{0}^{(n+1)}\right) p_{2}^{(n+1)}+b \Delta p_{1}^{(n+1)}+c^{2} \Delta p_{0}^{(n+1)}+\beta_{0}\left(p_{1}^{(n+1)}\right)^{2}\right)
\end{aligned}
$$

- Westervelt with $\beta_{0}=\frac{2 \beta_{a}}{\rho c^{2}}$ :

$$
\begin{aligned}
& D_{t}^{(n+1)} \underline{p}_{\tau}=p_{1}^{(n+1)} \\
& D_{t}^{(n+1)} \underline{p}_{\tau}=\frac{b}{1-\beta_{0} p_{0}^{(n+1)}} \Delta p_{1}^{(n+1)}+\frac{c^{2}}{1-\beta_{0} p_{0}^{(n+1)}} \Delta p_{0}^{(n+1)}+\frac{\beta_{0}}{1-\beta_{0} p_{0}^{(n+1)}}\left(p_{1}^{(n+1)}\right)^{2}
\end{aligned}
$$

Corollary 6.1. There exists $R>0$ (sufficiently small) such that for any $\left\|\vec{u}_{0}\right\|_{X_{0}} \leq \frac{R}{C}$ the semidiscrete PDEs (6.11), (6.12), (with $m=2$ ) (6.13) (with $m=1$ ), (5.22) with (6.1) have unique solutions $\underline{\mathbf{u}}_{\tau}=\left(\underline{u}_{0} \tau, \underline{u}_{\tau}, \ldots, \underline{u}_{m}\right) \in X_{\tau}$ and these solutions satisfy the estimate

$$
\left\|\left(\underline{u_{0}} \tau, \underline{u_{1}} \tau, \ldots, \underline{u_{m}} \tau\right)\right\|_{X_{\tau}} \leq C\left\|\vec{u}^{0}\right\|_{X_{0}}
$$

with

BCBJK: $X_{\tau}=L_{\tau}^{\infty}\left(H_{\diamond}^{4}(\Omega)\right) \cap L_{\tau}^{2}\left(H_{\diamond}^{4}(\Omega)\right) \cap H_{\tau}^{1}\left(H_{\diamond}^{4}(\Omega)\right)$

$$
\begin{aligned}
& \times L_{\tau}^{\infty}\left(H_{\diamond}^{3}(\Omega)\right) \cap L_{\tau}^{2}\left(H_{\diamond}^{4}(\Omega)\right) \cap H_{\tau}^{1}\left(H_{\diamond}^{2}(\Omega)\right) \times L_{\tau}^{\infty}\left(H_{0}^{1}(\Omega)\right) \cap L_{\tau}^{2}\left(H_{\diamond}^{2}(\Omega)\right), \\
X_{0}= & H_{\diamond}^{4}(\Omega) \times H_{\diamond}^{3}(\Omega) \times H_{0}^{1}(\Omega) .
\end{aligned}
$$

JMGT: $X_{\tau}=L_{\tau}^{\infty}\left(H_{0}^{1}(\Omega)\right) \cap L_{\tau}^{2}\left(H_{\diamond}^{2}(\Omega)\right) \cap H_{\tau}^{1}\left(H_{\diamond}^{2}(\Omega)\right)$

$$
\begin{aligned}
& \times L_{\tau}^{\infty}\left(H_{0}^{1}(\Omega)\right) \cap L_{\tau}^{2}\left(H_{\diamond}^{2}(\Omega)\right) \times L_{\tau}^{\infty}\left(L^{2}(\Omega)\right), \\
X_{0}= & H_{\diamond}^{2}(\Omega) \times H_{0}^{1}(\Omega) \times L^{2}(\Omega) .
\end{aligned}
$$

Westervelt: $X_{\tau}=L_{\tau}^{\infty}\left(H_{0}^{1}(\Omega)\right) \cap L_{\tau}^{2}\left(H_{\diamond}^{2}(\Omega)\right) \cap H_{\tau}^{1}\left(H_{\diamond}^{2}(\Omega)\right) \times L_{\tau}^{\infty}\left(H_{0}^{1}(\Omega)\right) \cap L_{\tau}^{2}\left(H_{\diamond}^{2}(\Omega)\right)$,

$$
X_{0}=H_{\diamond}^{2}(\Omega) \times H_{0}^{1}(\Omega) \text {. }
$$


6.2. Runge-Kutta methods. We consider a stiffly accurate Runge Kutta scheme with $s$ stages and Butcher tableau $\mathfrak{c} \mid$\begin{tabular}{c}
$\mathfrak{A}$ \\
\hline $\mathfrak{e}^{T} \mathfrak{A}$
\end{tabular} where $\mathfrak{A}=\left(\mathfrak{a}_{\mu \nu}\right)_{1 \leq \mu, v \leq s} \in \mathbb{R}^{s \times s}, \mathfrak{c}, \mathfrak{e}=(0, \ldots, 1)^{T} \in \mathbb{R}^{s}$, and we additionally assume

$$
\begin{gathered}
\sum_{v=1}^{s} a_{s v}=1, \quad a_{s v}>0, \quad \mathfrak{A} \text { regular }, \quad \mathfrak{B}:=\operatorname{diag}\left(a_{s}\right), \quad \mathfrak{C}:=\mathfrak{B A}^{-1} \\
\mathfrak{B A}+\mathfrak{A}^{T} \mathfrak{B}-a_{s} \cdot a_{s .}^{T}-\mathfrak{A}^{T} \mathfrak{C l 1}^{T} \mathfrak{C}^{T} \mathfrak{A} \text { positive semidefinite }
\end{gathered}
$$

cf. [1], which allows to conclude the following inequality cf. [1, Lemma 3.4]

$$
\left(x_{1}, \ldots, x_{s}\right) \mathfrak{C}\left(\begin{array}{c}
x_{1}-x_{0} \\
\vdots \\
x_{s}-x_{0}
\end{array}\right) \geq \frac{1}{2}\left(x_{s}^{2}-x_{0}^{2}\right)
$$

for all $x_{0}, x_{1}, \ldots x_{s} \in \mathbb{R}$. The latter carries over to the Hilbert space $\mathscr{H}$ in place of $\mathbb{R}$ in the sense that

$$
\sum_{\mu=1}^{s}\left(\sum_{v=1}^{s} \mathfrak{C}_{\mu v}\left(u^{v}-u^{0}\right) \mid u^{\mu}\right) \geq \frac{1}{2}\left(\left|u^{s}\right|^{2}-\left|u^{0}\right|^{2}\right)
$$

for all $u^{0}, u^{1}, \ldots u^{s} \in \mathscr{H}$. Note that (6.15) can be verfied under fairly general compatibility conditions, cf. [1, Theorem 3.1].

Inequality (6.16) is crucial for carrying over the energy estimates from Section 6.1 to the Runge Kutta discretisation of (5.3), (5.6), which according to [1, equations (1.1), (1.5), (4.1)], can be written as

$$
\sum_{v=1}^{s} \mathfrak{C}_{\mu \nu} \mathrm{d}_{t v}^{(n)} \underline{\mathbf{u}}_{\tau}+\mathbf{A}\left(\mathbf{U}_{\mu}^{(n)}\right) \mathbf{U}_{\mu}^{(n)}=\left(0, \cdots, 0, \mathscr{B}\left(\mathbf{U}_{\mu}^{(n)}\right)\left[\mathbf{U}_{\mu}^{(n)}\right]\right)^{T}
$$

and of (5.6)

$$
\left.\sum_{v=1}^{s} \mathfrak{C}_{\mu \nu} \mathrm{d}_{t \nu}^{(n)} \underline{\mathbf{u}}_{\tau}+\mathbf{A}_{\mu}^{(n)} \mathbf{U}_{\mu}^{(n)}=\left(0, \cdots, 0, \mathscr{G}_{\mu}^{(n)}\left[\mathbf{U}_{\mu}^{(n)}\right]+f_{\mu}^{(n)}\right)\right)^{T}
$$

respectively, where in both cases

$$
\mu=1, \ldots, s, \quad \mathbf{u}_{s}^{(n)}=: \mathbf{u}^{(n+1)}
$$

and we use the following abbreviations

$$
v_{v}^{(n)} \approx v\left(t_{n}+\mathfrak{c}_{v} \tau\right), v \in\left\{\mathbf{u}, u_{j}, \mathscr{G}, f\right\}, \quad \mathrm{d}_{t v}^{(n)} \underline{u}_{\tau}:=\frac{1}{\tau}\left(u_{v}^{(n)}-u^{(n)}\right),
$$

with the vector $\underline{u}_{\tau}=\left(u^{0,1}, \ldots, u^{0, s}, \ldots, u^{N, 1}, \ldots, u^{N, s}\right)$ of approximations at the time instances (including the stages beween them).

Analogously to the previous section, cf. (5.7), (5.14) we test with

$$
\left(0, \ldots, 0, \sum_{i=1}^{r} \mathscr{A}^{k_{m}}\left[\mathrm{c}_{i, \mu}^{(n)} \mathscr{A}^{\ell_{i}} u_{m-1, \mu}^{(n)}\right], \mathscr{A}^{k_{m}} u_{m, \mu}^{(n)}\right)^{T}
$$

and

$$
\left(0, \ldots, 0, \mathscr{A}^{k_{m}}\left[\mathrm{~b}_{\mu}^{(n)} \mathscr{A}^{\ell_{r}} u_{m-1, \mu}^{(n)}\right], \mathscr{A}^{\ell_{r}} u_{m-1, \mu}^{(n)}\right)^{T}
$$


respectively, sum up over $\mu$ and apply (6.16). The identity (6.6) is substituted by an estimate as follows.

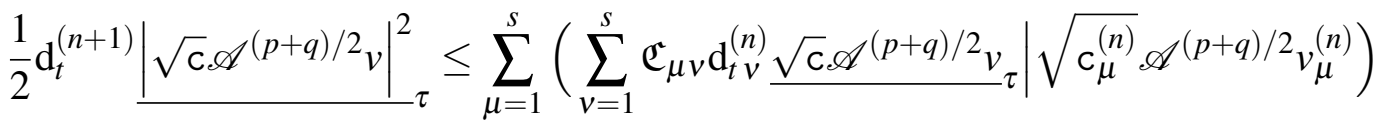

$$
\begin{aligned}
& =\sum_{\mu=1}^{s}\left(\sum_{v=1}^{s} \mathfrak{C}_{\mu \nu} \mathrm{d}_{t v}^{(n)} \underline{v}_{\tau} \mid \mathscr{A}^{p}\left[\mathrm{c}_{\mu}^{(n)} \mathscr{A}^{q} v_{\mu}^{(n)}\right]\right)+\sum_{\mu=1}^{s} \sum_{v=1}^{s} \mathfrak{C}_{\mu v} R_{\mu \nu}^{(n+1)},
\end{aligned}
$$

where we have used (6.16) in the first inequality and

$$
\begin{aligned}
& R_{\mu v}^{(n+1)}=\frac{1}{\tau}\left(\sqrt{\mathrm{c}_{v}^{(n)}} \mathscr{A}^{(p+q) / 2} v_{v}^{(n)}-\sqrt{\mathrm{c}^{(n)}} \mathscr{A}^{(p+q) / 2} v^{(n)} \mid \sqrt{\mathrm{c}_{\mu}^{(n)}} \mathscr{A}^{(p+q) / 2} v_{\mu}^{(n)}\right) \\
& -\frac{1}{\tau}\left(v_{v}^{(n)}-v^{(n)} \mid \mathscr{A}^{p}\left[\mathrm{c}_{\mu}^{(n)} \mathscr{A}^{q} v_{\mu}^{(n)}\right]\right) \\
& =\frac{1}{\tau}\left(\left(\sqrt{\mathrm{c}_{v}^{(n)}}-\sqrt{c^{(n)}}\right) \mathscr{A}^{(p+q) / 2} v_{v}^{(n)}+\sqrt{\mathrm{c}^{(n)}} \mathscr{A}^{(p+q) / 2}\left(v_{v}^{(n)}-v^{(n)}\right) \mid \sqrt{\mathrm{c}_{\mu}^{(n)}} \mathscr{A}^{(p+q) / 2} v_{\mu}^{(n)}\right) \\
& -\frac{1}{\tau}\left(v_{v}^{(n)}-v^{(n)} \mid \mathscr{A}^{p}\left[\mathrm{c}_{\mu}^{(n)} \mathscr{A}^{q} v_{\mu}^{(n)}\right]\right) \\
& =\frac{1}{\tau}\left(\left(\sqrt{\mathrm{c}_{v}^{(n)}}-\sqrt{c^{(n)}}\right) \mathscr{A}^{(p+q) / 2} v_{v}^{(n)} \mid \sqrt{\mathrm{c}_{\mu}^{(n)}} \mathscr{A}^{(p+q) / 2} v_{\mu}^{(n)}\right) \\
& +\frac{1}{\tau}\left(v_{v}^{(n)}-v^{(n)} \mid \mathscr{A}^{(p+q) / 2}\left[\sqrt{\mathrm{c}^{(n)}} \sqrt{\mathrm{c}_{\mu}^{(n)}} \mathscr{A}^{(p+q) / 2} v_{\mu}^{(n)}\right]-\mathscr{A}^{p}\left[\mathrm{c}_{\mu}^{(n)} \mathscr{A}^{q} v_{\mu}^{(n)}\right]\right) \\
& =\left(\left(\mathrm{d}_{t v}^{(n)} \underline{\sqrt{\mathrm{c}}}_{\tau}\right) \mathscr{A}^{(p+q) / 2} v_{v}^{(n)} \mid \sqrt{\mathrm{c}_{\mu}^{(n)}} \mathscr{A}^{(p+q) / 2} v_{\mu}^{(n)}\right) \\
& +\left(\mathrm{d}_{t v}^{(n)} \underline{v}_{\tau} \mid \mathscr{A}^{(p+q) / 2}\left[\mathrm{c}_{\mu}^{(n)} \mathscr{A}^{(p+q) / 2} v_{\mu}^{(n)}\right]-\mathscr{A}^{p}\left[\mathrm{c}_{\mu}^{(n)} \mathscr{A}^{q} v_{\mu}^{(n)}\right]\right) \\
& -\tau\left(\mathrm{d}_{t v}^{(n)} \underline{v}_{\tau} \mid \mathscr{A}^{(p+q) / 2}\left[\left(\mathrm{~d}_{t \mu}^{(n)} \underline{\sqrt{\mathrm{c}}}_{\tau}\right) \sqrt{\mathrm{c}_{\mu}^{(n)}} \mathscr{A}^{(p+q) / 2} v_{\mu}^{(n)}\right]\right) \text {. }
\end{aligned}
$$

Thus with $v=u_{m-1}$ and $\mathrm{c}=\mathrm{c}_{i}, p=k_{m}, q=\ell_{i}$ (likewise for $\mathrm{c}=\mathrm{b}, p=k_{m}, q=\ell_{r}$ as needed in the second energy estimate) we get, using $\sum_{v=1}^{s} \mathfrak{C}_{\mu \nu} \mathrm{d}_{t v}^{(n)} \underline{u}_{\tau, m-1}=u_{m}^{(n+1)}$, that

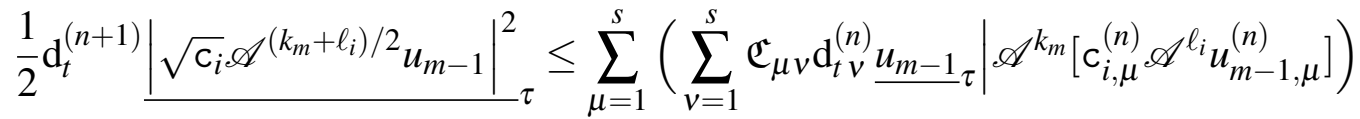

$$
\begin{aligned}
& +\sum_{\mu=1}^{s} \sum_{v=1}^{s} \mathfrak{c}_{\mu v}\left(\left(\mathrm{~d}_{t v}^{(n)} \underline{\sqrt{\mathrm{c}_{i}}} \tau\right) \mathscr{A}^{\left(k_{m}+\ell_{i}\right) / 2} u_{m-1, v}^{(n)} \mid \sqrt{\mathrm{c}_{\mu}^{(n)}} \mathscr{A}^{\left(k_{m}+\ell_{i}\right) / 2} u_{m-1, \mu}^{(n)}\right) \\
& +\sum_{\mu=1}^{s}\left(\mathscr{A}^{k_{m}} u_{m}^{(n+1)} \mid \mathscr{A}^{\left(\ell_{i}-k_{m}\right) / 2}\left[\mathrm{c}_{\mu}^{(n)} \mathscr{A}^{\left(k_{m}+\ell_{i}\right) / 2} u_{m-1, \mu}^{(n)}\right]-\left[\mathrm{c}_{\mu}^{(n)} \mathscr{A}^{\ell_{i}} u_{m-1, \mu}^{(n)}\right]\right) \\
& -\tau \sum_{\mu=1}^{s}\left(\mathscr{A}^{k_{m}} u_{m}^{(n+1)} \mid \mathscr{A}^{\left(\ell_{i}-k_{m}\right) / 2}\left[\left(\mathrm{~d}_{t \mu}^{(n)} \underline{\sqrt{\mathrm{c}}} \tau\right) \sqrt{\mathrm{c}_{\mu}^{(n)}} \mathscr{A}^{\left(k_{m}+\ell_{i}\right) / 2} u_{m-1, \mu}^{(n)}\right]\right) \text {. }
\end{aligned}
$$

Altogether we therefore obtain the same results as for the implicit Euler method.

Theorem 6.2. Under conditions (5.2), (6.10), (5.27), (5.28), there exists $R>0$ (sufficiently small) such that for any $\left\|\vec{u}_{0}\right\|_{X_{0}} \leq \frac{R}{C}$ the time discretised initial value problem (6.17), (5.22) 
with a stiffly accurate Runge Kutta method satisfying (6.15) has a unique solution $\underline{\mathbf{u}}_{\tau} \in X_{\tau}$ and this solution satisfies the estimate

$$
\left\|\underline{\mathbf{u}}_{\tau}\right\|_{X_{\tau} \leq C\left\|\vec{u}^{0}\right\|_{X_{0}}}
$$

with C as in Proposition 5.1.

Applying this to the models from Section 2 we get

- BCBJK:

$$
\begin{aligned}
& \sum_{v=1}^{s} \mathfrak{C}_{\mu \nu} \mathrm{d}_{t v}^{(n)} \underline{\psi_{0}} \tau=\psi_{1, \mu}^{(n)}, \\
& \sum_{v=1}^{s} \mathfrak{C}_{\mu \nu} \mathrm{d}_{t v}^{(n)} \underline{\psi_{1}} \tau=\psi_{2, \mu}^{(n)}, \\
& \sum_{v=1}^{s} \mathfrak{C}_{\mu \nu} \mathrm{d}_{t v}^{(n)} \underline{\psi_{2}} \tau=\frac{1}{1+\beta_{5} \psi_{1, \mu}^{(n)}}\left(\beta_{1} \Delta \psi_{2, \mu}^{(n)}-\beta_{2} \Delta^{2} \psi_{1, \mu}^{(n)}+\beta_{3} \Delta \psi_{1, \mu}^{(n)}-\beta_{4} \Delta^{2} \psi_{0, \mu}^{(n)}\right. \\
&\left.-\beta_{5}\left(\psi_{2, \mu}^{(n)}\right)^{2}-2\left|\nabla \psi_{1, \mu}^{(n)}\right|^{2}-2 \nabla \psi_{0, \mu}^{(n)} \cdot \nabla \psi_{2, \mu}^{(n)}\right) .
\end{aligned}
$$

- JMGT with $\beta_{0}=\frac{2 \beta_{a}}{\rho c^{2}}$ :

$$
\begin{aligned}
& \sum_{v=1}^{s} \mathfrak{C}_{\mu v} \mathrm{~d}_{t v}^{(n)} \underline{p_{0}} \tau=p_{1, \mu}^{(n)}, \\
& \sum_{v=1}^{s} \mathfrak{C}_{\mu v} \mathrm{~d}_{t v}^{(n)}{\underline{p_{1}} \tau}_{\tau}=p_{2, \mu}^{(n)}, \\
& \sum_{v=1}^{s} \mathfrak{C}_{\mu v} \mathrm{~d}_{t v}^{(n)} \underline{p}_{\tau}=\frac{1}{T_{r e l}}\left(-\left(1-\beta_{0} p_{0, \mu}^{(n)}\right) p_{2, \mu}^{(n)}+b \Delta p_{1, \mu}^{(n)}+c^{2} \Delta p_{0, \mu}^{(n)}+\beta_{0}\left(p_{1, \mu}^{(n)}\right)^{2}\right) .
\end{aligned}
$$

- Westervelt with $\beta_{0}=\frac{2 \beta_{a}}{\rho c^{2}}$ :

$$
\begin{aligned}
& \sum_{v=1}^{s} \mathfrak{C}_{\mu \nu} \mathrm{d}_{t v}^{(n)} \underline{p}_{0}=p_{1, \mu}^{(n)}, \\
& \sum_{v=1}^{s} \mathfrak{C}_{\mu \nu} \mathrm{d}_{t v}^{(n)}{\underline{p_{1}} \tau}_{\tau}=\frac{b}{1-\beta_{0} p_{0, \mu}^{(n)}} \Delta p_{1, \mu}^{(n)}+\frac{c^{2}}{1-\beta_{0} p_{0, \mu}^{(n)}} \Delta p_{0, \mu}^{(n)}+\frac{\beta_{0}}{1-\beta_{0} p_{0, \mu}^{(n)}}\left(p_{1, \mu}^{(n)}\right)^{2} .
\end{aligned}
$$

Corollary 6.2 (Existence of time-discrete solutions). There exists $R>0$ (sufficiently small but independent of $\tau$ ) such that for any $\left\|\vec{u}_{0}\right\|_{X_{0}} \leq \frac{R}{C}$ the semidiscrete PDEs (6.11), (6.12), (6.13) with initial data (5.22) and a stiffly accurate Runge Kutta method satisfying (6.15) have unique solutions $\underline{\mathbf{u}}_{\tau}=\left(\underline{u}_{0}, \underline{u}_{\tau}, \ldots, \underline{u}_{m}\right) \in X_{\tau}$ and these solutions satisfy the estimate

$$
\left\|\left(\underline{u}_{0} \tau, \underline{u_{1}} \tau, \ldots, \underline{u_{m}} \tau\right)\right\|_{X_{\tau}} \leq C\left\|\vec{u}^{0}\right\|_{X_{0}}
$$

with $X_{\tau}$ as in (6.14).

For the following convergence result we will consider the piecewise linear interpolants

$$
v^{\tau}(t)=v_{v}^{(n)}+\frac{v_{v+1}^{(n)}-v_{v}^{(n)}}{\left(\mathfrak{c}_{v+1}-\mathfrak{c}_{v}\right) \tau}\left(t-t_{n}-\mathfrak{c}_{v} \tau\right), \quad t \in\left[t_{n}+\mathfrak{c}_{v} \tau, t_{n}+\mathfrak{c}_{v+1} \tau\right)
$$


of grid functions $\underline{v}_{\tau}$.

Corollary 6.3 (Convergence). Let $R$ be chosen as in Corollary 6.2, assume $\left\|\vec{u}_{0}\right\|_{X_{0}} \leq \frac{R}{C}$, and denote by $\mathbf{u}^{\tau}$ the piecewise linear interpolant of the solution $\underline{\mathbf{u}}_{\tau}=\left(\underline{u}_{0} \tau, \underline{u}_{1}, \ldots, \underline{u}_{m_{\tau}}\right) \in X_{\tau}$ of one of the semidiscrete first order systems (6.11), (6.12), (6.13), with initial data (5.22) and a stiffly accurate Runge Kutta method satisfying (6.15). Then the family of these interpolants $\left(\mathbf{u}^{\tau}\right)_{\tau \in(0, \bar{\tau})}$ converges weakly* in $X$ to the solution of the first order reformulation of the respective nonlinear initial value problem (2.3), (2.2), (2.1), with initial data (5.22) as $\tau$ tends to zero, that is,

$$
\mathbf{u}^{\tau} \stackrel{*}{\rightarrow} \mathbf{u} \text { in } X \text { as } \tau \rightarrow 0 \text {. }
$$

In particular, $\mathbf{u}^{\tau}$ converges strongly to $\mathbf{u}$ in any space that is compactly embedded into $X$.

Proof. According to Corollary 6.2, the family of interpolants $\left(\mathbf{u}^{\tau}\right)_{\tau \in(0, \bar{\tau})}$ is uniformly bounded in $X$ as defined in (5.17). Therefore, there exist a weakly* convergent subsequence, which we denote by $\left(\mathbf{u}^{k}\right)_{k \in \mathbb{N}}$.

The fact that any weakly* convergent subsequence $\left(\mathbf{u}^{k}\right)_{k \in \mathbb{N}}$ tends to the unique solution $\mathbf{u}$ can be seen by considering, for arbitrary $\phi \in C_{0}^{\infty}(\Omega), \theta \in C_{0}^{\infty}(0, T)$ and $j \in\{0, \ldots, m\}$ the integrals

$$
L_{j}^{k}+N_{j}^{k}:=\int_{0}^{T} \int_{\Omega}\left(\left(\mathscr{L}_{j}\left(\mathbf{u}^{k}-\mathbf{u}\right)(x, t)+\left(\mathscr{N}_{j}\left(\mathbf{u}^{k}\right)-\mathscr{N}_{j}(\mathbf{u})\right)(x, t)\right) \phi(x) \theta(t) \mathrm{d} x \mathrm{~d} t,\right.
$$

where $\mathscr{L}_{j}$ and $\mathscr{N}_{j}$ are the linear and nonlinear parts of the differential operators acting on the respective component (note that in case of BCBJK the linear part also contains a term in $\mathscr{B}$ ). It is straigthforward to see that $L_{j}^{k} \rightarrow 0$ as $k \rightarrow \infty$ due to the weak convergence $\mathbf{u}^{k} \stackrel{*}{\rightarrow} \mathbf{u}$ in $X$. For the nonlinear parts $N_{j}^{k}$ we note that they vanish for $j \in\{0, \ldots, m-1\}$ and that $N_{m}^{k}$ must be considered for the particular PDEs separately. We do so explicitely only for the Westervelt equation, where we have

$$
\mathscr{N}_{m}\left(\mathbf{u}^{k}\right)-\mathscr{N}_{j}(\mathbf{u})=\beta_{0}\left(\frac{1}{1-\beta_{0} u_{0}^{k}}\left(u_{1}^{k}\right)^{2}-\frac{1}{1-\beta_{0} u_{0}}\left(u_{1}\right)^{2}\right) .
$$

Hence with the functions $\omega^{k}=\frac{\beta_{0}^{2}\left(u_{1}^{k}\right)^{2}}{\left(1-\beta_{0} u_{0}^{k}\right)\left(1-\beta_{0} u_{0}\right)}+\frac{\beta_{0}\left(u_{1}^{k}+u_{1}\right)}{\left(1-\beta_{0} u_{0}\right)}$ that are uniformly (with respect to $k$ ) bounded in $L^{\infty}\left(0, T ; L^{2}(\Omega)\right)$ due to nondegeneracy and boundedness of both the continuous and the discrete solution, we have

$$
\begin{aligned}
N_{m}^{k} & =\int_{0}^{T} \int_{\Omega}\left(\omega^{k}(x, t)\left(u_{0}^{k}(x, t)-u_{0}(x, t)\right) \phi(x) \theta(t) \mathrm{d} x \mathrm{~d} t\right. \\
& \leq\left\|\omega^{k}\right\|_{L^{\infty}\left(0, T ; L^{2}(\Omega)\right.}\left\|u_{0}^{k}-u_{0}\right\|_{L^{2}\left(0, T ; L^{2}(\Omega)\right.}\|\phi\|_{L^{\infty}(\Omega)}\|\theta\|_{L^{2}(0, T)} \rightarrow 0 \text { as } k \rightarrow \infty
\end{aligned}
$$

due to compactness of the embedding $H^{1}\left(0, T ; H_{\diamond}^{2}(\Omega)\right) \rightarrow L^{2}\left(0, T, L^{2}(\Omega)\right)$. Similarly in principle, but with more involved computations, convergence to zero of the nonlinear terms can also be shown for JMGT and BCBJK. We point to $[17,21]$ and the fact that the same differences of nonlinear terms have to be tackled when studying convergence as one of the physical parameters in the PDE tends to zero. These estimates can be directly used here, since we have the same or higher regularity for BCBJK and JMGT, see Remark 5.2.

A subsequence-subsequence argument together with uniqueness of solutions to the respective limiting (time continuous) equations yields the assertion. 


\section{REFERENCES}

[1] E. Emmrich, M. Thalhammer, Stiffly accurate Runge-Kutta methods for nonlinear evolution problems governed by a monotone operator, Math. Comput. 79 (2010), 785-806.

[2] J. Gwinner, M. Thalhammer, Full discretisations for nonlinear evolutionary inequalities based on stiffly accurate Runge—Kutta and hp-finite element methods, Found. Comput. Math. 14 (2014), 913-949.

[3] O. V. Abramov, High-Intensity Ultrasonics: Theory and Industrial Applications, CRC Press 10, 1999.

[4] T. Dreyer, W. Krauss, E. Bauer, R. E. Riedlinger, Investigations of compact self focusing transducers using stacked piezoelectric elements for strong sound pulses in therapy, Ultrasonics Symposium IEEE 2 (2000), $1239-1242$

[5] M. Kaltenbacher, H. Landes, J. Hoffelner, R. Simkovics, Use of modern simulation for industrial applications of high power ultrasonics, Ultrasonics Symposium IEEE 1 (2002), 673-678.

[6] D. G. Crighton, Model equations of nonlinear acoustics, Ann. Rev. Fluid Mech. 11 (1979), 11-33.

[7] B. O. Enflo, C. M. Hedberg, Theory of Nonlinear Acoustics in Fluids, Springer Science \& Business Media 67, 2006.

[8] M. F. Hamilton, D. T. Blackstock, Nonlinear Acoustics, Academic press San Diego 1, 1998.

[9] V. P. Kuznetsov, Equations of nonlinear acoustics, Sov. Phys. Acoust. 16 (1971), 467-470.

[10] M. B. Lesser, R. Seebass, The structure of a weak shock wave undergoing reflexion from a wall, J. Fluid Mech. 31 (1968), 501-528.

[11] S. Makarov, M. A. Ochmann, Nonlinear and thermoviscous phenomena in acoustics, part I, Acustica, Acta Acustica 82 (1996), 579-606.

[12] S. Makarov, M. A. Ochmann, Nonlinear and thermoviscous phenomena in acoustics, part II, Acustica, Acta Acustica 83 (1997), 197-222.

[13] S. Makarov, M. A. Ochmann, Nonlinear and thermoviscous phenomena in acoustics, part III, Acustica, Acta Acustica 83 (1997), 827-846.

[14] A. Pierce, An Introduction to Its Physical Principles and Applications, Acoustical Society of America, 1989.

[15] P. J. Westervelt, Parametric acoustic array, J. Acoustical Soc. Amer. 35 (1963), 535-537.

[16] B. Kaltenbacher, Mathematics of nonlinear acoustics, Evol. Equ. Control Theory 4 (2015), 447-491.

[17] B. Kaltenbacher, M. Thalhammer, Fundamental models in nonlinear acoustics part I. Analytical comparison, Math. Models Methods Appl. Sci. 28 (2018), 2403-2455.

[18] B. Kaltenbacher, I. Lasiecka, Global existence and exponential decay rates for the Westervelt equation, Discrete Contin. Dyn. Syst. 2 (2009), 503-523.

[19] S. Meyer, M. Wilke, Optimal regularity and long-time behavior of solutions for the Westervelt equation, Appl. Math. Optim. 64 (2011), 257-271.

[20] P. M. Jordan, Second-sound phenomena in inviscid, thermally relaxing gases, Discrete Contin. Dyn. Syst. 19 (2014), 2189-2205.

[21] B. Kaltenbacher, V. Nikolić, On the Jordan-Moore-Gibson-Thompson equation: well-posedness with quadratic gradient nonlinearity and singular limit for vanishing relaxation time, Math. Models Methods Appl. Sci. 29 (2019), 2523-2556.

[22] B. Kaltenbacher, I. Lasiecka, R. Marchand, Wellposedness and exponential decay rates for the MooreGibson-Thompson equation arising in high intensity ultrasound, Control Cybernet. 40 (2011), 971-988.

[23] B. Kaltenbacher, I. Lasiecka, M. K. Pospieszalska, Well-posedness and exponential decay of the energy in the nonlinear Jordan-Moore-Gibson-Thompson equation arising in high intensity ultrasound, Math. Models Methods Appl. Sci. 22 (2012), 1250035.

[24] I. Lasiecka, X. Wang, Moore-Gibson-Thompson equation with memory, part II: general decay of energy, J. Differential Equations 259 (2015), 7610-7635.

[25] I. Lasiecka, X. Wang, Moore-Gibson-Thompson equation with memory, part I: exponential decay of energy, Z. Angew. Math. Phys. 67 (2016), 17.

[26] S. Liu, R. Triggiani, An inverse problem for a third order PDE arising in high-intensity ultrasound: Global uniqueness and stability by one boundary measurement, J. Inverse Ill-Posed Probl. 21 (2013), 825-869. 
[27] R. Marchand, T. McDevitt, R. Triggiani, An abstract semigroup approach to the third-order Moore-GibsonThompson partial differential equation arising in high-intensity ultrasound: structural decomposition, spectral analysis, exponential stability, Math. Methods Appl. Sci. 35 (2012), 1896-1929.

[28] D. T. Blackstock, Approximate equations governing finite-amplitude sound in thermoviscous fluids, Technical Report GD/E Report GD-1463-52 (1963), 1463.

[29] R. Brunnhuber, P. M. Jordan, On the reduction of Blackstock's model of thermoviscous compressible flow via Becker's assumption, Int. J. Non-Linear Mech. 78 (2016), 131-132.

[30] B. Kaltenbacher, Well-posedness of a general higher order model in nonlinear acoustics, Appl. Math. Lett. 63 (2017), 21-27.

[31] W. Dörfler, H. Gerner, R. Schnaubelt, Local well-posedness of a quasilinear wave equation, Appl. Anal. 95 (2016), 2110-2123.

[32] B. Kaltenbacher, V. Nikolić, Parabolic approximation of quasilinear wave equations with applications in nonlinear acoustics, arXiv preprint arXiv:2011.07360, 2020.

[33] L. C. Evans, Partial Differential Equations, Graduate Studies in Mathematics 19. AMS, Providence, RI, 1998.

[34] G. Teschl, Ordinary Differential Equations and Dynamical Systems, American Mathematical Society, 2012.

[35] E. Emmrich, Gewöhnliche und Operator-Differentialgleichungen: Eine integrierte Einführung in Randwertprobleme und Evolutionsgleichungen für Studierende, Vieweg-Studium: Mathematik, 2004. 